\$5. Eastern Parifia.

$54740-4743$

Fobiy $7^{\pi}$. to Feby $24^{\pi}$ ilgos. 
115: 1904-5 
$\begin{aligned} \text { xext a. }- & 4741 \\ & 4744\end{aligned}$

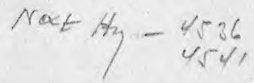




$$
\begin{aligned}
& \text { 3.1.416) } \\
& .0000 .3183 \\
& \frac{94248}{57520} \\
& 09 \\
& 3 \frac{1 \times 16}{61040} \\
& \frac{251318}{9.7 .12}
\end{aligned}
$$

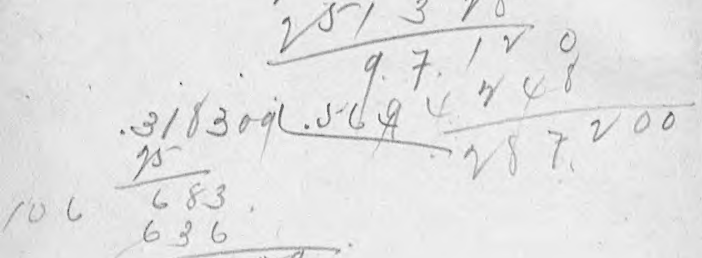

$$
\begin{aligned}
& 118 \frac{636}{4.7996} \\
& 1.128
\end{aligned}
$$


$5070 \times 9$

PL. 86.

$\nabla^{2} \quad 4833$.

No. 44 y 402

Lucas

Machine.

Date 7 , Fetr 1905

Reel. Liecao
Turns
Cor.t
Depth 2319 fath.

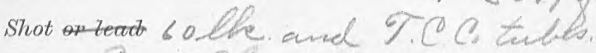

Bottom De.

Bottom temperature $36 .-2$

No. of thermometer 108023

Cor.

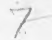

Corrected temperature
Air $88^{\circ}$
Surface

$3 \times 5$

Trawt or dredge

\begin{tabular}{|c|c|c|c|c|}
\hline \multicolumn{2}{|c|}{ SOUNDING WIRE. } & \multirow{2}{*}{ TURNS. } & \multicolumn{2}{|c|}{ DREDGE ROPE. } \\
\hline Dows. Al & DT UP. & & DOwN. & UP, \\
\hline 2.0018 & $8-53-20$ & 0 & & \\
\hline $2 \cdot 50$ & $3-2-20$ & 100 & & \\
\hline $3=50$ & $51-30$ & 200 & & \\
\hline $4-4 / 5$ & $50-40$ & 300 & & \\
\hline $5-35$ & $49-52$ & 400 & 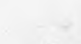 & \\
\hline $6-25$ & $48-30$ & 500 & ' & \\
\hline $3-15$ & $47-67$ & 600 & & \\
\hline $8-10$ & $46-52$ & 700 & & \\
\hline 9.05 & $45^{2}-50$ & 800 & & \\
\hline $10-05$ & $44-5 y$ & 900 & & \\
\hline $11=0.5$ & $4,3-52$ & 1000 & & \\
\hline $12 \cdot 05$ & $42 \cdot 70$ & 1100 & & \\
\hline $13-03$ & 41.40 & 1200 & & \\
\hline $14-05$ & 40.40 & 1300 & & -5 \\
\hline $15^{2}-0.5$ & $39-10$ & 1400 & & \\
\hline $16-06$ & $38-00$ & 1500 & 3 & \\
\hline $17-0.5$ & $36-55$ & 1600 & & \\
\hline $18-10$ & $35=35$ & 1700 & & \\
\hline $19-15$ & $34-20$ & 1800 & & \\
\hline $20-20$ & $35-0$ & 1900 & & $5-$ \\
\hline $21-30$ & 3). -43 & 2000 & & \\
\hline $22-40$ & $30-20$ & 2100 & & - \\
\hline $23-50$ & 29.00 & 2200 & & \\
\hline $\begin{array}{ll}25: & 00 \\
25-10\end{array} \mid$ & $\mid \begin{array}{r}27-25 \\
8-27-10\end{array}$ & $\begin{array}{l}2300 \\
2319 \\
4400\end{array}$ & & \\
\hline
\end{tabular}


Lat $18^{\circ} 29.4^{\prime} 5$.

$20 n 8.130^{\circ} \sqrt{0} .8$

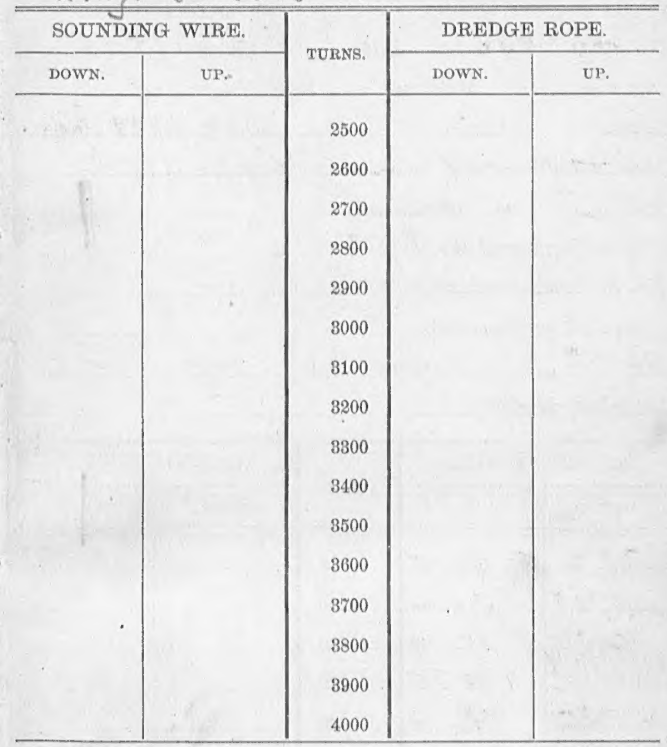

SERIAL TEMPERATURES

\begin{tabular}{r|r|r|r|r}
\hline \hline DEPTH. & TEMP. & NO. OF THER. & COR. & COR. TEMPP. \\
\hline 25 & & & & \\
\hline 50 & & & & \\
100 & & & \\
200 & & & \\
300 & & & \\
400 & & & \\
500 & & & \\
600 & & & \\
700 & & & \\
800 & & & \\
900 & & & \\
1000 & & & & \\
\hline
\end{tabular}

REMARKS: 


\section{$0.1 .=69.9$}

4834,

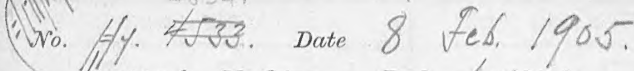
Wucas Machine. Reel. \ucás
Turns
Corit
Depth 2/94 כives.

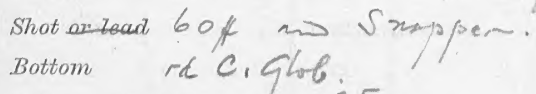

Bottom temperature $\sqrt{5.2}$

No. of thermometer 108023. Cor. 7

Corrected temperature

Air $83^{\circ}$ Surface $82^{\circ}$ Drift

Trazbor dxedge

\begin{tabular}{|c|c|c|c|c|}
\hline \multicolumn{2}{|c|}{ SOUNDING WIRE. } & \multirow{2}{*}{ TURNS. } & \multicolumn{2}{|c|}{ DREDGE ROPE. } \\
\hline$a m^{\text {DOWN. }}$ & UP. & & Dows. & ur. \\
\hline $8: 03.00$ & $8: 5 \% \cdot \sqrt{3}$ & 0 & & \\
\hline $03, \sqrt{5}$ & 51.00 & 100 & & \\
\hline OH. 4/5 & 50.10 & 200 & & \\
\hline a.5.25 & 49.15 & 300 & & \\
\hline 06.30 & 48.20 & 400 & & \\
\hline 07.30 & 47.25 & 500 & & \\
\hline 08.30 & 46.30 & 600 & & \\
\hline 09.30 & $45: 30$ & 700 & & \\
\hline 10.25 & 44.30 & 800 & & \\
\hline 11.20 & $4 / 3.30$ & 900 & & \\
\hline 12.20 & 42.25 & 1000 & & \\
\hline 19.25 & 41.20 & 1100 & & \\
\hline 14.25 & 40.15 & 1200 & & \\
\hline 15.30 & 29.10 & 1800 & & \\
\hline 16.30 & 37.55 & 1400 & & \\
\hline 17.35 & 36.45 & 1500 & & \\
\hline 18.40 & 45.10 & 1600 & & \\
\hline $19.1 / 5$ & 14.15 & 1700 & & \\
\hline $20 . \sqrt{2}$ & 32.12 & 1800 & & \\
\hline $21 . \sqrt{5}$ & 31.40 & 1900 & & \\
\hline $2 \leqslant .00$ & 30.15 & 2000 & & \\
\hline 24.10 & 28.50 & 2100 & & \\
\hline $8: 25.15$ & 8.27 .15 & -9200 & & \\
\hline & & $\begin{array}{l}2300 \\
2400\end{array}$ & & \\
\hline
\end{tabular}


No. Hy rasy Date QFebry.05 Licas Machine. Reel. Sicas

Turns

$$
\text { Cor }+
$$

Depth 2185frizs.

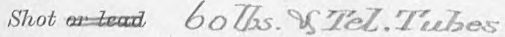

Bottom,

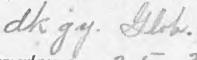

Bottom temperature 3.5 .3

No. of thermometer 108023

Cor.

Corrected temperature

Air $82^{\circ}$ Surface $52^{\circ}$ Drift

Trawl or dredge

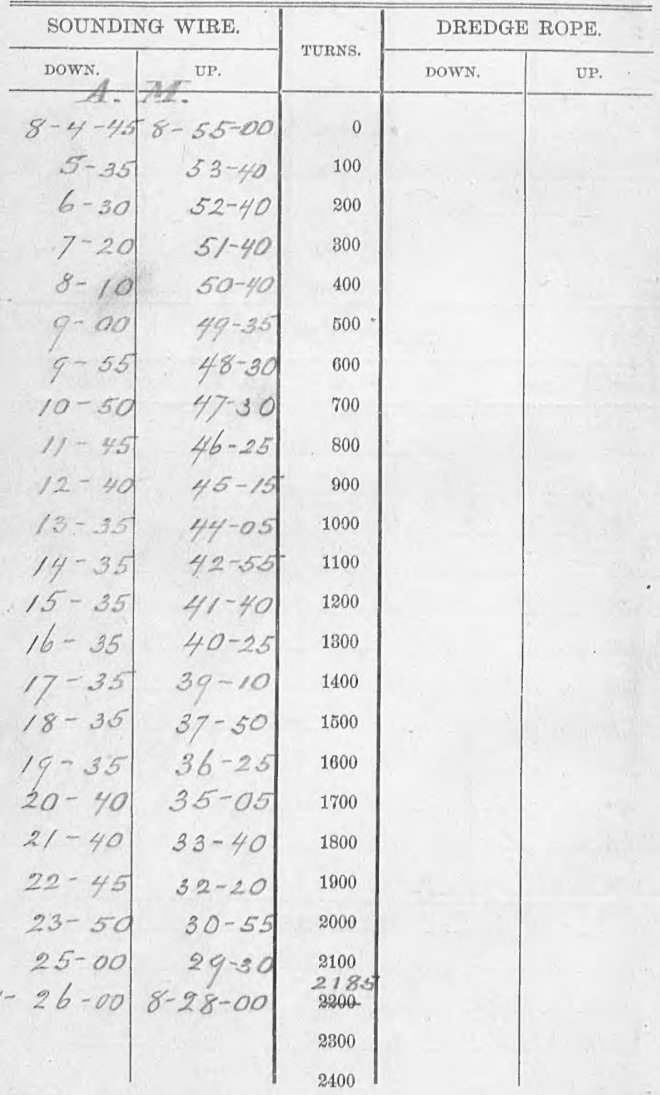


Ieat. $13^{\circ} 51: 0^{\prime \prime} 5$

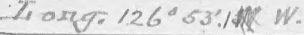

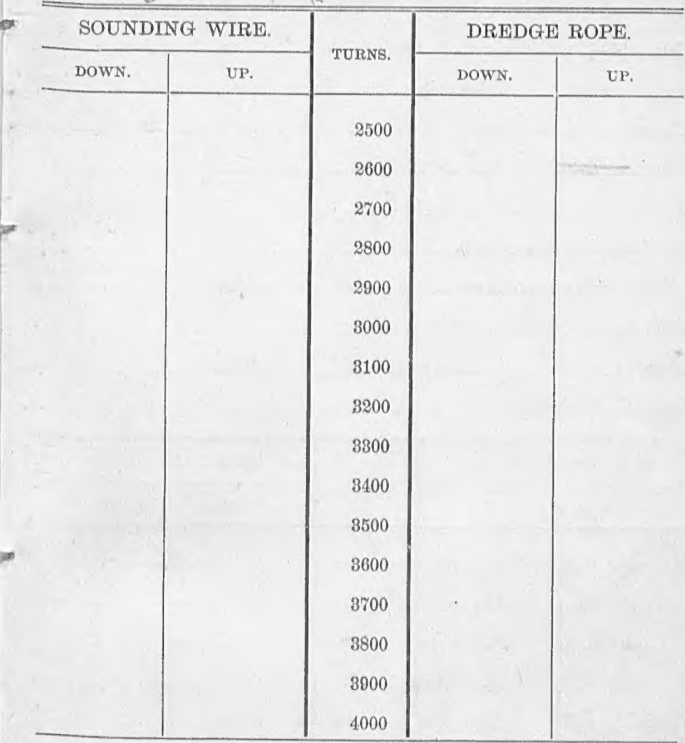

SERIAL TEMPERATURES

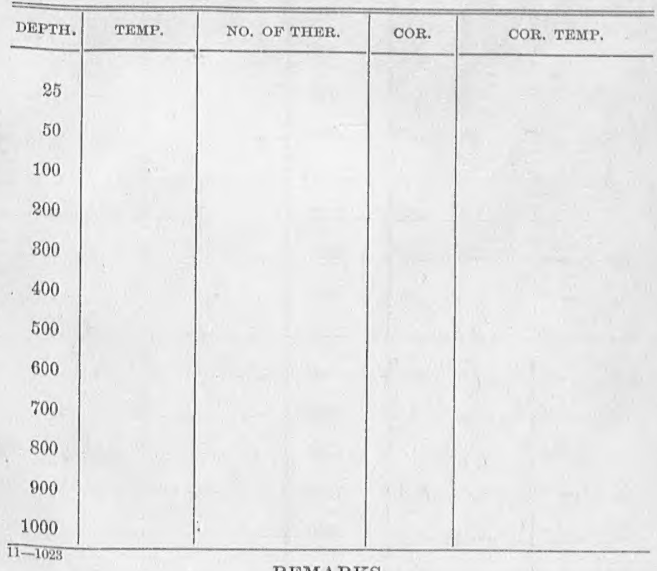

REMARKS: 
No. $4 \%-3=34$.

Date 10 Fisteos Machine. Reel. Tureaces

Cor. +

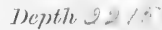

\section{Turus}

Shot ar teat 6 olbs $\times$ 9. Terber

Bottom Lify.y. Whote Oz.

Bottom temperature is:a.

No. of thermometer 108023

Cor.

Corrected temperature

Air $82^{\circ} \quad$ Surface $80^{\circ}$ Drift

Trawt or dredge

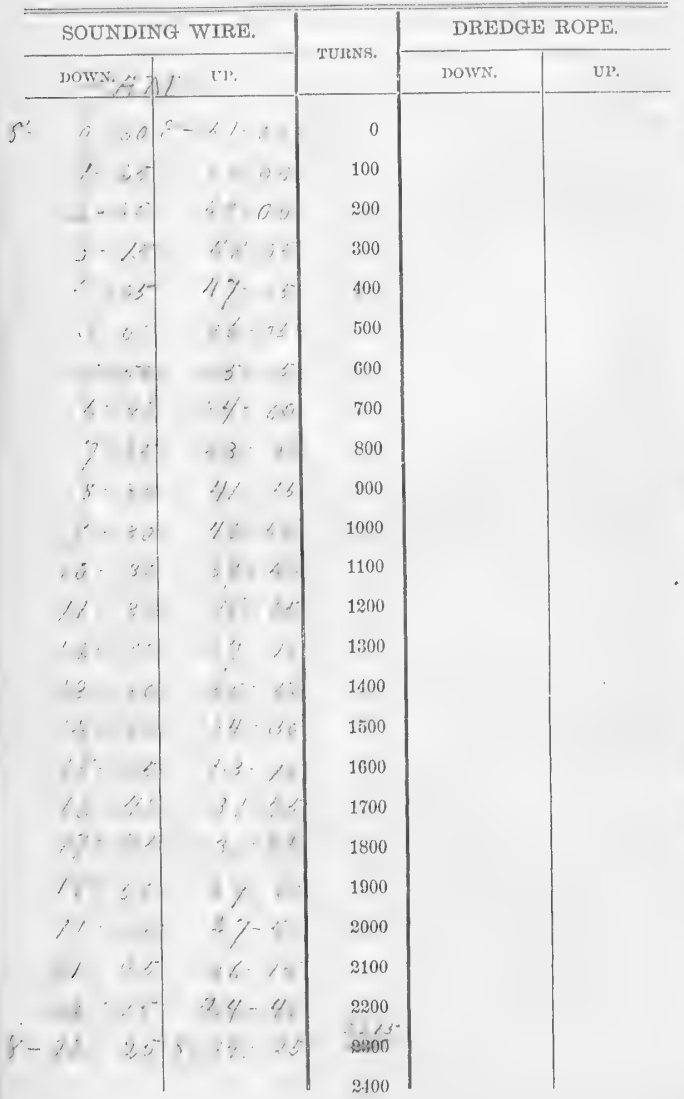




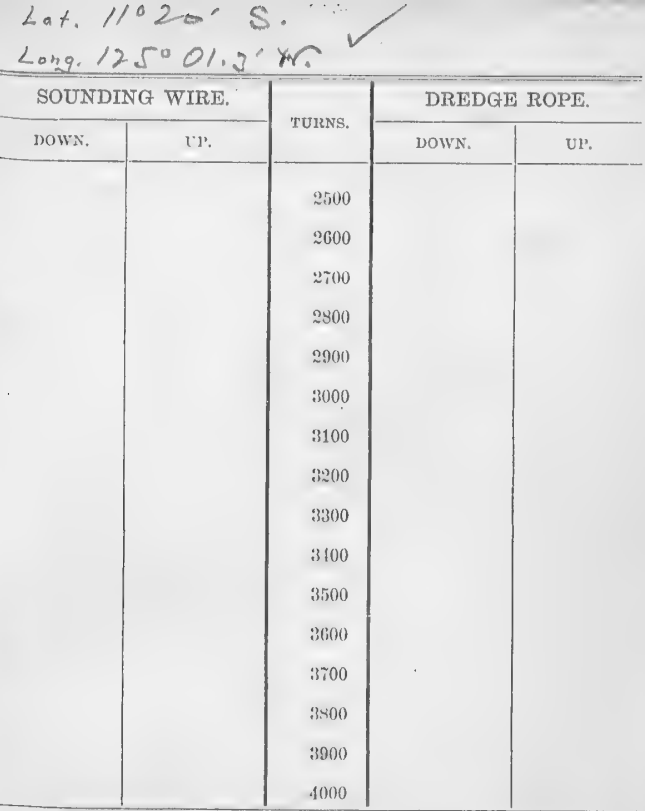

SEIRLAL TEALPERATURES

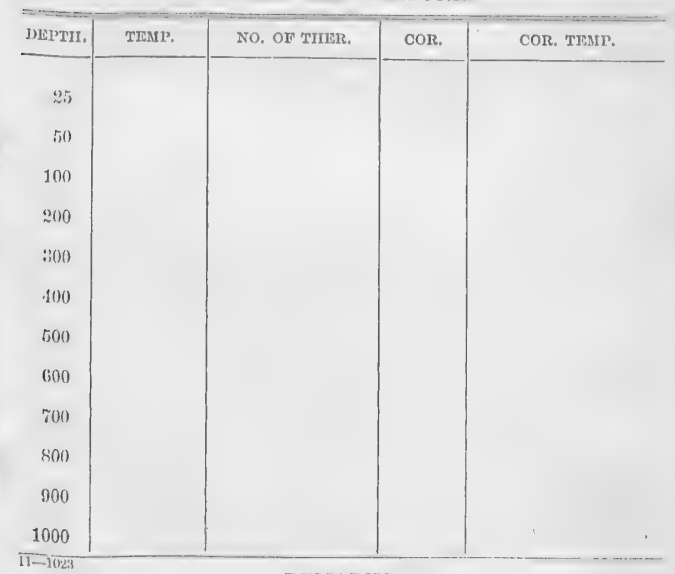

REMARKS: 
No.

C $17.2 \%$

Date

$11+\pi, \ldots=$

Reel.

Turns Cor.t Depth 242215 .

Shot or-zead $60 \%$ ma T.C.T.

Bottom dk gy Fla Op. Juanm Rad.

Bottom temperature

No. of thermometer 1080 -3 Cor.

Corrected temperature

Air $8 \%^{\circ}$ Surface 8/ Drift

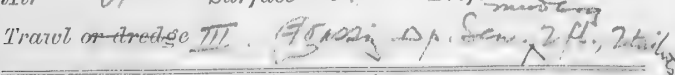

SOUNDING WIRE.

Down. atr.up.

8. cet.

5.5

$\therefore 6+1$

$0\}_{1}^{1}=1$

ㅁ‥ 1-

$\therefore, c$

$1-.20$

, .

$\because, 19$

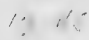

I U

10! \&

is.

'大.

17.4

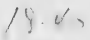

$1: 1$,

20,4

$21.1)$

27.5

7. 4.00

2505

26.15

27.2,

1630

?. $: 28.5$
54.00

$\therefore 7.15$ r.

55.25

(t), 17

10.00

$+2 ; 1)$

$+1,4 / 1$

-

44.23

L/i, ?

47.115

$1 . .135$

14x

$4: 25$

$4 / 2,10$

(10. $(1)$

$49.2^{7} 0$

98.17

$110.5 \mathrm{~s}$

35.20

a? $0=$

;2. il,

$\$ 1.10$

?. 20.6
TUIRS:

DREDGE ROPE.

0

100

200

300

400

500

600

700

800

900

1000

1100

1200

1300

1400

1500

1000

1700

1800

1000

2000

2100

2200

2300

2400

$24: ?$

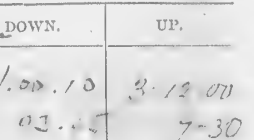

\begin{tabular}{r|r} 
am Down. & \multicolumn{1}{c}{ U1. } \\
11.01 .10 & 3.12071 \\
11... & $7-30$
\end{tabular}

$07.20 \quad 350$

10.40

$\because \cdot \ldots, y()$

16.39

20.0

27.7

26.54

70.

Us. 21

36.25

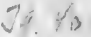

42.40

46.00

49.0

$5^{n-20}$

$5 \therefore .20$

$5 /-1 / 1$

$\therefore 7 \div 5$

$(1, \therefore 1)$

$40-50$.

औ $7-5$

$33 \div 0$

$30-50$

$27-10$

2 $\therefore \|(1)$

c) (W')

$\sqrt{-2}, \sqrt{1}$

17. 48

55.30

$\sqrt{6} .20$

$j: \because 6$

$11-20$

$12-01-3.57 \quad 7-30$

$05-30 \quad 2-33-30$

$9-15$

$12-30$

$59-50$

$56-00$

$5 /-20$

$19-051 \cdot 1-47-20$
$13 \cdot 1 / 0$

$15-45$ 


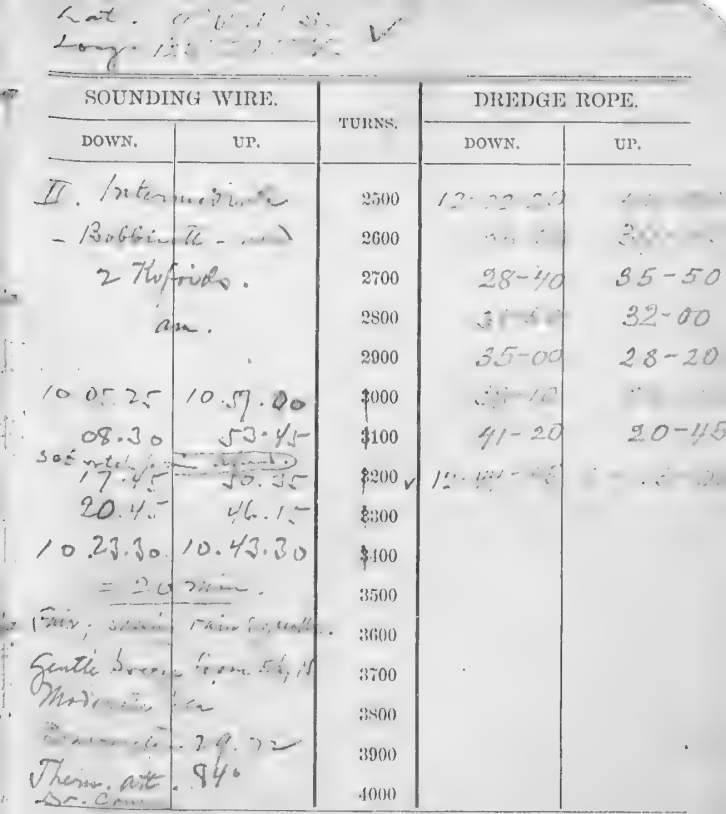

SHIRIL TEMPERA'TUREA

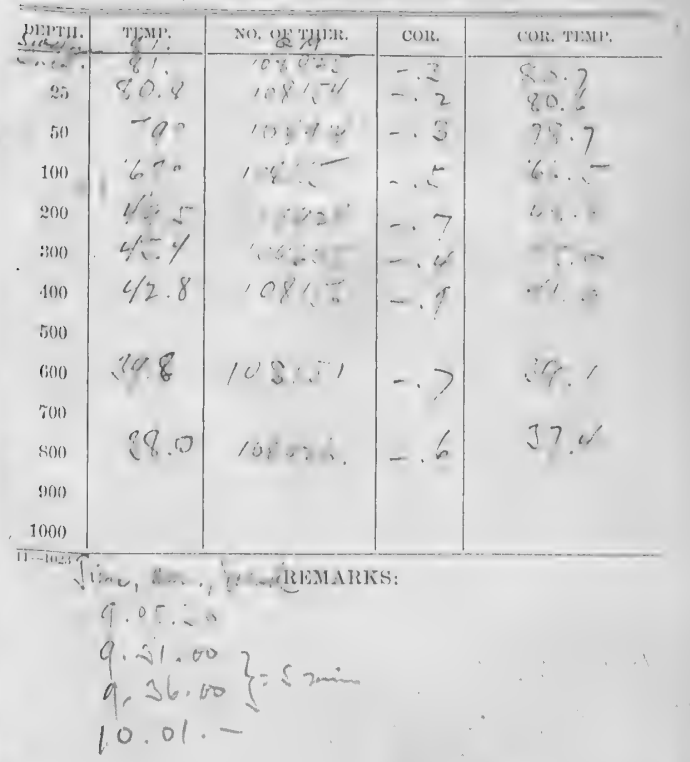


$i$.

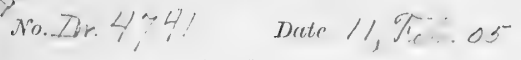
Machine. Reel.

Turns

Cor., +

Depth

Shot or lead

Bottom

Bottom temperature

No. of thermometer.

Corrected temperature

Air, $84^{\circ}$

Surface

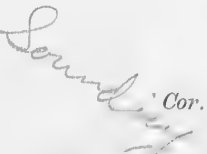

Trawt or dredge

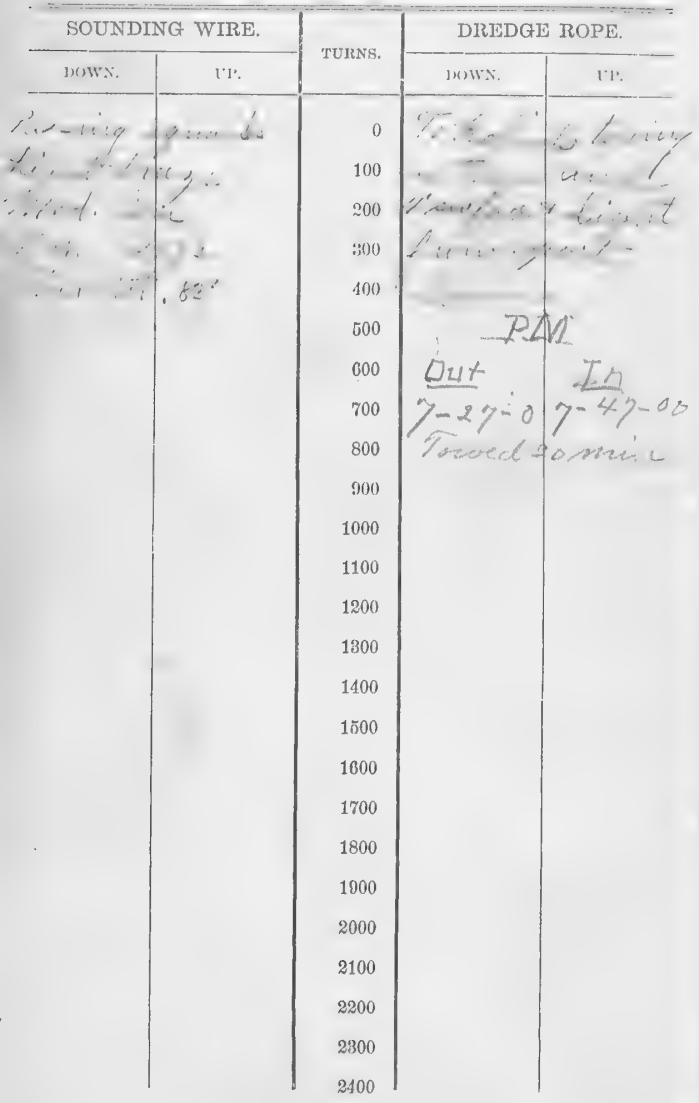




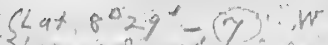

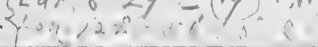

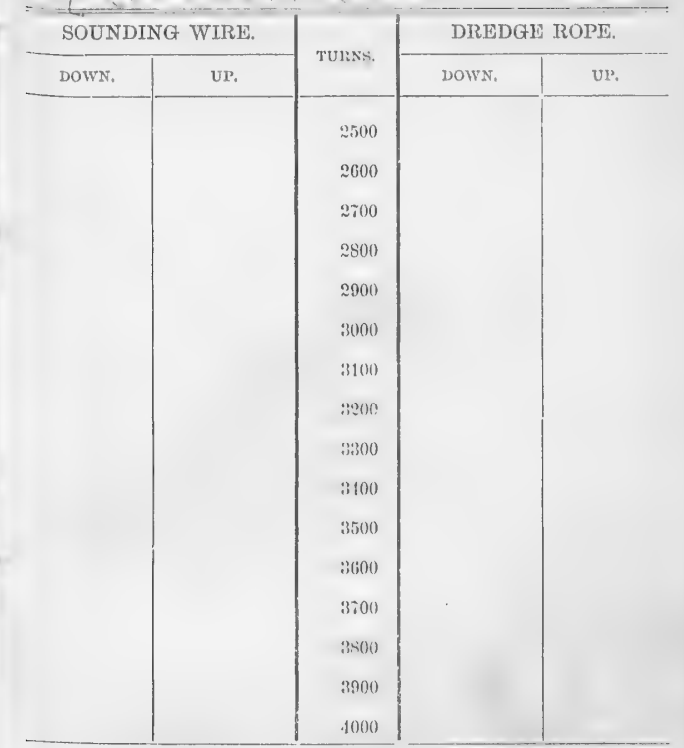

SERIAI TEMPERATURES

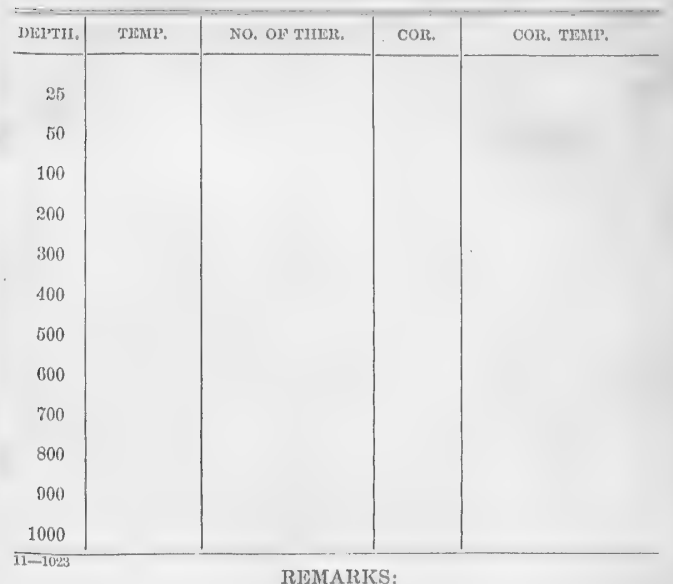

REMARKS: 


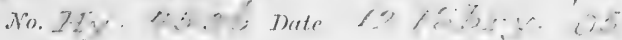
Cucas Mactine Reet.Liecas
Turns
Cor:t
Depth 2380 fuls.

shol ar-tend

Bottom No scanple browght us.

Bottom temperature $\quad 35.0$

No. of thermometer 108023 Cor.

Corrected temperature
Ait $82^{\circ}$
Surface
$80^{\circ}$
Drift

Trawl or dredse

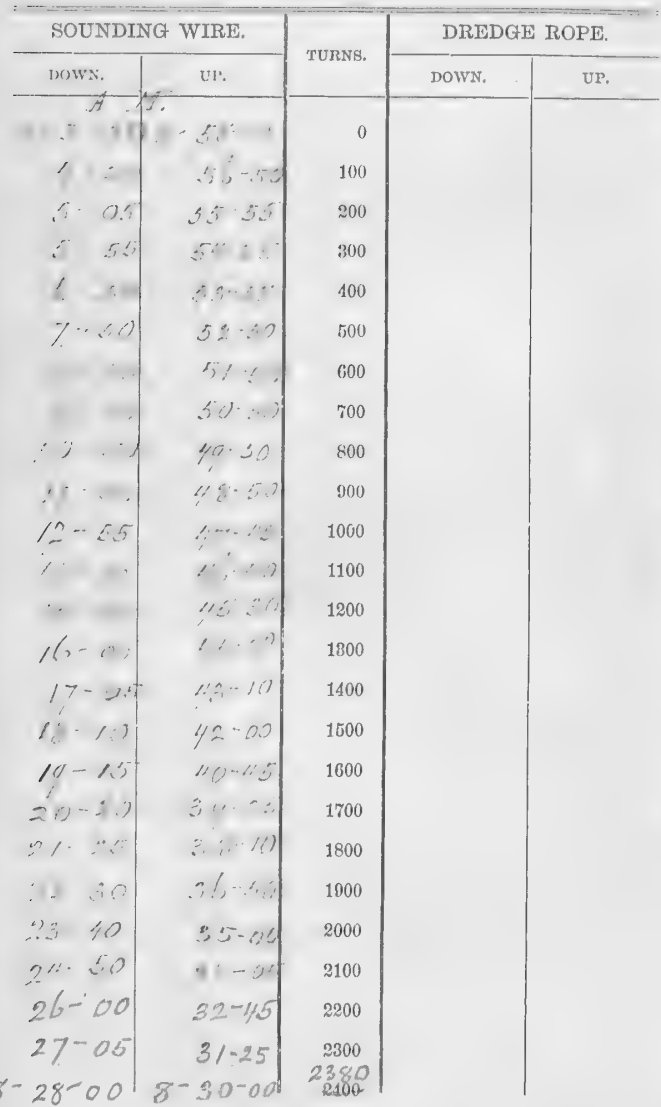


Lat. $4 . \sqrt{0} \cdot 5^{\prime} S$

Ang. $120^{\circ}$, w. ? W .

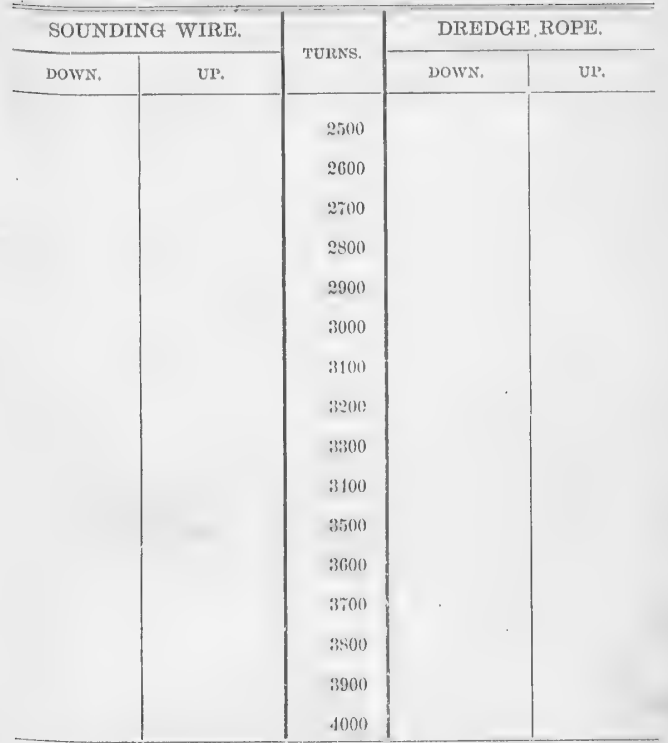

SERIAL TEMPERATURES

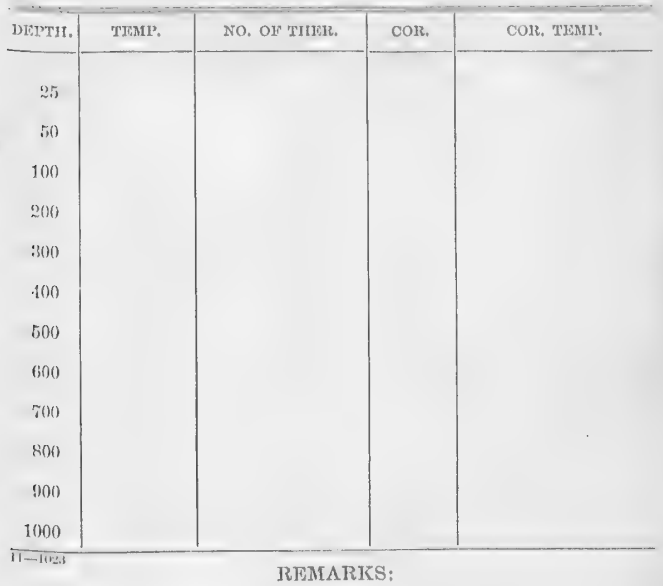




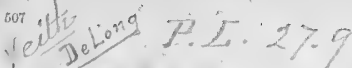

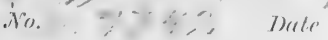

Liecai Machine. Rect. Luea.

Thurve Cortt Depth 2320 frris

shot or-lent.

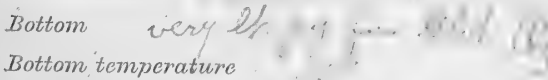

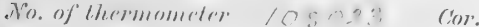

Corrected temperature

Hir D Drijl

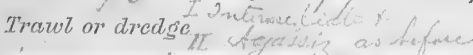

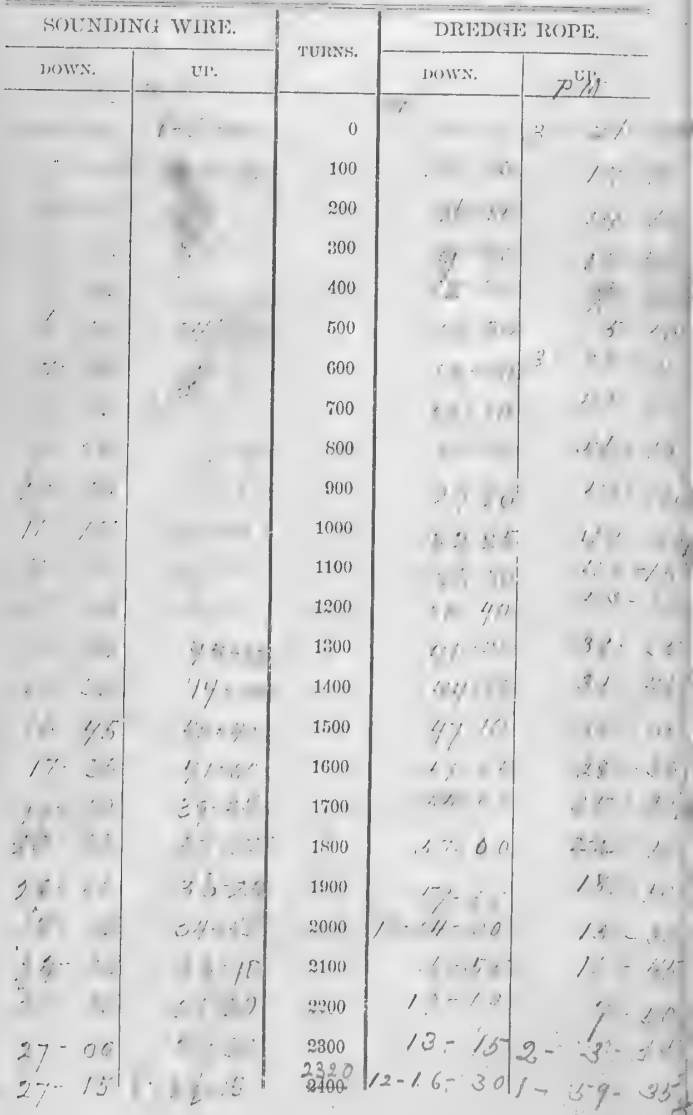


No. Dr.4\%42Coin. Date 15 t/2blos:

Machine, Reel.

Tierns

$\mathrm{Con} \cdot \mathrm{t}$

Depth

Shot or leads

\section{Bottom}

Bottom temperature

No. of thermometer .

Cor.

Corrected temperature
Ait
Surface
Drift

Thawl or dredge

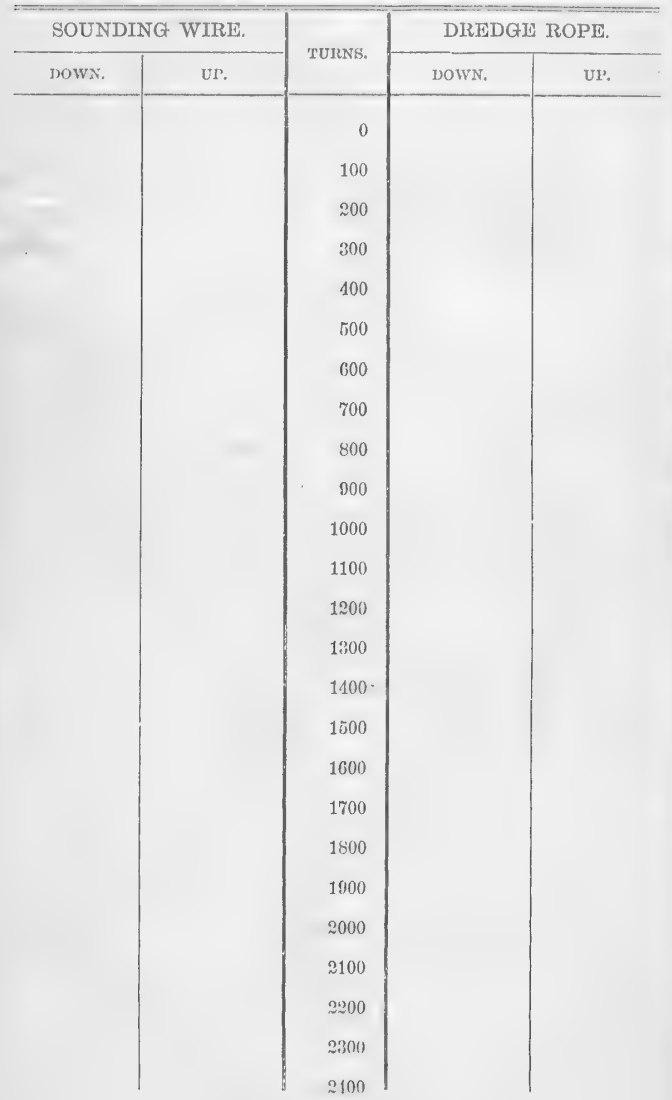




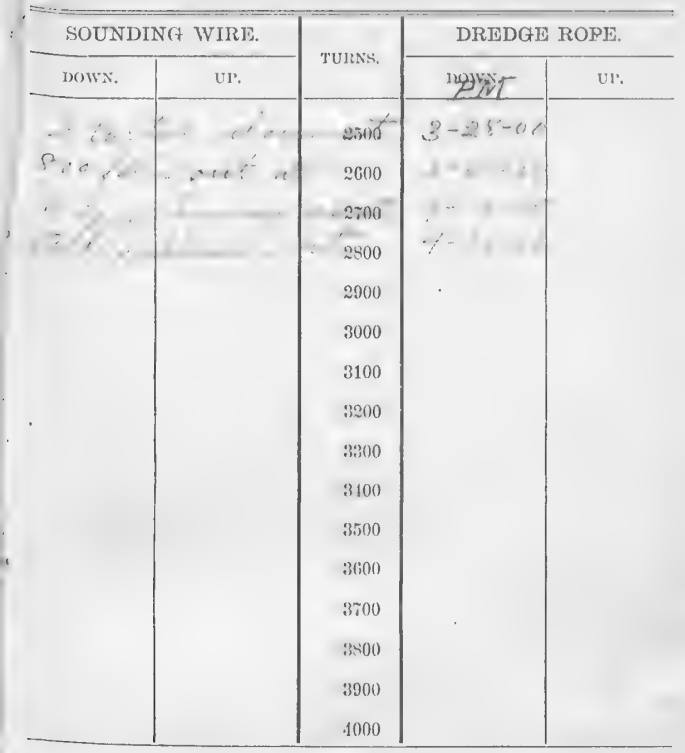

SERIAT, TEMTPRATTURES.

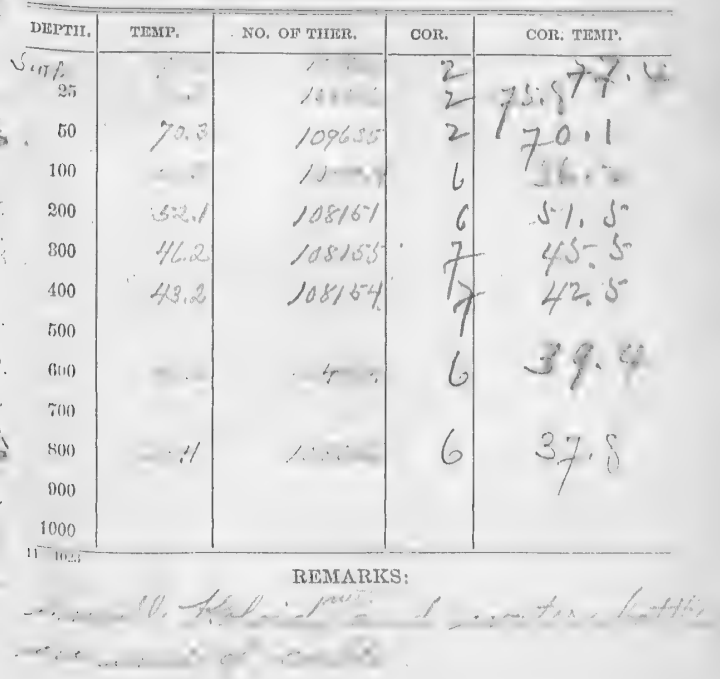


No.

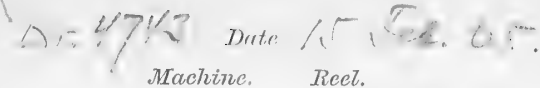

Turns Cor.t Depth

Shot or lead.

Bollom,

Bottom temperature

No. of thermometer

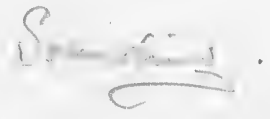

Cor.

Corrected temperature
Air 80 o
Surface
78 Drift

Trawt or dredge
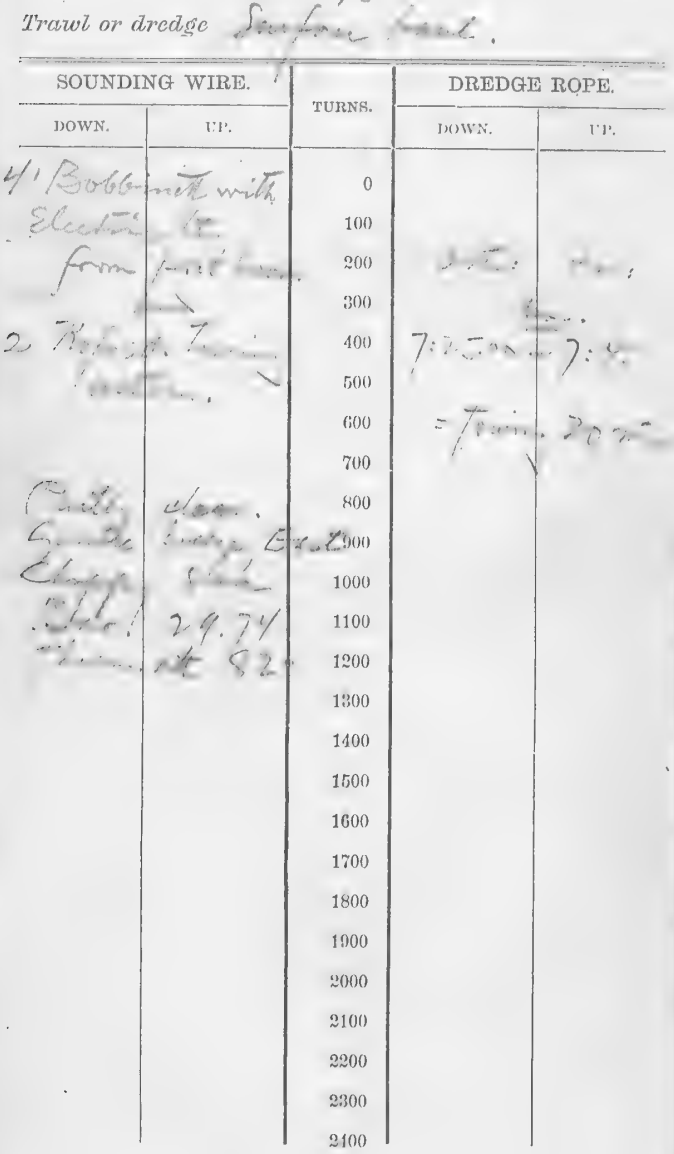


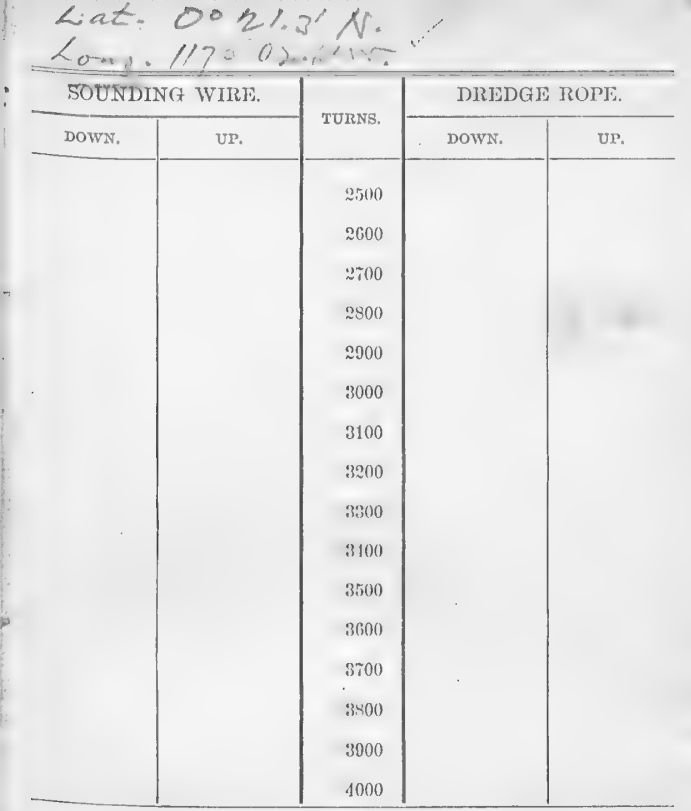

SEIRIAI TEMPERATURES.

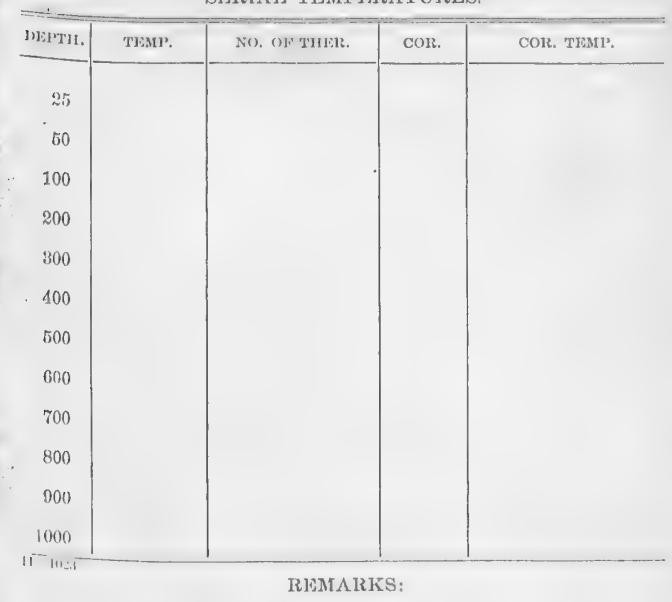


$\because$ No. H\&\#:

Derte?

Machine. Reel.

Turns Cor.t

Shot on-boud bolbe and \%.C. T rewbed

Bottom Do aluceivater

Bottom temperature $3,5,1^{\circ}$

No. of thermometer / 08023

Cor. $\quad 7$

Corrected temperature

Air ygo Surface $78.5^{\circ}$ Drift

Trawl or dredge

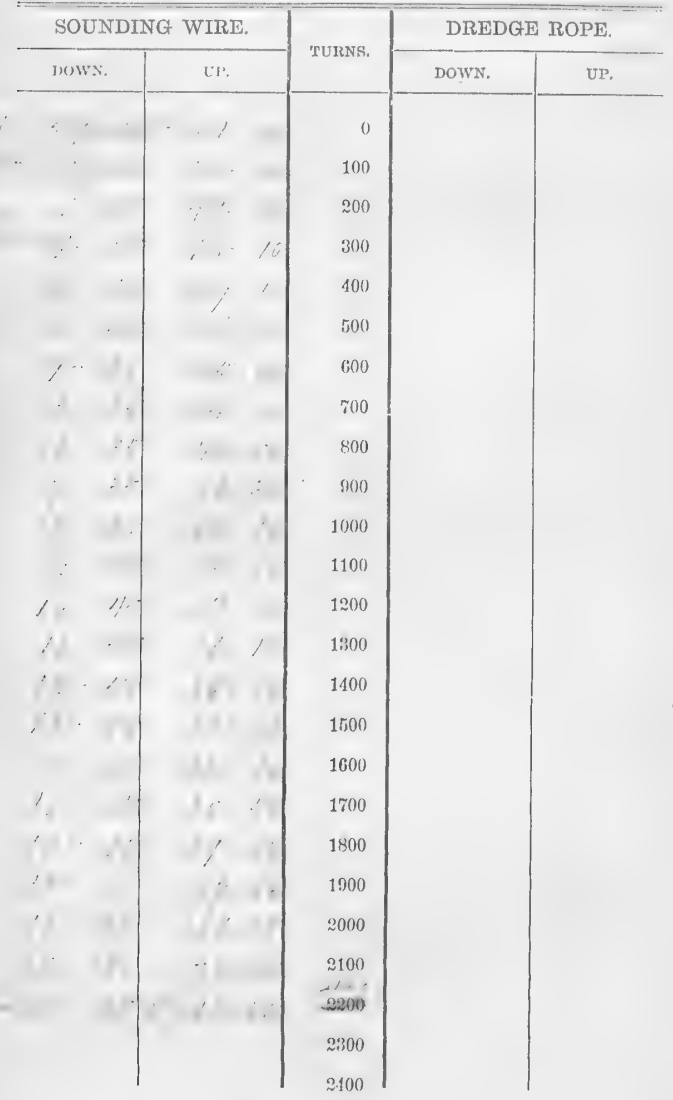




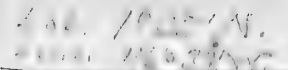

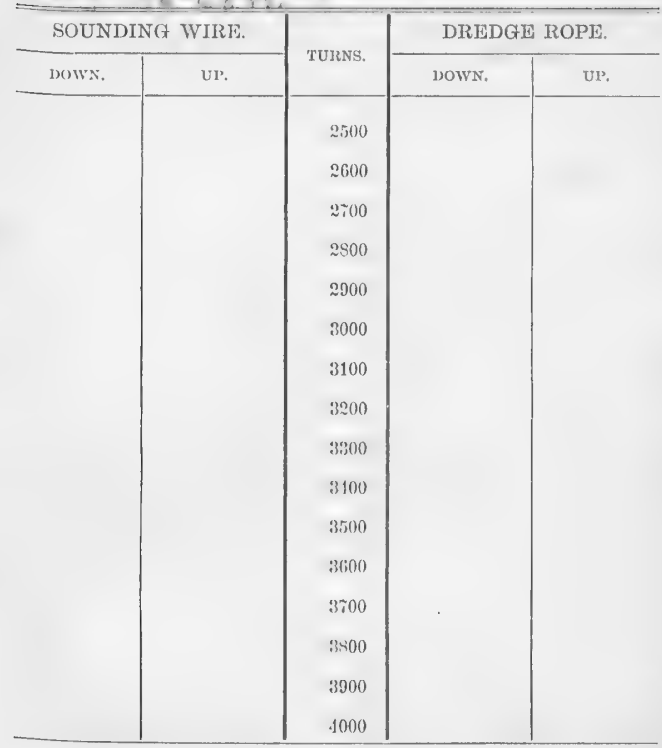

SERIAJ, TENPPEATURES.

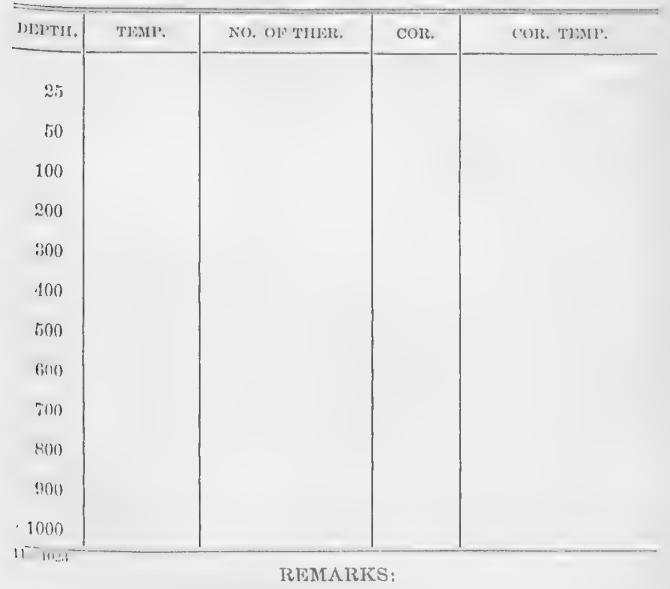


No. Ans, Jutr Olwesh Machine Reel.
Turns
Cor +
Depth $2200 \sqrt{34}$.

Shot orterert.

Bottom

dK y glo O3.

Bottom temperature

No. of thermometer 108023 . Cor.

Corrected temperature
Air
810
Surface
390
Drift

Trowl or dredge

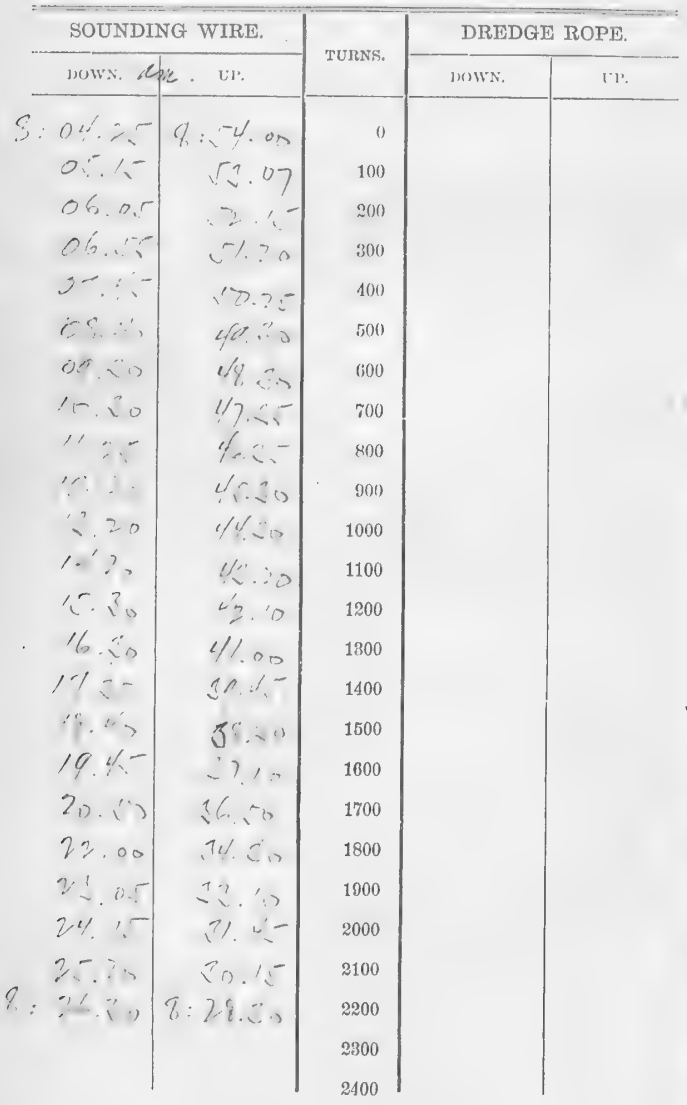




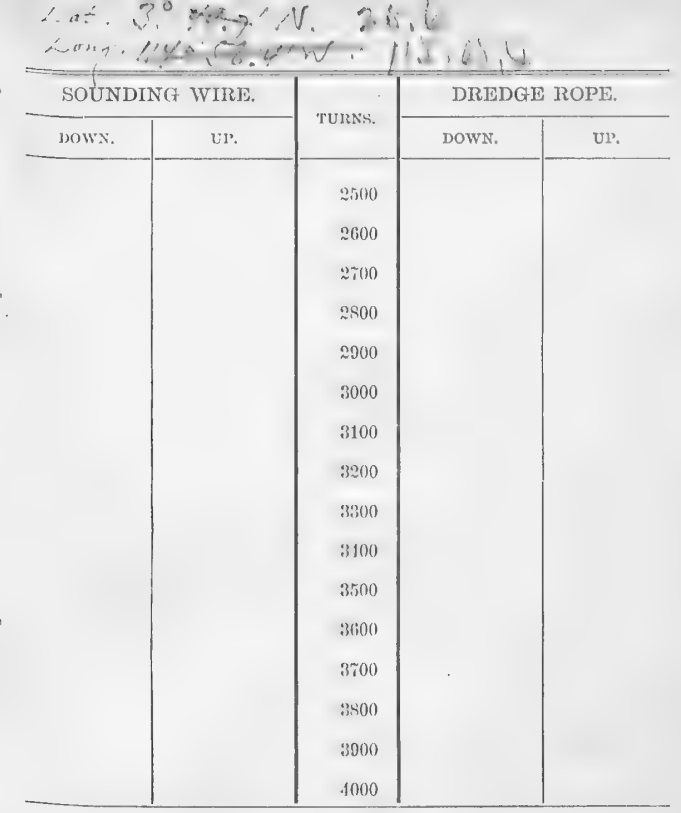

SERIAL TEMPERATURES.

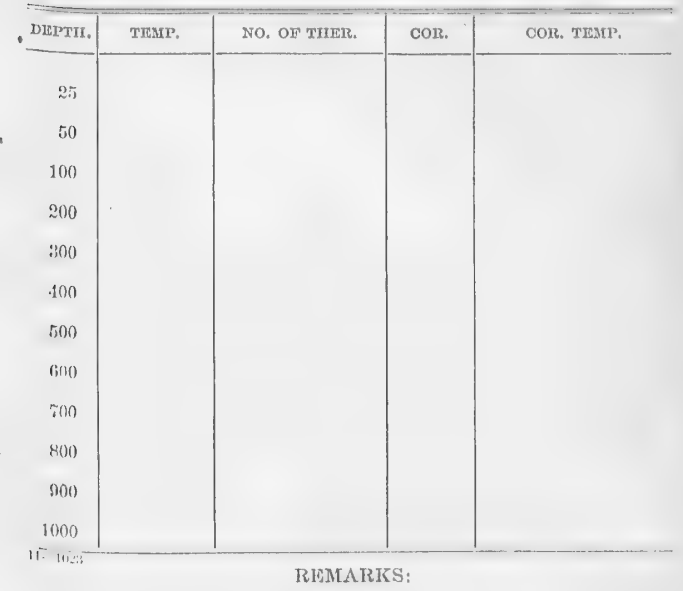


Aro. Date 18 I'ebsy '05 Sueas Machine.

Reel.

Turns Cor.t

Shot aze bead

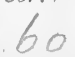

Bottom

Bottom temperature

No. of thermometer

Corrected temperature

Cor.

Air

$81^{\circ}$

Surface

80

Drift

\section{Trawl or dredse}

\begin{tabular}{|c|c|c|c|c|}
\hline \multicolumn{2}{|c|}{ SOUNDING WIRE. } & \multirow{2}{*}{ Tluss. } & \multicolumn{2}{|c|}{ DREDGE ROPE. } \\
\hline bow:. & UP. & & now. & IT. \\
\hline$\ldots$ & $8-51-00$ & 0 & & \\
\hline . & $8-38-00$ & 10 & & \\
\hline 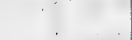 & $50-0 i$ & 100 & & \\
\hline & $47-10$ & 200 & & \\
\hline$\because 1=$ & $\cdot$ & 300 & & \\
\hline$\therefore 1 \therefore$ & $47-20$ & 400) & & \\
\hline$\cdot \cdot$ & $\because \cdots$ & 500 & & \\
\hline$\cdot>$ & $\cdots \cdots$ & 600 & & \\
\hline$\therefore$ & $\ldots \cdots$ & 700 & & \\
\hline 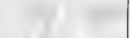 & $\therefore \therefore \quad 1$ & 800 & & \\
\hline $1 \quad 1$ & $1+1=1$ & $901)$ & & \\
\hline$\therefore \quad \therefore$ & $\therefore$ & 1090 & & \\
\hline$\therefore \quad$, & 1. & 1100 & & \\
\hline 1. & 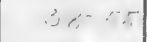 & 1200 & & \\
\hline $1 \cdots \therefore$ & $\therefore \therefore-$ & 1300 & & \\
\hline$\therefore ; \quad \therefore 1$ & $\therefore \quad \cdot$ & 1400 & & \\
\hline li & $\because \quad \cdots$ & 1500 & & \\
\hline$\therefore$, & $\therefore$ & 1600 & & \\
\hline $1 \ldots ;:$ & $\because \therefore 9$ & 1700 & & \\
\hline $1:$ & $\therefore 1 \quad \therefore \quad$ & 1800 & & \\
\hline .7. & $=0-1 i$ & 1900 & & \\
\hline$\cdots \quad \because 3$ & $\because \because \because \gamma$ & 2000 & & \\
\hline . & $\because \because \cdots n$ & $\begin{aligned} & 2100 \\
& \because \quad 17 \%\end{aligned}$ & & \\
\hline 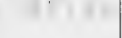 & . & 2200 & & \\
\hline & & 2300 & & \\
\hline . & & 2100 & & \\
\hline
\end{tabular}


rat. \&osJ' $N$.

Tozice $112027 \mathrm{~m}$.

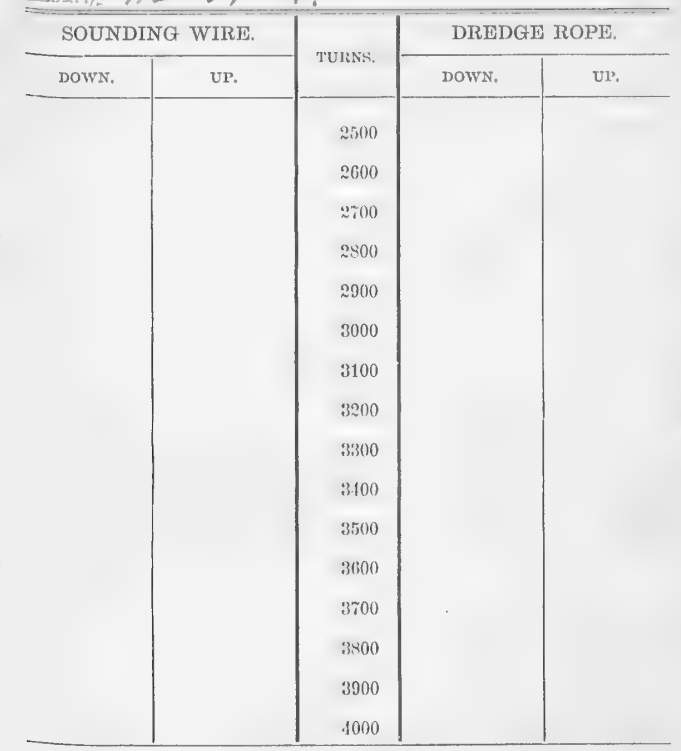

SERIAL TEMPERATURES,

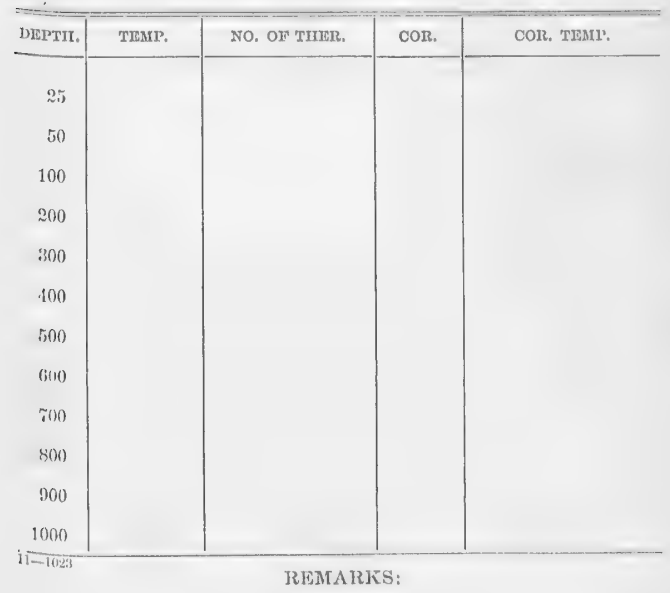


1) 4

No. $46: \ldots$

Hi: Jiate

$\bar{M}$ Tachine. Reel.

Turris Cor.t Depth 2225 fath

Shot on tewt bo liver S nafhed I

Bottom Zo S pucinuel

Bottom temperature $35: 2$

No. of thermometer $108823 \mathrm{Cor}$.

Corrected temperature

Air $72^{\circ}$ Surface $80^{\circ}$ Drift

Trawl or dredge

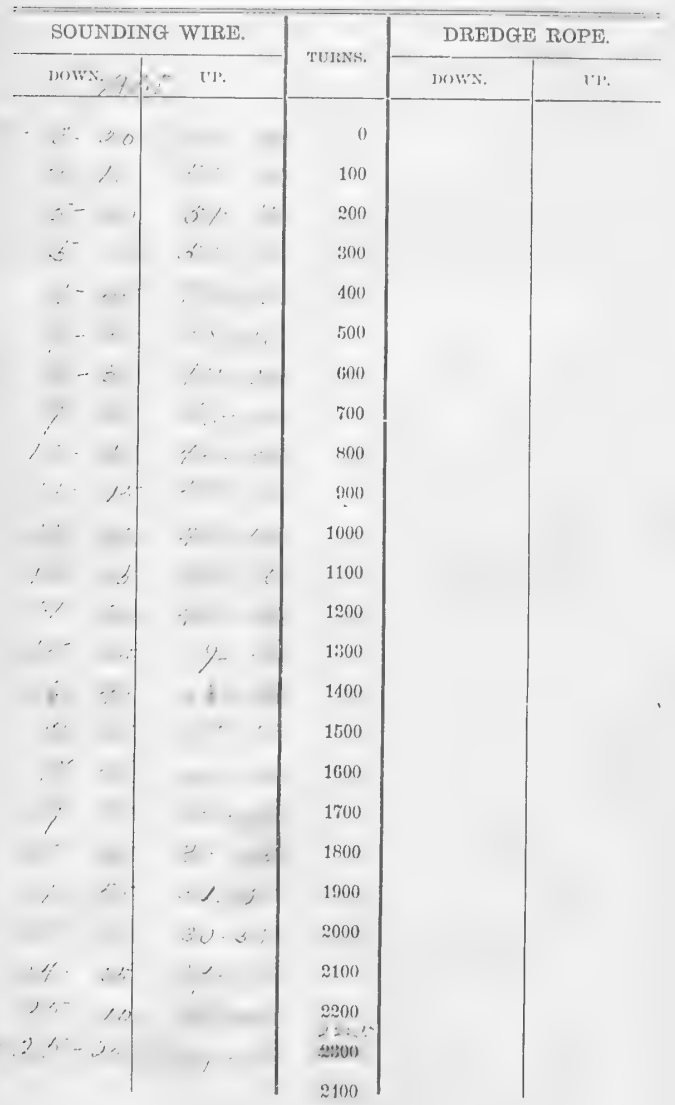




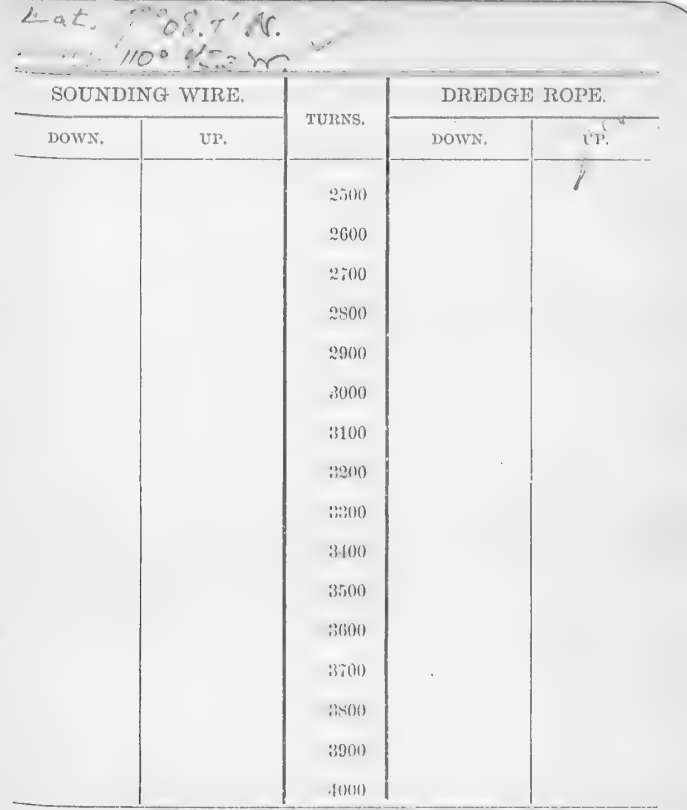

SLRLAL TUMIPRATURES

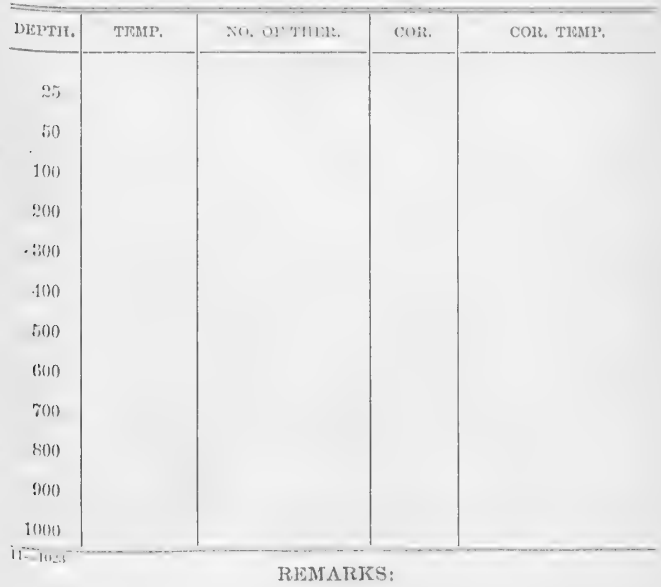


$P L=30.7 \quad 21-1023$

Whos s:4.

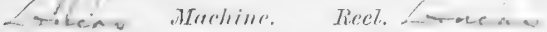
Tiur'les
Cor:. + .
Hepth $20, \pi, \hat{-2 \pi}$.

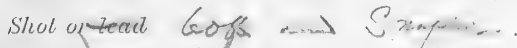

Bottom

o. $y,-d . C$.

Bottom temperature

$3 \sqrt{.} 4^{\circ}$

No. of thermometer.

108023. Cor.

Corrected temperature 347

Air 8\% - Surface $79.5^{\circ}$ Drift

Trawt or dredge

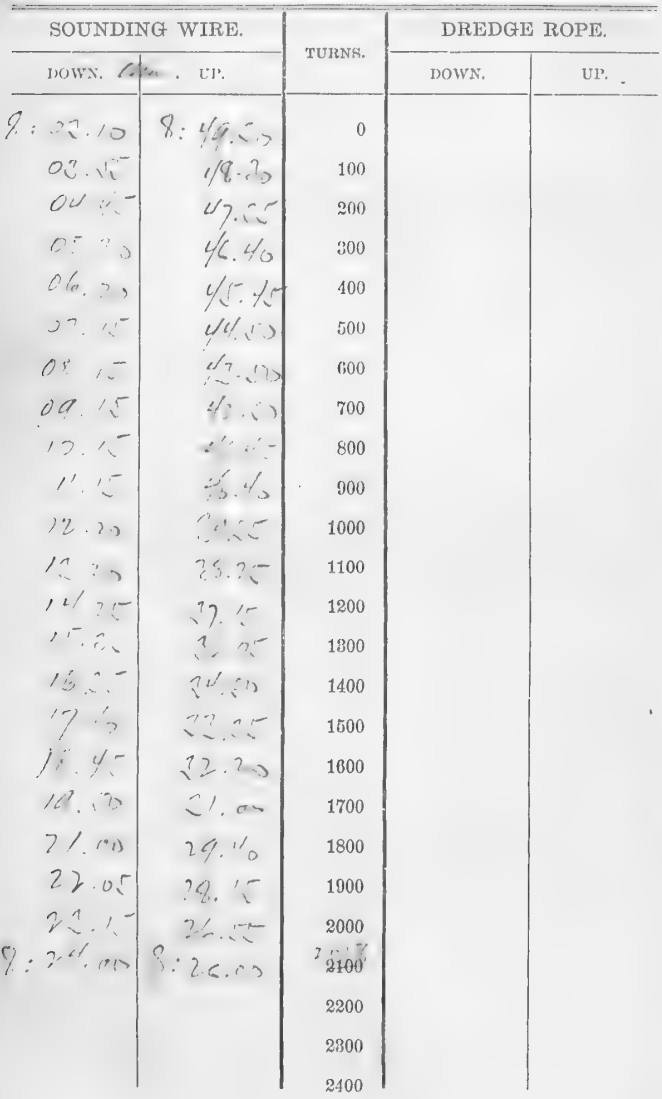


Lat. $\quad 8-52.2 \%$

$-\operatorname{erch} \cdot 108-5.4-(2)$

\begin{tabular}{|c|c|c|c|c|}
\hline \multicolumn{2}{|c|}{ SOUXNDING WIRE. } & \multirow{2}{*}{ TURNS. } & \multicolumn{2}{|c|}{ DIREDGE ROPE. } \\
\hline 1)(נw:. & l'l. & & DOWN. & UP. \\
\hline & & $2: 80$ & & \\
\hline & & 2600 & & \\
\hline & & 2700 & & \\
\hline & & 2800 & & \\
\hline & & 2900 & & \\
\hline & & 3000 & & \\
\hline & & 3100 & & \\
\hline & & 3200 & & \\
\hline & & 3300 & & \\
\hline & & 3100 & & \\
\hline & & 3500 & & \\
\hline & & $\Delta 600$ & & \\
\hline & & 3700 & & \\
\hline & & 3400 & & \\
\hline & & 3900 & & \\
\hline & & .9000 & & \\
\hline
\end{tabular}

SERIAL TEMPERATURES

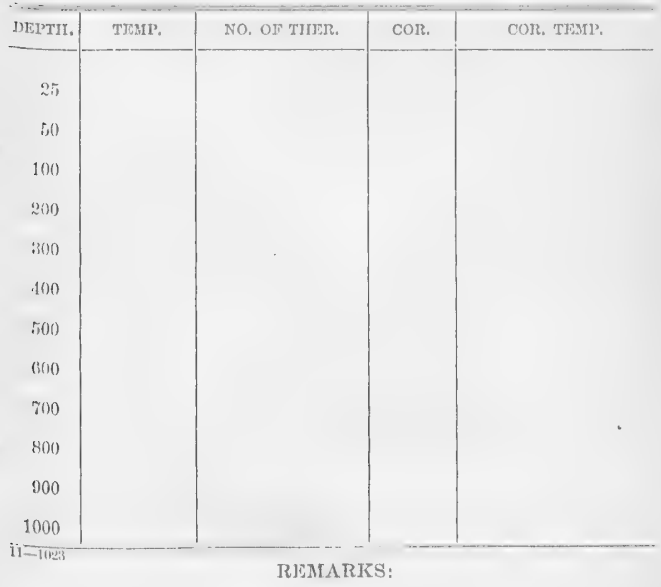


$4845 . R \cdot 2.09$

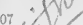

A

NQ. HE.

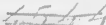

Date

21 Feb 05

Sucas

Machine.

Reel.

Turns

Cor.t

Shot ak tearit

60 th.s \& $7: 2$ ?

Depth 1955 tizs.

Bottom sitin. frm. M.

Bottom temperature $\quad 35.1^{\circ}$

No. of thermometer $108023 \mathrm{Cor}$. 7

Corrected temperature

$3 \& 4$

Air $80^{\circ} \quad$ Surface $80^{\circ}$ Drift

Trawt or dredge

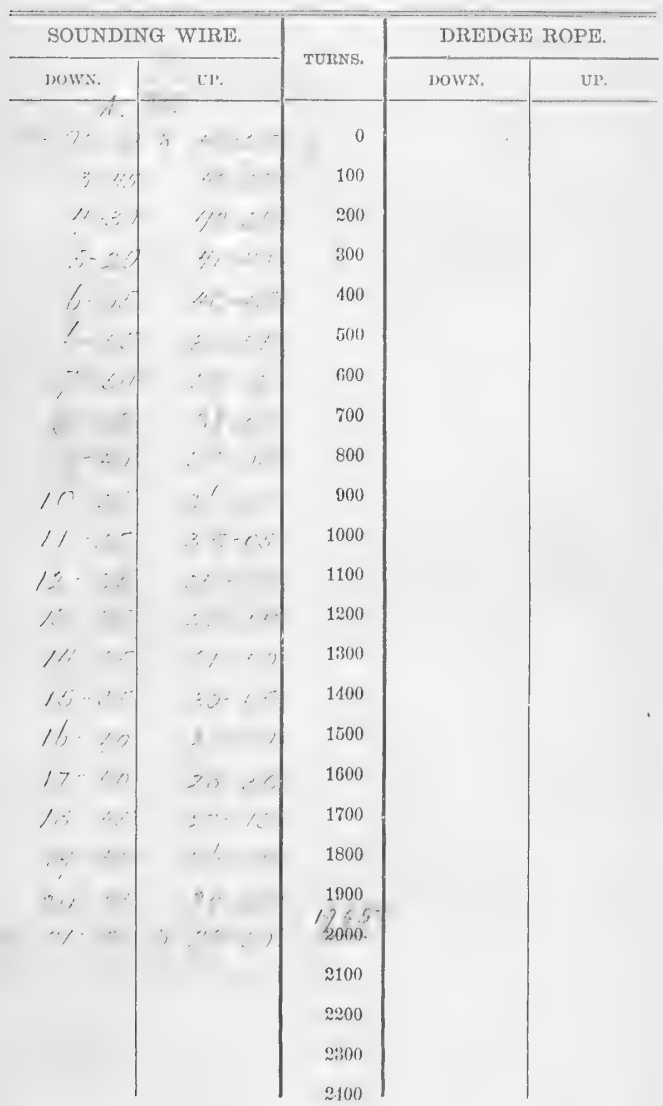




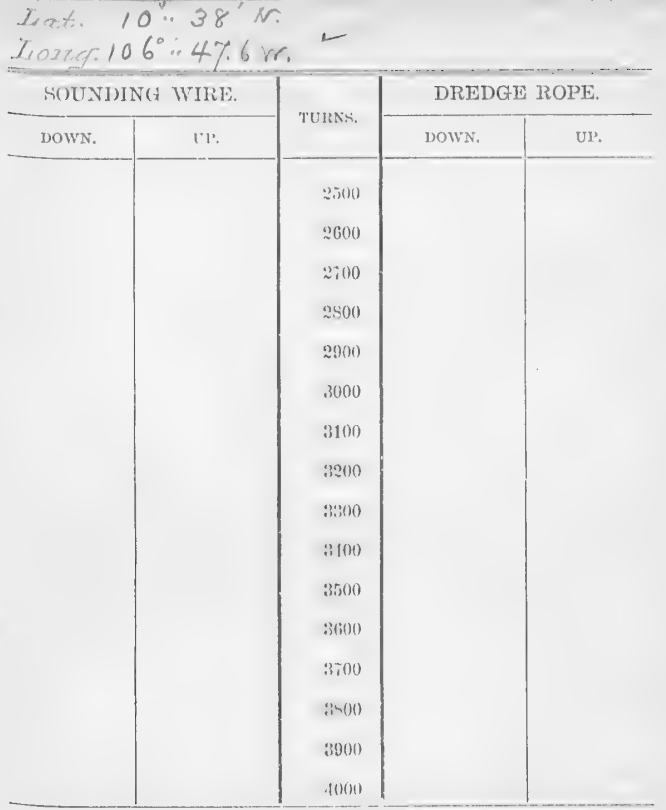

SERIAL TEMPERATURES

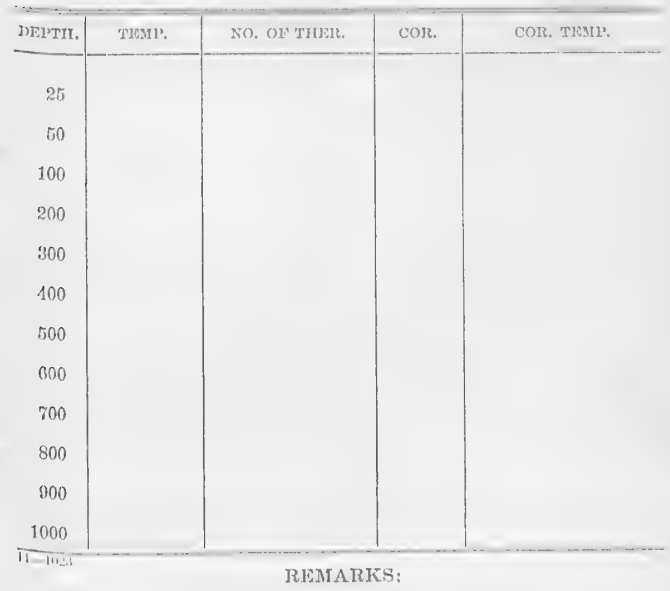




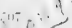

Ter

4846.

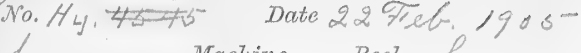
Lueree Machine Rect. Lucas
Turns
Cor, +
Depth
Shot ord teut'
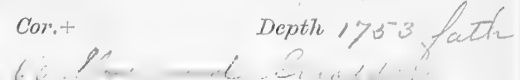

Bottom stip brie is.

Bottom temperature $33: 6^{\circ}$

No. of thermometer 108023 Cor.

Corrected temperature $34 \%$

Air $82^{\circ} \quad$ Surface y $9^{\circ}$ Drift

Trawl or dredge

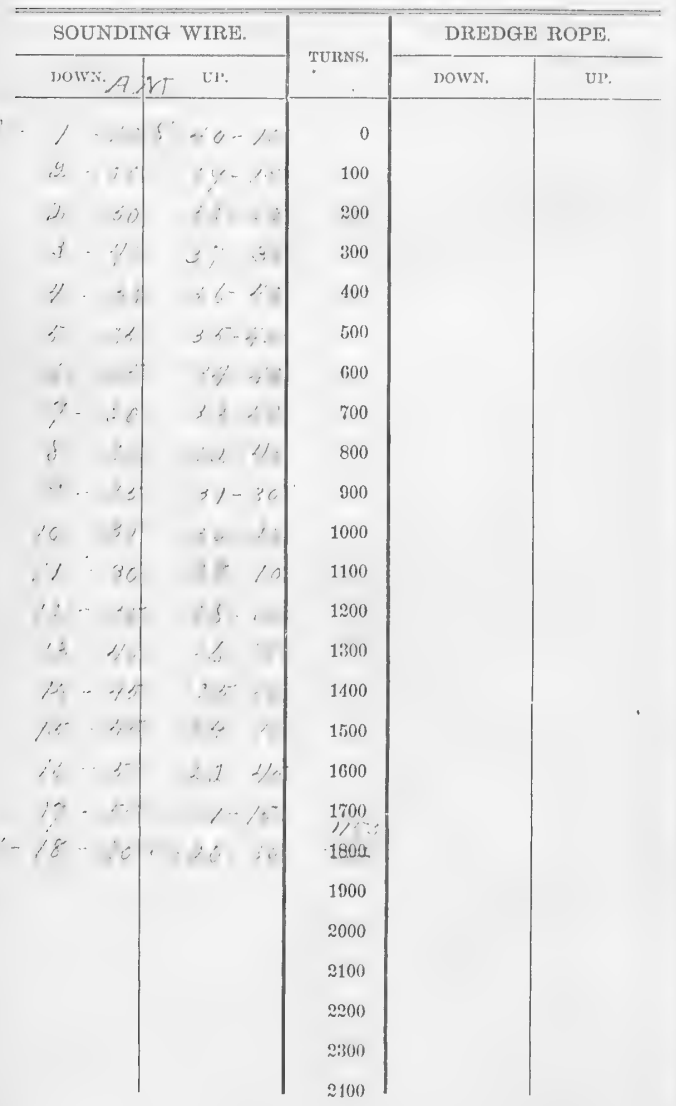


entis

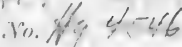

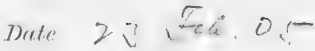

Limearo

Turns

Cor.t

Reel. Lowers

Shot on dareet la or

Bottom stic, br. 7.

Joblom tempereture

No. of thermometer

Corrected temperature

Depth 2050 ther.
Air
$80^{\circ}$
Surface
8/0 Drift

Trawl or dredge

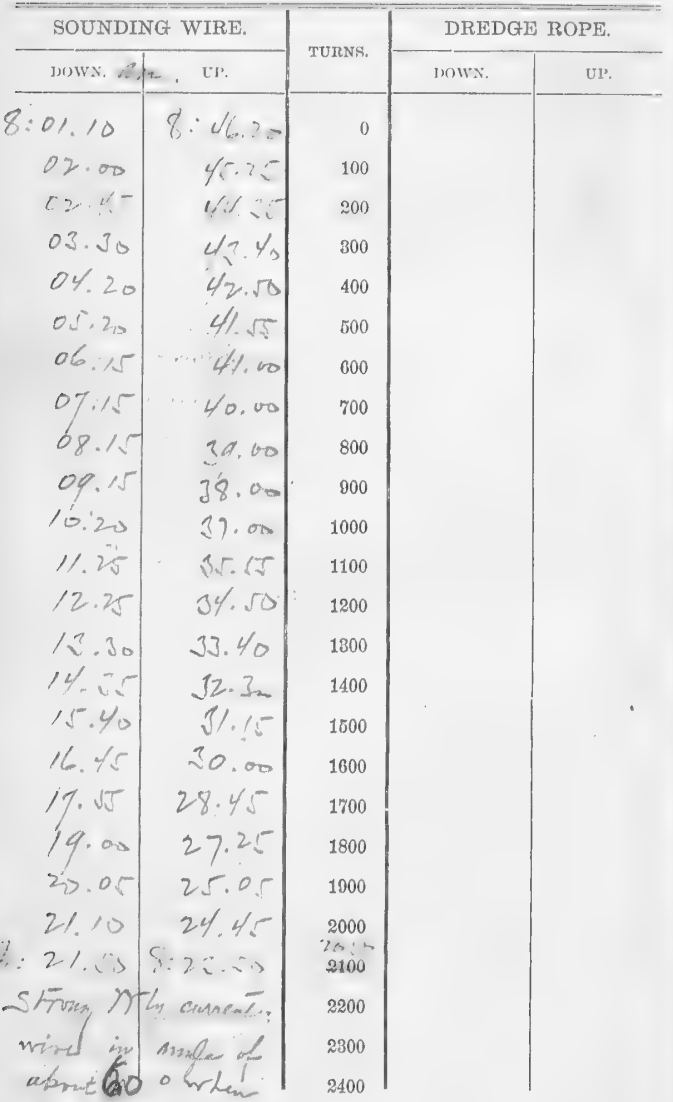

r. 2 . 
Lat. $14050 \%$

Lman. $1010 \mathrm{~J} / \mathrm{nr}$

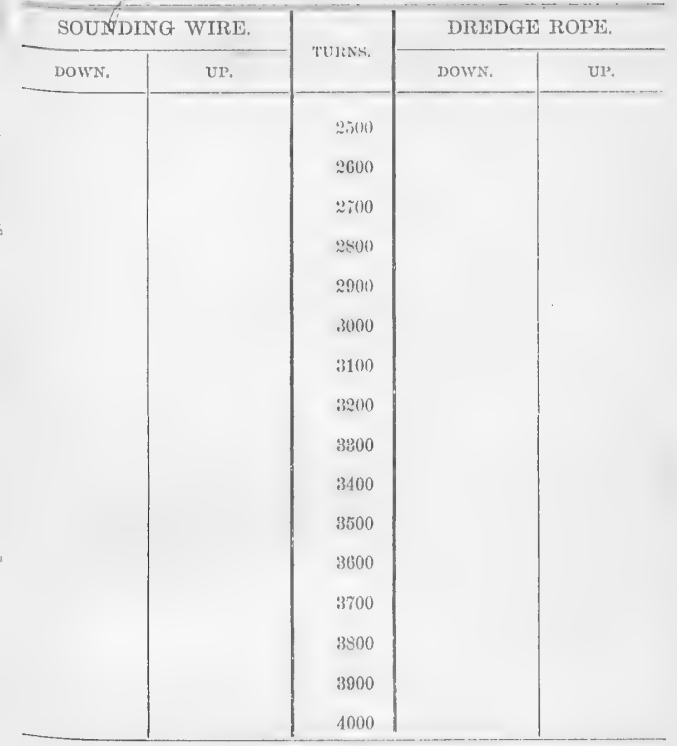

SERIAI TEMPERATURES

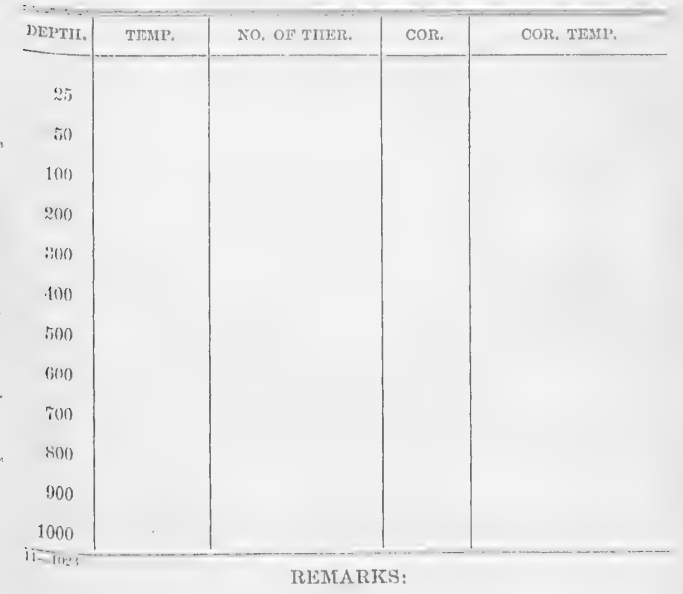


No.

Date

Machine. Reel.

Turns

Cor. +

Depth

Shot or lead

Bottom

Bottom temperature

No. of thermometer

Cor.

Corrected temperature

Aiv

Surface

Drift

Trawl or dredge

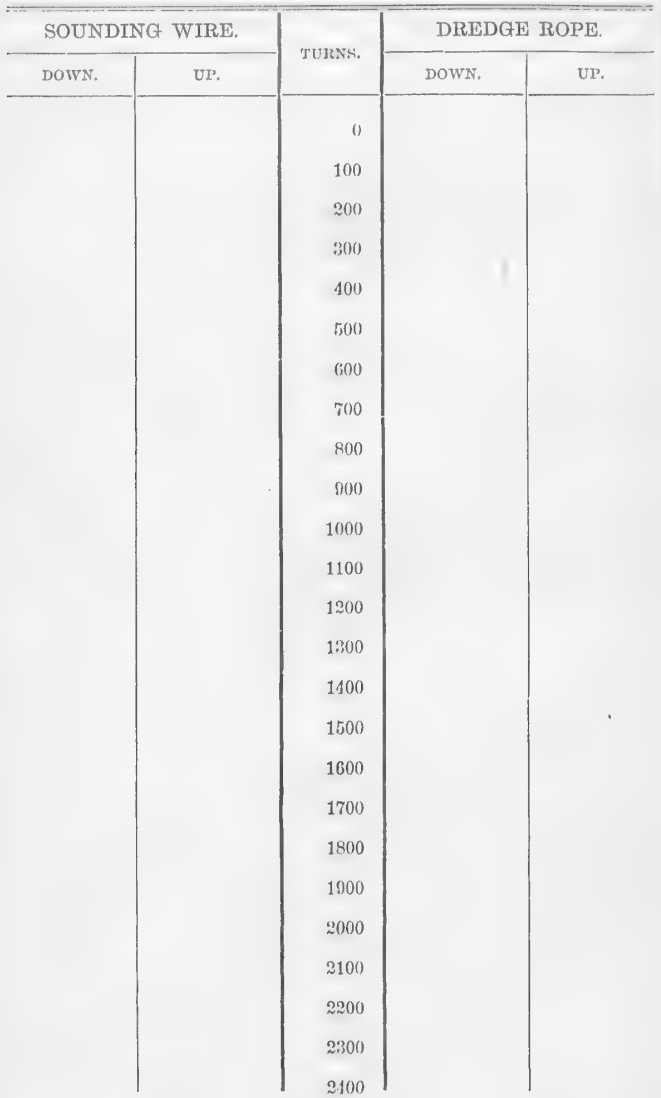




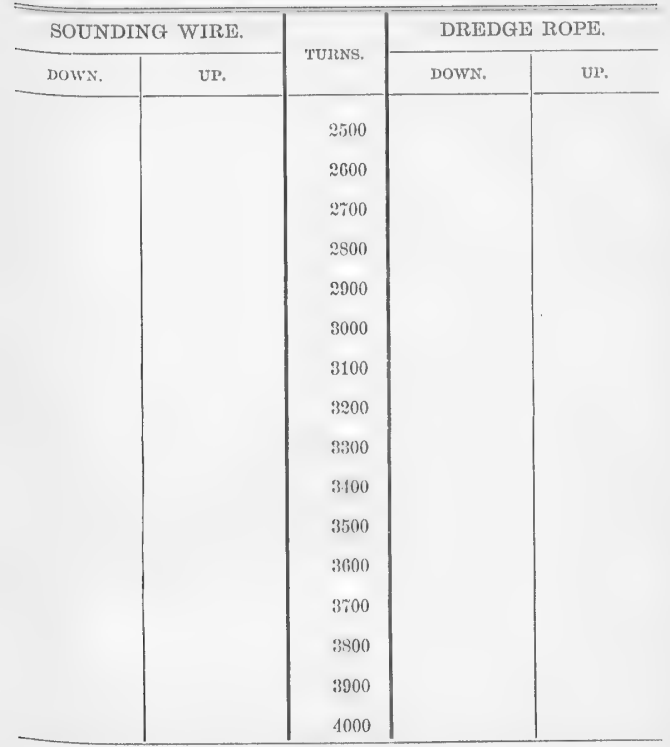

SEIRIAL TEMIPERATURES.

\begin{tabular}{|c|c|c|c|c|}
\hline I):РTII. & TLusP. & 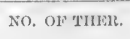 & Cor. & ('он, ткмй. \\
\hline 25 & & & & \\
\hline 50 & & & & \\
\hline 100 & & & & \\
\hline 200 & & & & \\
\hline 300 & & & & \\
\hline 400 & & & & \\
\hline 500 & & & & \\
\hline 600 & & & & \\
\hline 700 & & & & \\
\hline 800 & & & & \\
\hline 000 & & & & \\
\hline 1000 & & & & \\
\hline
\end{tabular}

REMARKS: 
$\mathcal{N o}$. Date

Machinc, Reel.

Turns

Cor? +

Depth

Shot or lead

\section{Bottom}

Bottom temperature

No. of thermometer

Cor.

Corrected temperature

Air

Surface

Drift

Thawl or dredge

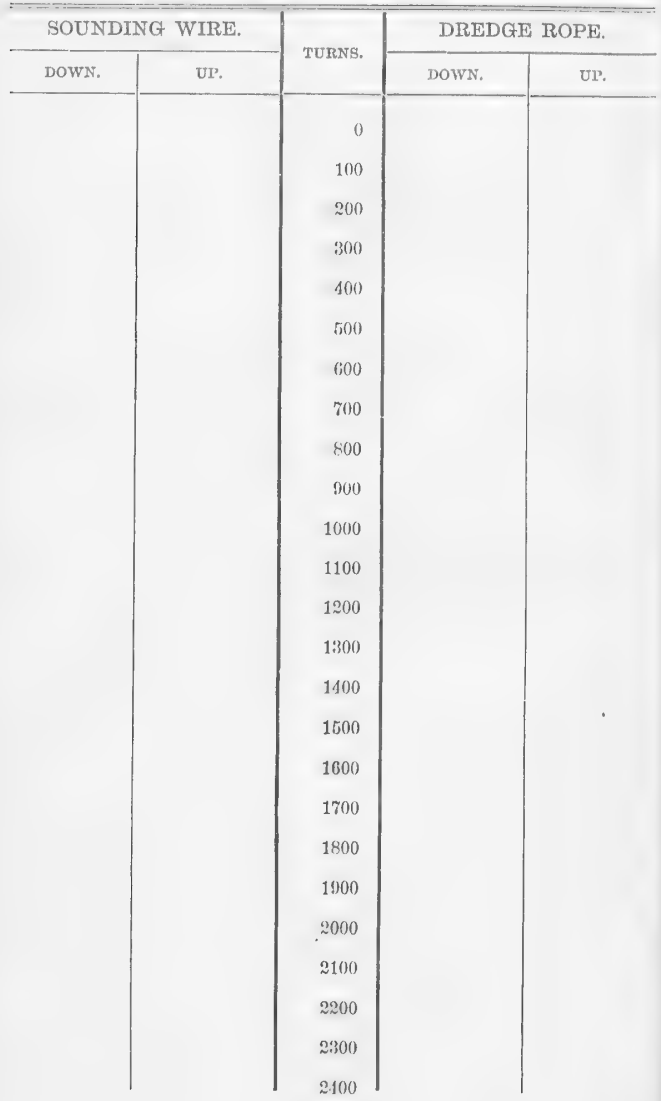




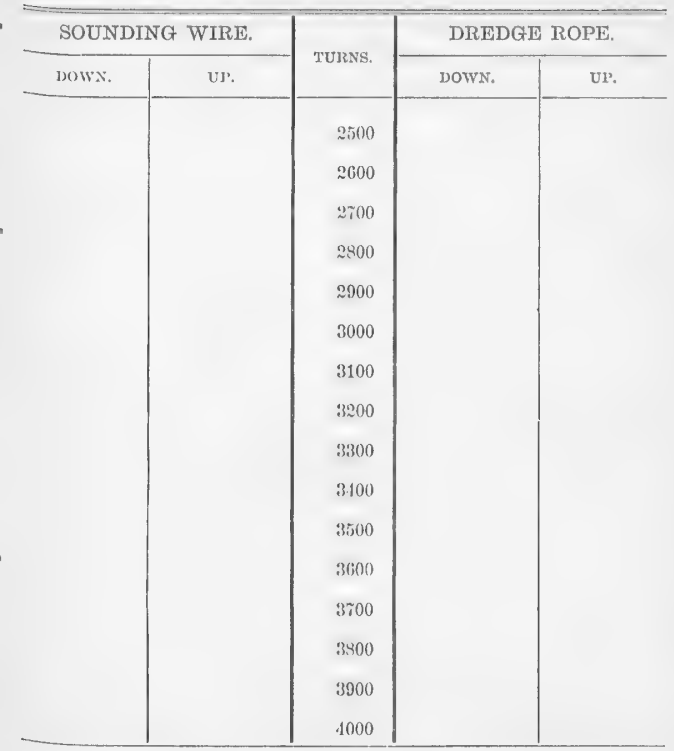

SERIAL TEMPERATURES.

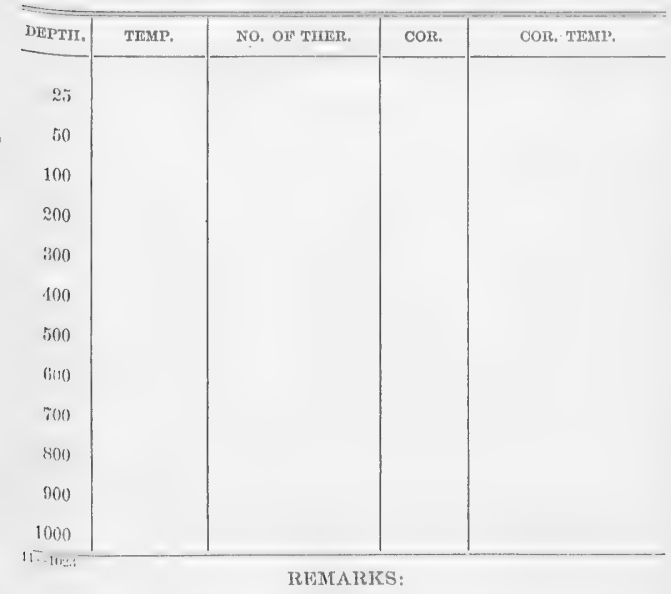


No.

Date

Machine. Reel.
Turns
Cor: +
Depth

Shot or lead,

\section{Bottom}

\section{Bottom temperature}

No. of thermometer

Cor.

Corrected temperature
Air
Surface
Drift

Thawl or dredge

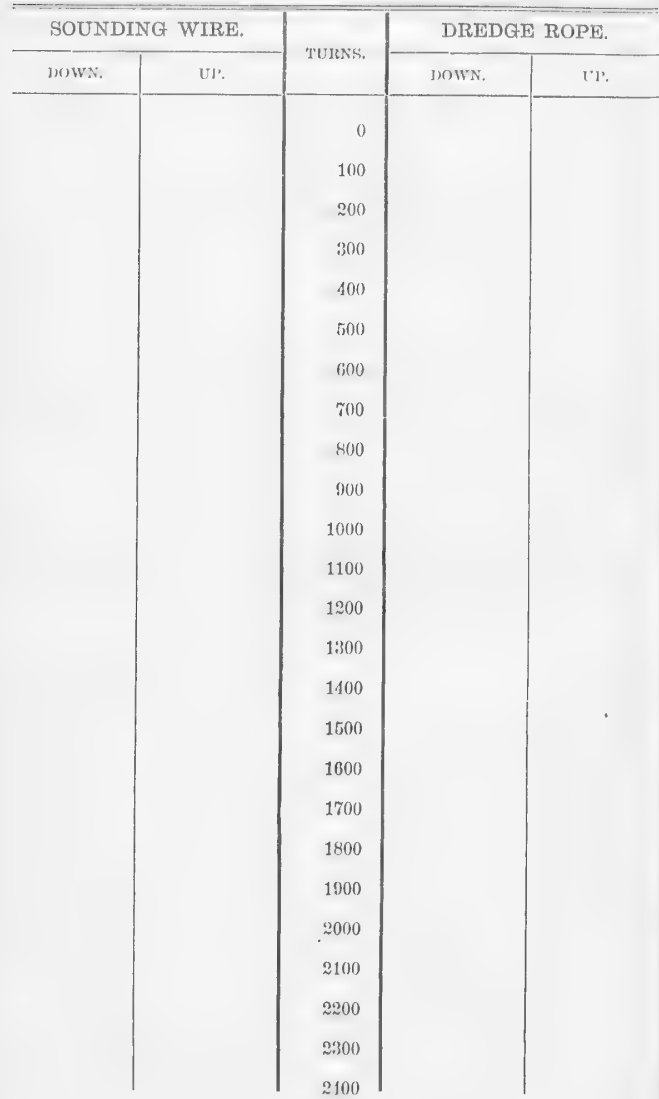




\begin{tabular}{|c|c|c|c|c|}
\hline \multicolumn{2}{|c|}{ SOUNDING WIRE. } & \multirow{2}{*}{ TUIRNS. } & \multicolumn{2}{|c|}{ DREDGE ROPE. } \\
\hline DOWN, & UP. & & DOWN. & UP. \\
\hline & & 2500 & & \\
\hline & & 2000 & & \\
\hline & & $2 \% 00$ & & \\
\hline & & 2800 & & \\
\hline & & 2900 & & \\
\hline & & 3000 & & \\
\hline & & 3100 & & \\
\hline & & 3200 & & \\
\hline & & 3300 & & \\
\hline & & 3400 & & \\
\hline & & 3500 & & \\
\hline & & 3600 & & \\
\hline & & $3 \pi 00$ & & \\
\hline & & 3800 & & \\
\hline & & 3900 & & \\
\hline & & 4000 & & \\
\hline
\end{tabular}

SERIAL TEMPERATUIRES,

\begin{tabular}{|c|c|c|c|c|}
\hline DEPTII. & TEMP. & No. (OF тUU:R. & cor. & ('OR, 'THMI'. \\
\hline $2 \pi$ & & & & \\
\hline 50 & & & & \\
\hline 100 & & & & \\
\hline 200 & & & & \\
\hline 300 & & & & \\
\hline 100 & & & & \\
\hline 500 & & & & \\
\hline ficio & & & & \\
\hline 700 & & & & \\
\hline 800 & & & & \\
\hline 900 & & & & \\
\hline 1000 & & & & \\
\hline
\end{tabular}


No.

\section{Date}

Machine. Reel.
Turns
Cor.t
Depth

Shot or lead

\section{Bottom}

\section{Bottom temperature}

No of thermometer

Cor.

Corrected temperature
Air
Surface
Drift

Trawl or dredge

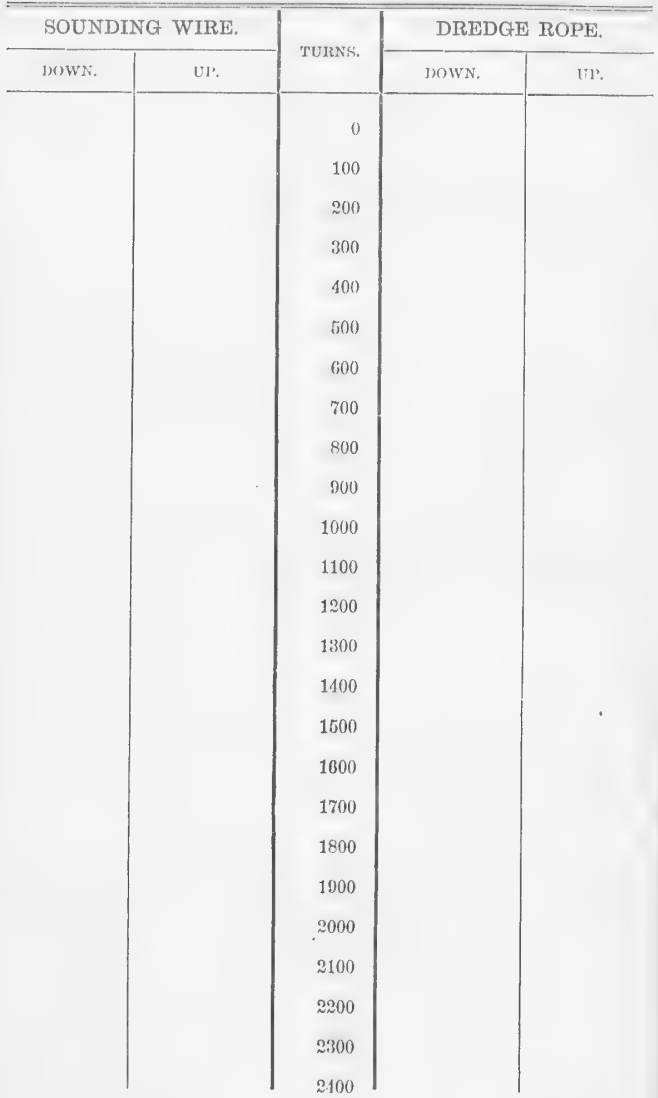




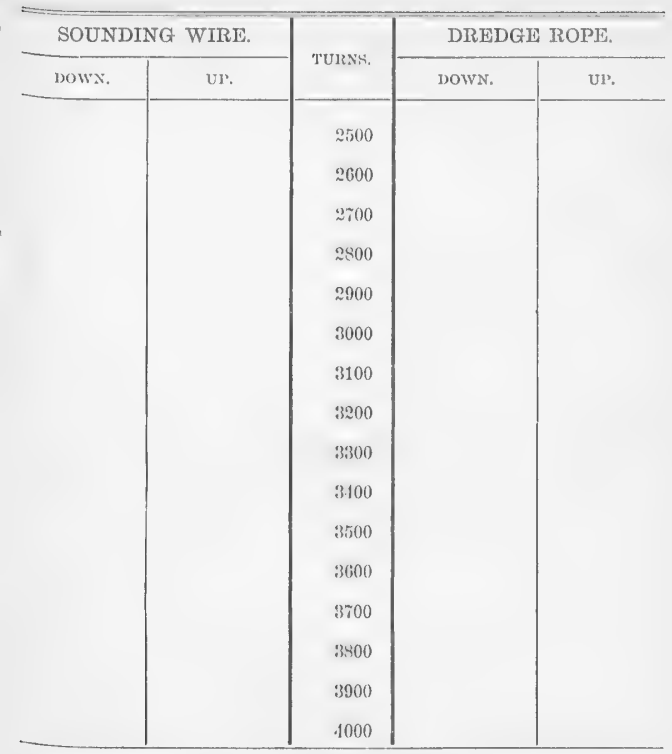

SERIAJ TEMPERATURES.

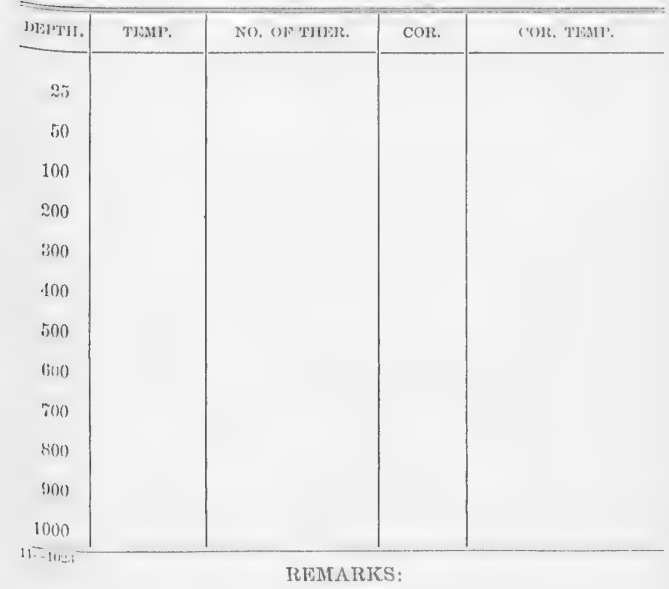


No.

Dote

Mactine. Reel.
Turns
Cor.t
Depth

Shot or lead

\section{Bottom}

\section{Bottom temperature}

No. of themometer

Cor.

Corrected temperature

Air

Surface

Drift

Trawl or dredge

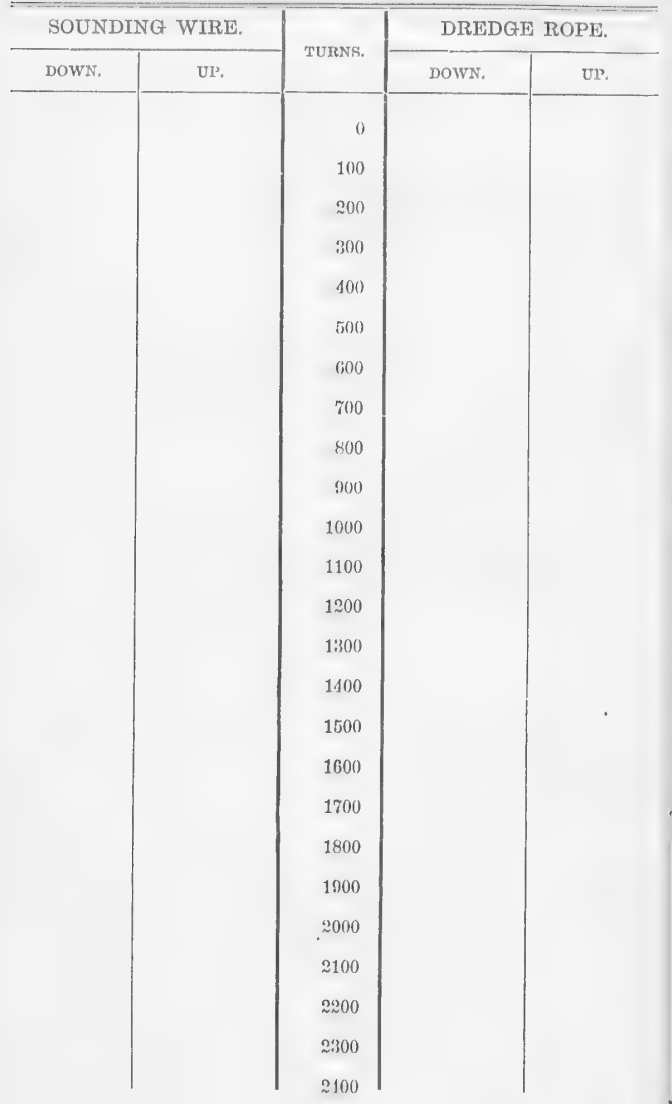




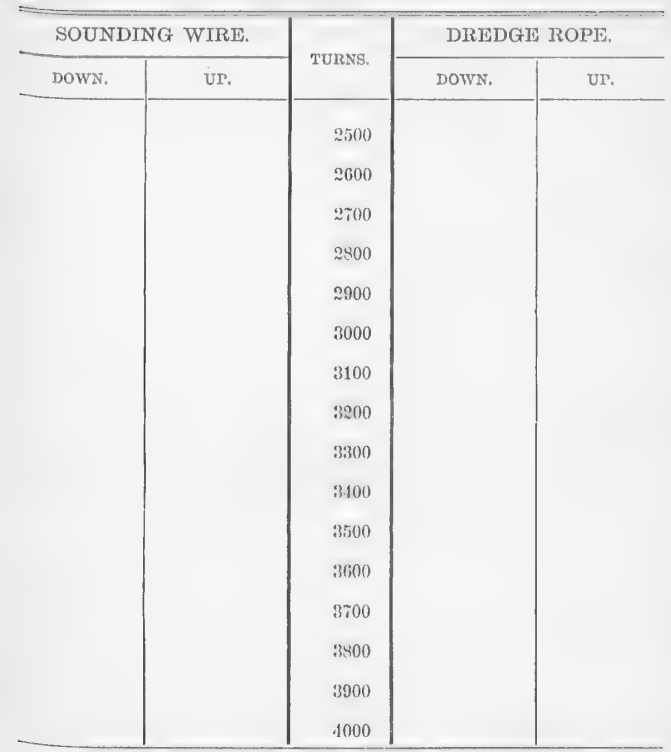

SERIAL TEMPERATURES,

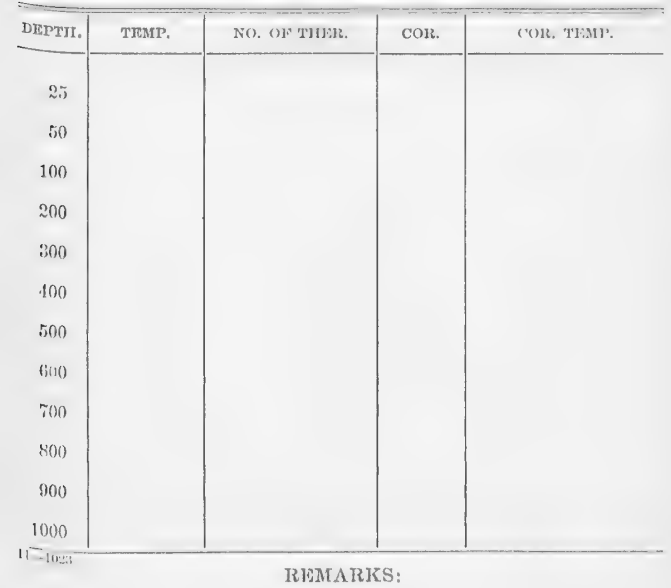


No.

\section{Date}

Machine. Reel.

Turns

Cor?.

Depth

Shot or Zeart

\section{Bottom}

\section{Bottom temperature}

No. of thermometer

Cor.

Corrected temperature

Air

Surface

Drift

Trawl or dredge

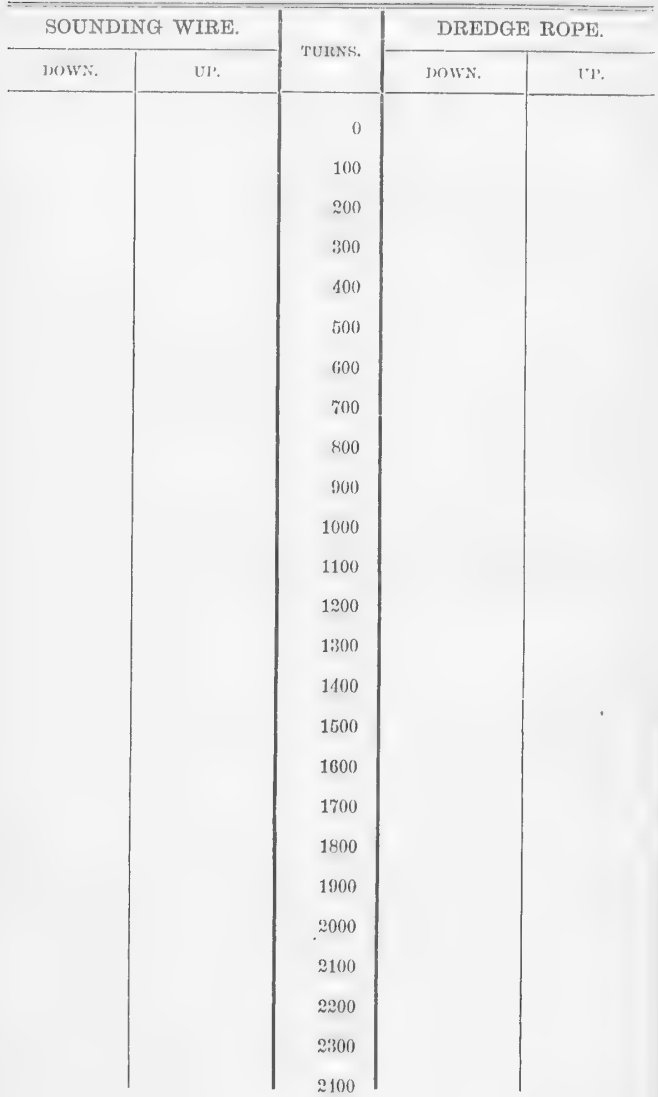




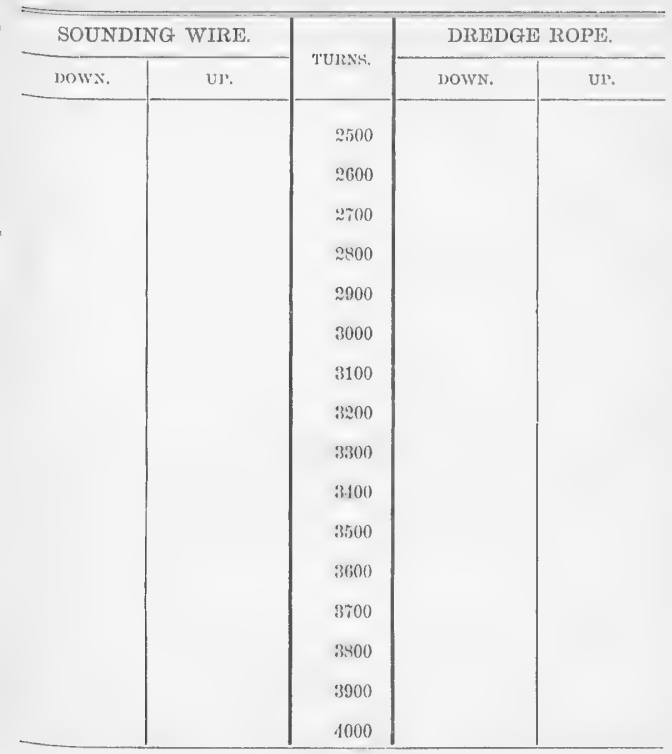

SERIAI, TEMPERATURES.

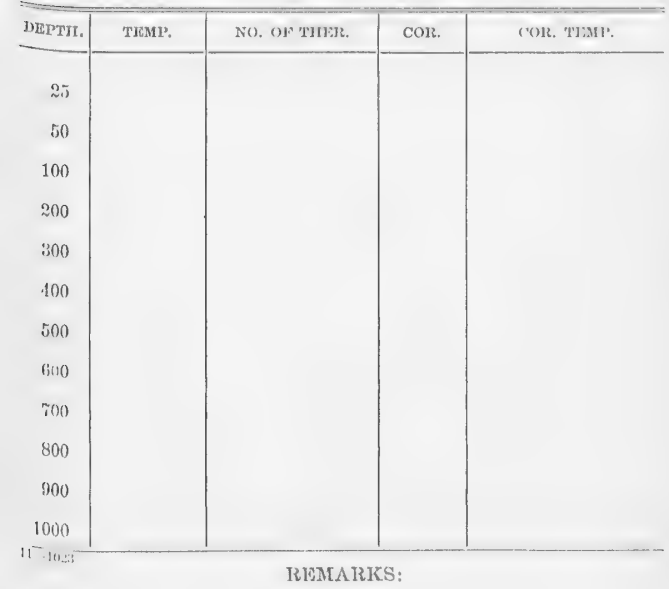


No.

Date

Machine. Recl.
Turns
Cor: +
Depth

Shot or lead

\section{Bottom}

\section{Bottom temperature}

No. of thermometer

Cor.

Corrected temperature
Air
Surface
Drift

Irawl or dredge

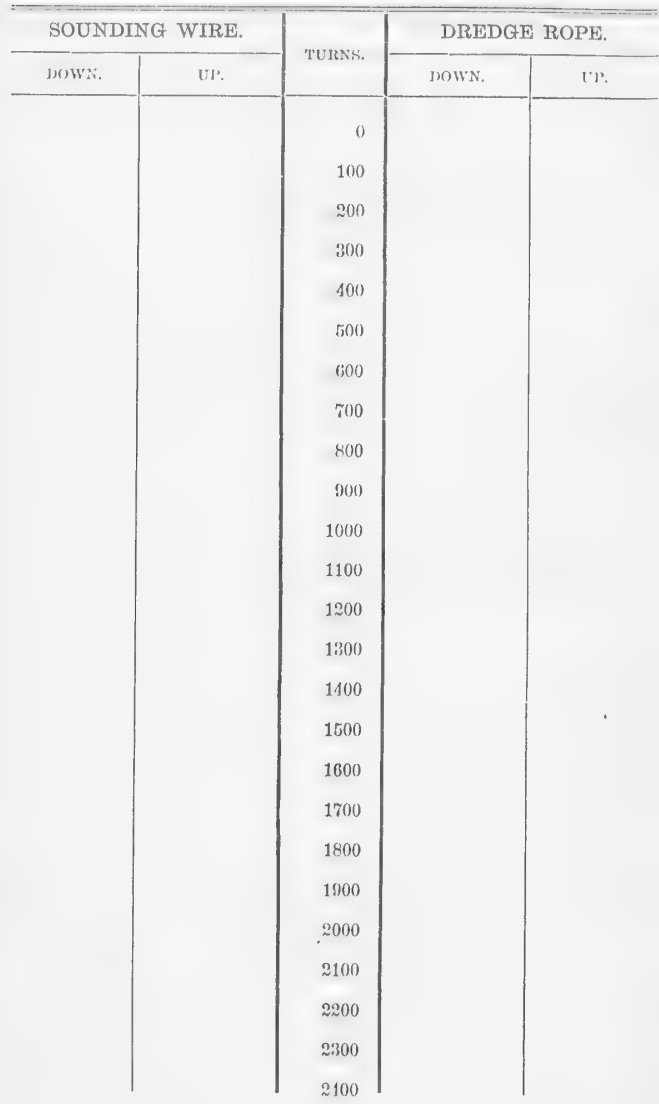


No.

Date

Machine. Reel.
Turns
Cor. +
Depth

Shot or lead

\section{Bottom}

\section{Bottom temperature}

No. of thermometer

Cor.

Corrected temperature

Air

Surface

Drift

Irawl or dredge

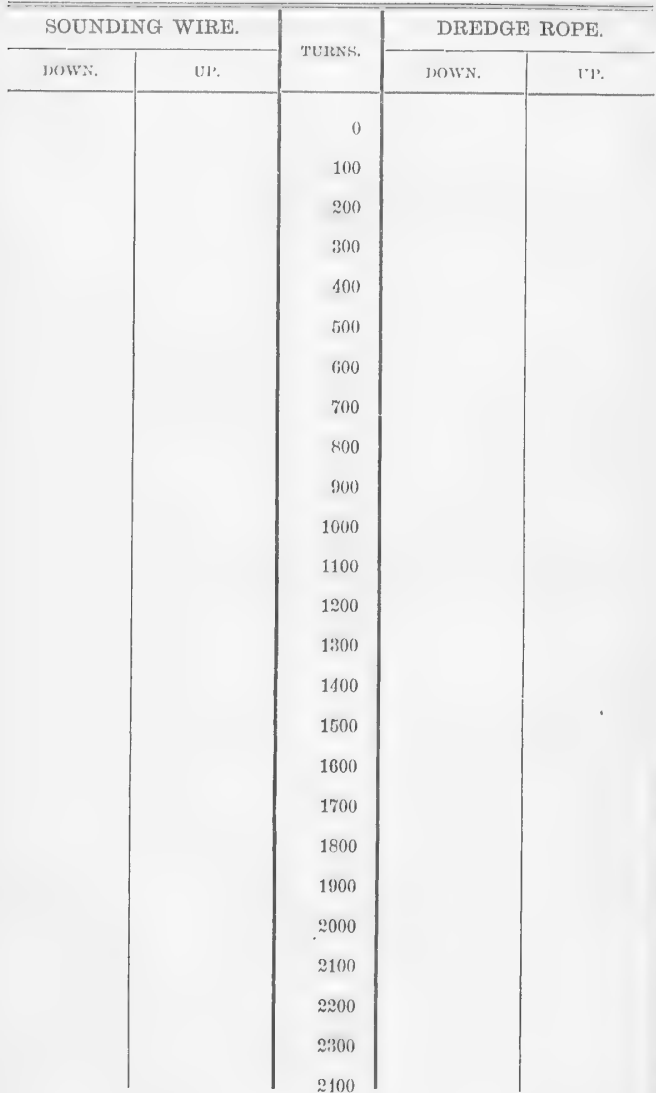




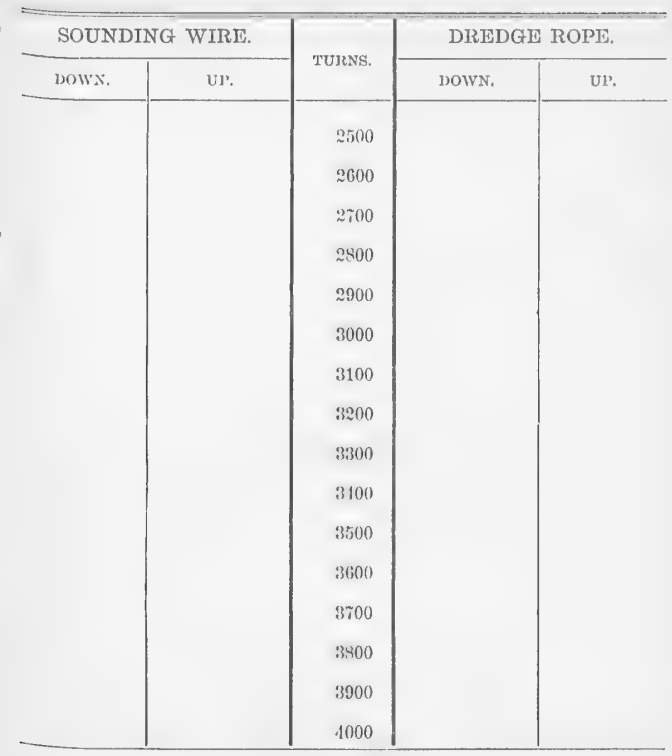

SERIAL TEMIPRATURES.

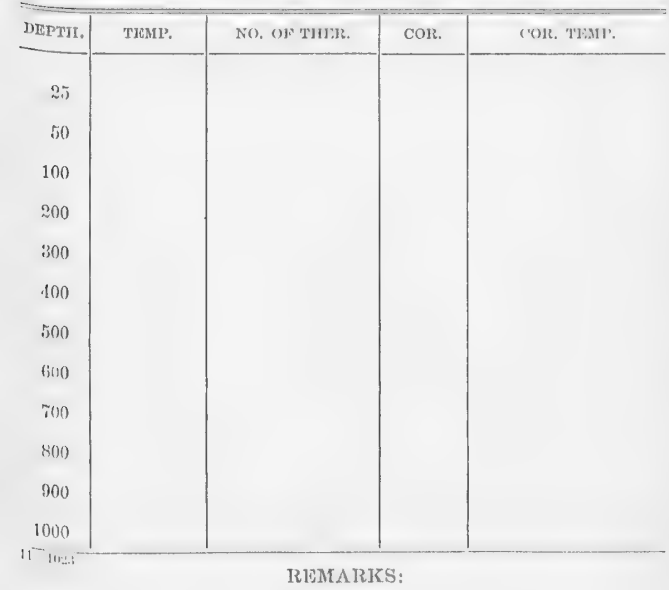


No.

Date

Machine, Reet.
Turns
Cor +
Depth

Shot or Zead

\section{Bottom}

Bottom temperature

No. of thermometer

Cor.

Corrected temperature

Air

Surface

Drift

Trawl or dredge

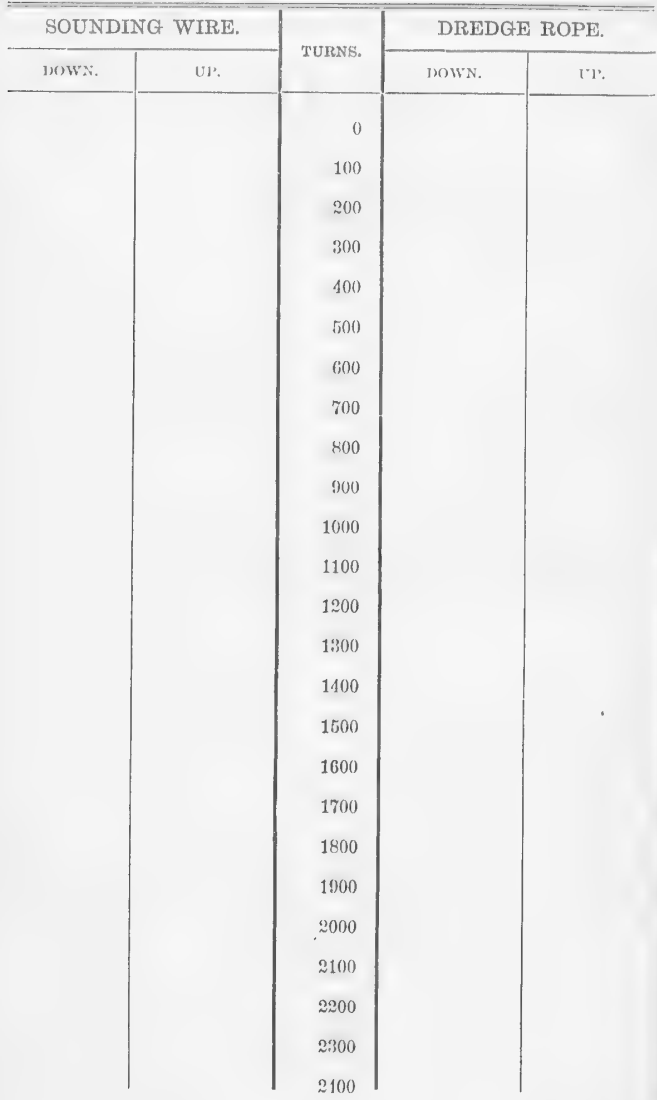


No.

Date

Machine. Recl.
Turns
Cor.t
Depth

Shot or lead

\section{Bottom}

Bottom temperature

No. of thermometer

Cor.

Corrected temperature

Air

Surface

Drift

Trawl or dredge

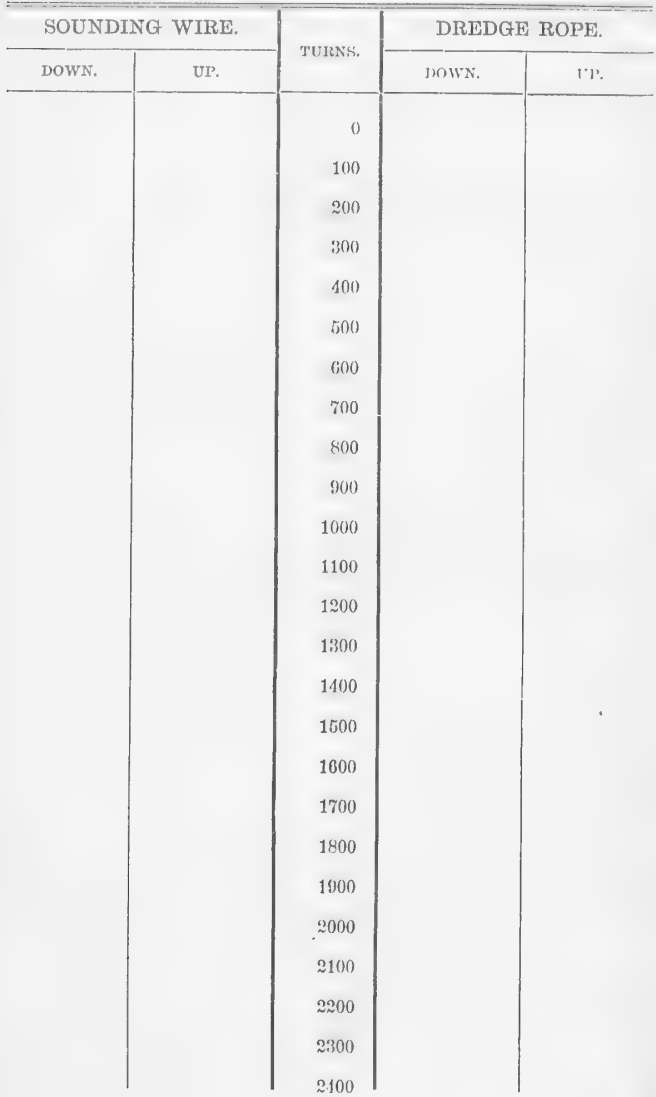




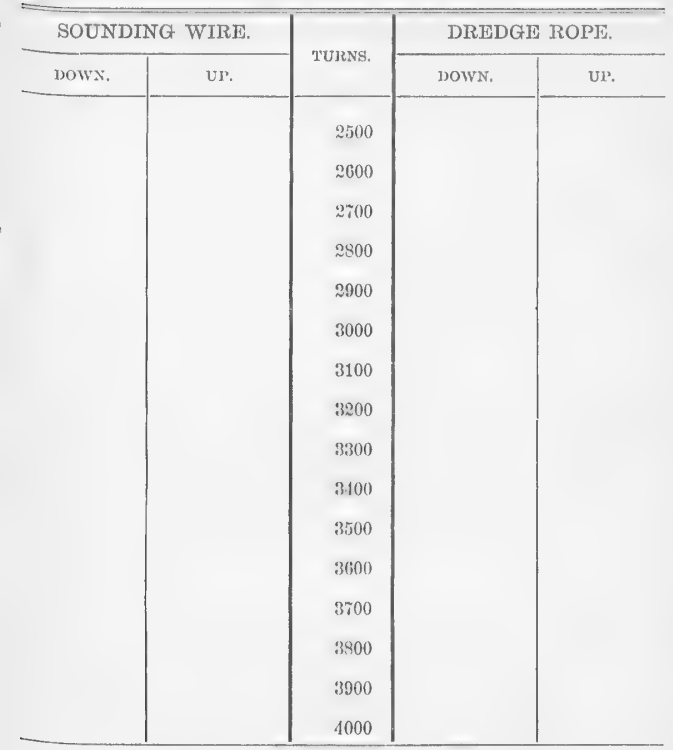

SERTAL TWMPERATURES.

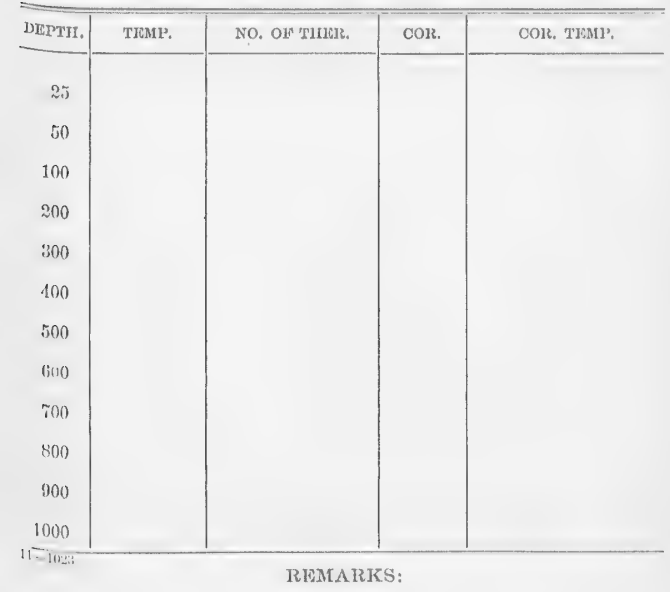


No.

\section{Date}

Machine. Reet.
Turns
Cor.t
Depth

Shot or lead

\section{Bottom}

\section{Bottom temperature}

No. of thermometer

Cor.

Corrected temperature
Air
Surface
Drift

Trawl or dredge

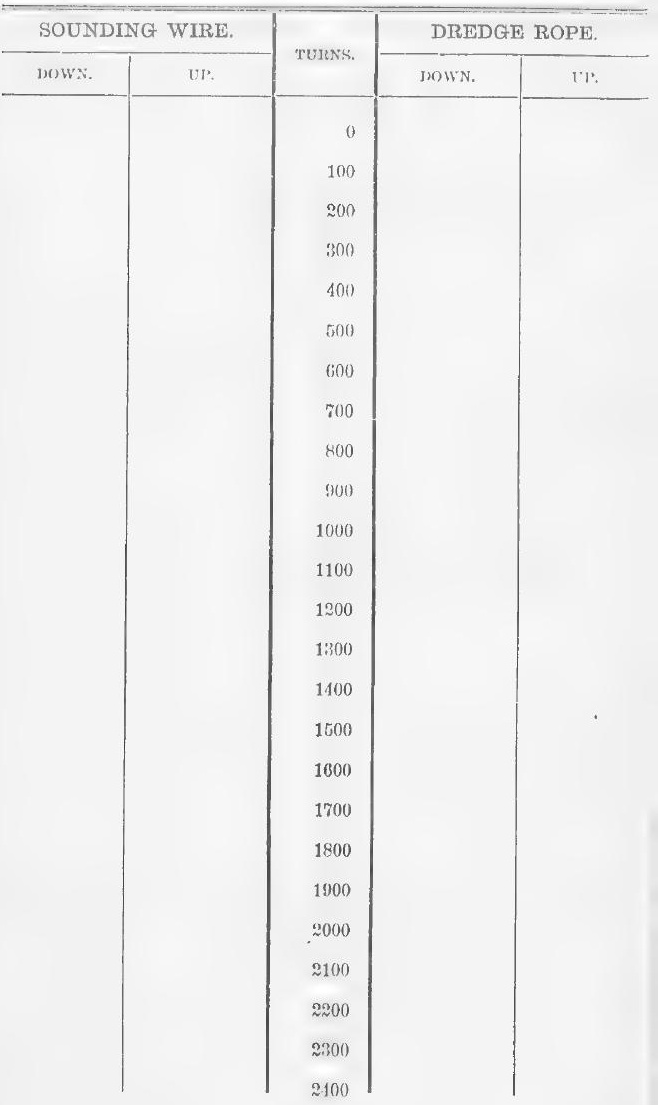


No.

Date

Machine. Reel.
Turns
Cor:.
Depth

Shot or lead

\section{Bottom}

\section{Bottom temperature}

No. of thermometer

Cor.

Corrected temperature
Air
Surface
Drift

Trawl or dredge

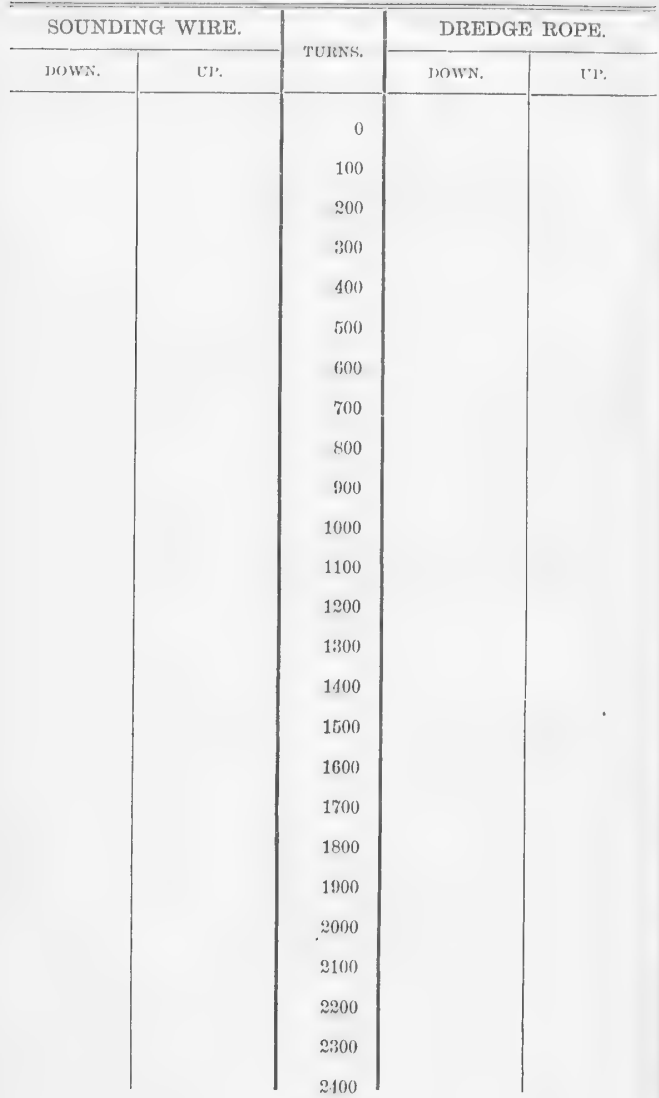


No.

Date

Mactine, Treel.
Turns
Cor. +
Depth

Shot or Zeart

\section{Bottom}

\section{Bottom temperature}

No. of thermometer

Cor.

Corrected temperature

Air

Surface

Drift

Trawl or dredge

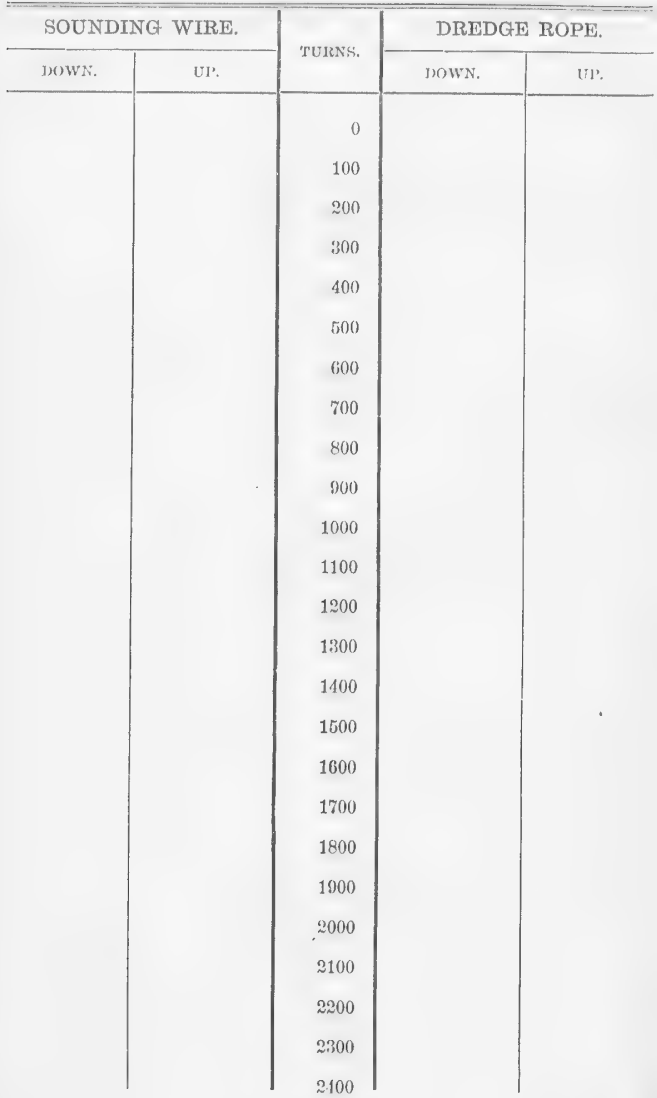


No.

Date

Machine. Reet.

Turns Cor.t Depth

Shot or lead

Bottom

Bottom temperature

No. of thermometer

Cor.

Corrected temperature

Air

Surface

Drift

Irawl or dredge

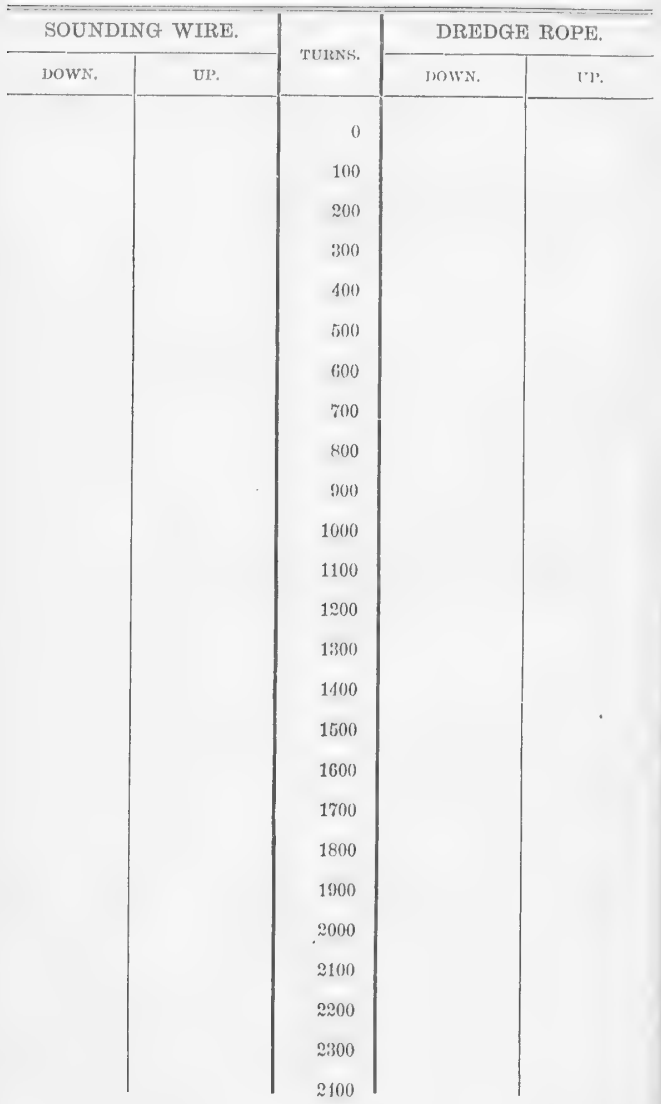




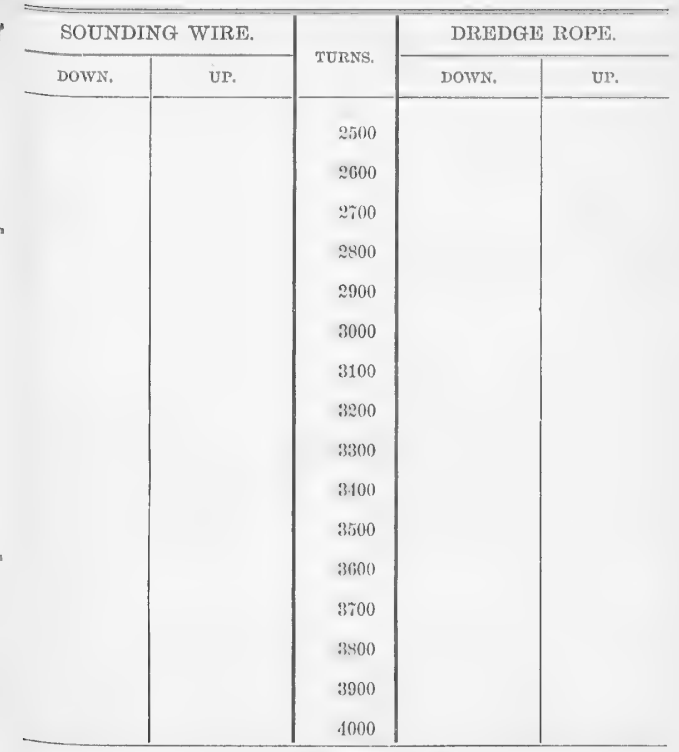

SERIAL TEMTPERATURES.

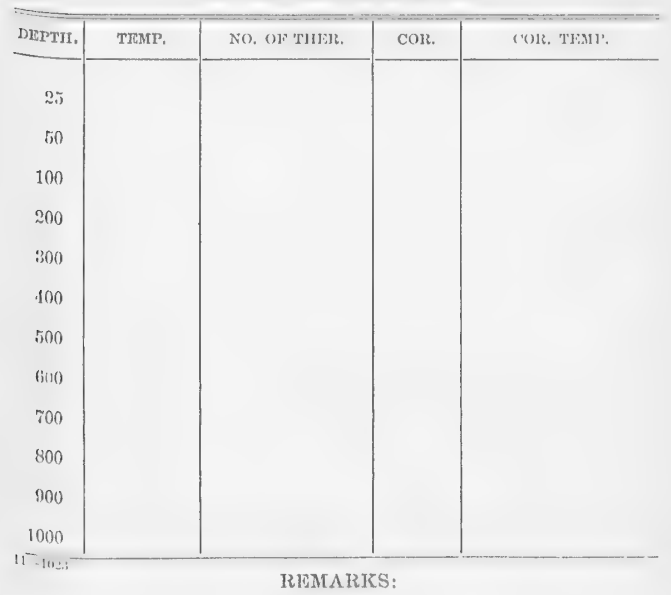


No.

Date

Machine. Reel.
Turns
Cor:+
Depth

Shot or lead

\section{Bottom}

\section{Bottom temperature}

No of thermometer

Cor.

Corrected temperature

Air

Surface

Drift

Trawl or dredge

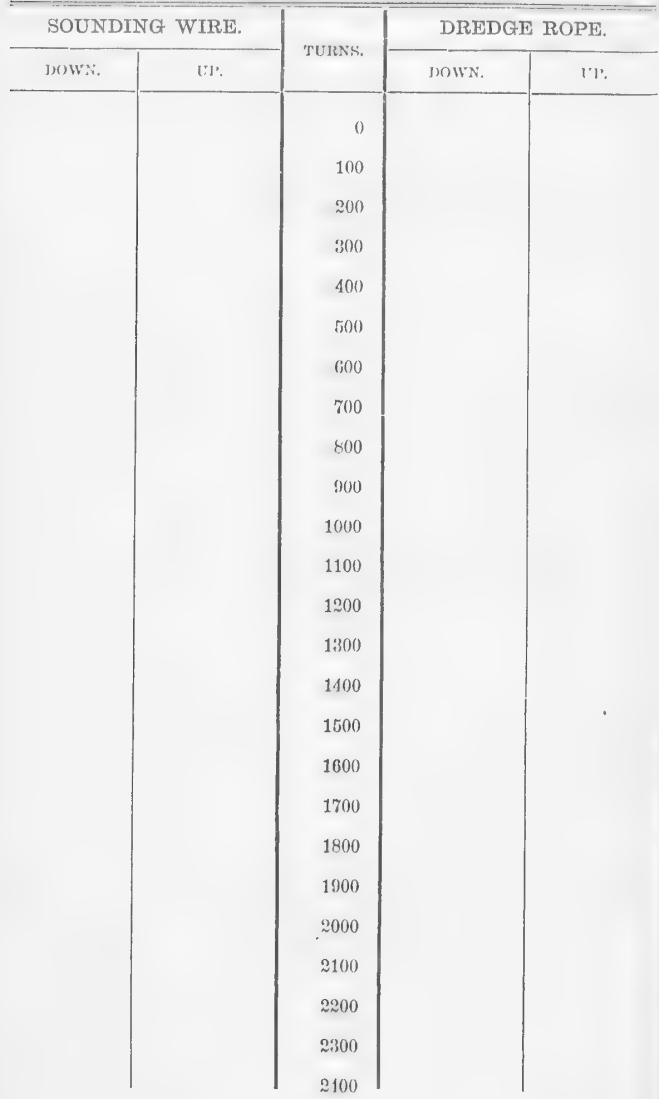




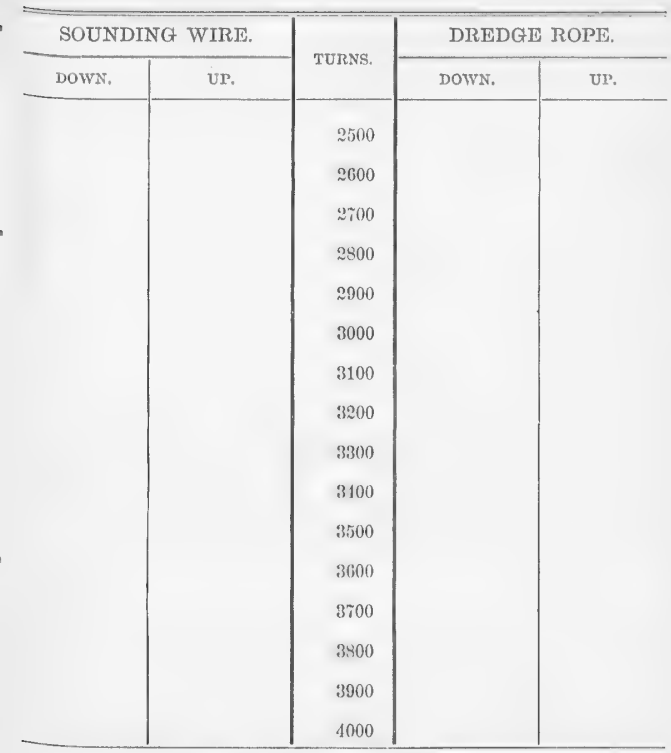

SERIAL TIEMPERATURES.

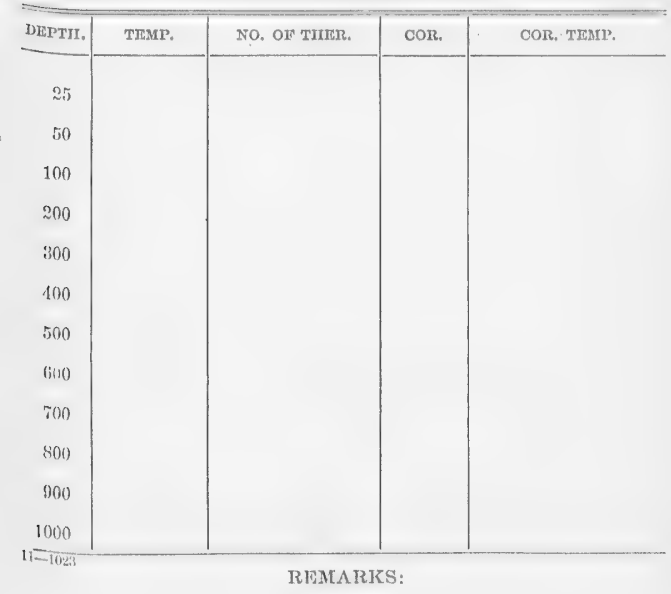


No.

Date

Machine. Reel.

Turns

Cors.

Depth

Shot or lead

\section{Bottom}

Bottom temperature

No of thermometer

Cor.

Corrected temperature

Air

Surface

Drift

Trawl or dredge

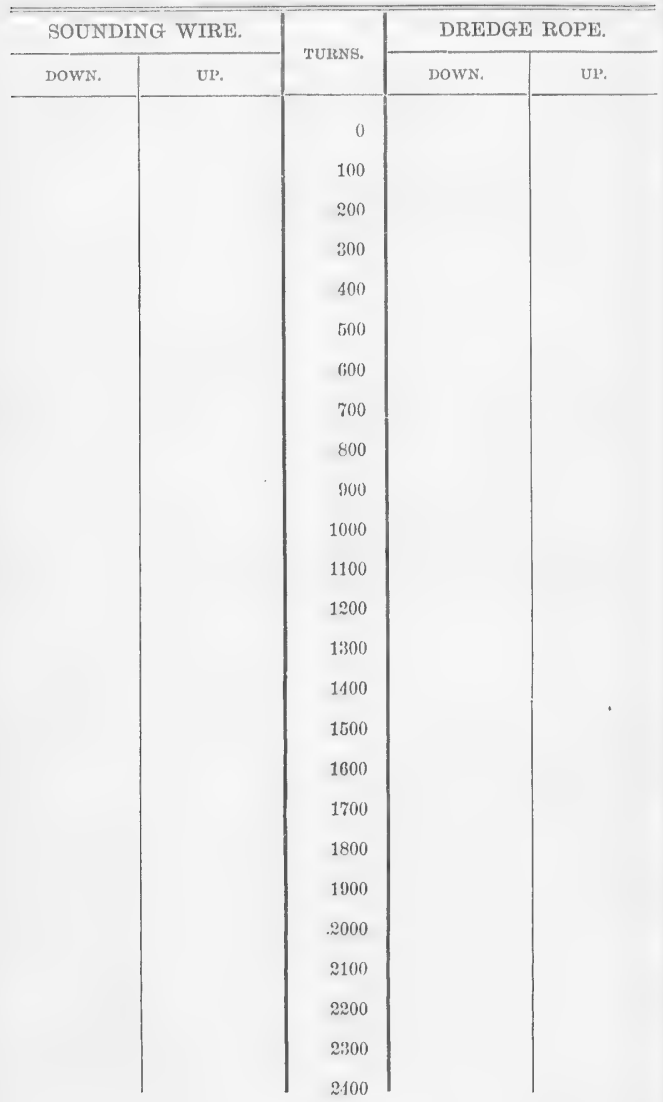




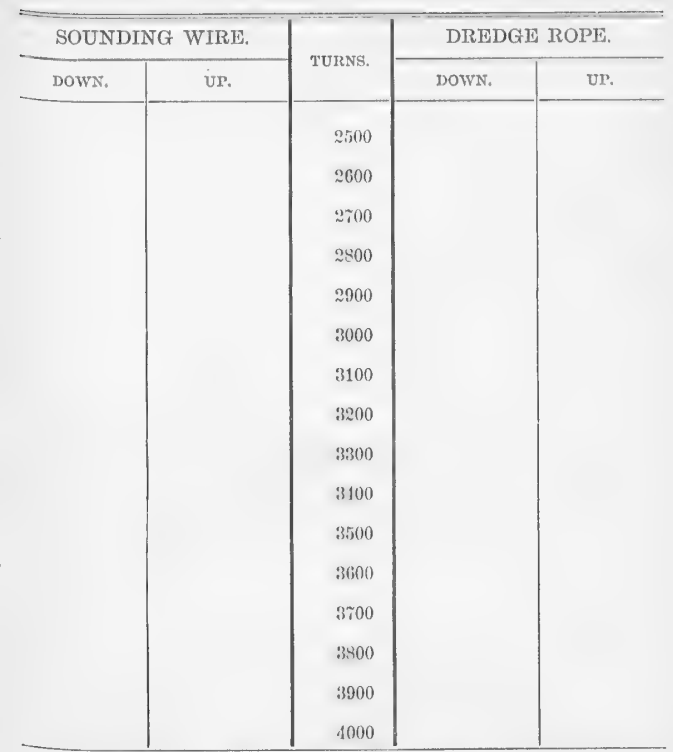

SERTAL, TEMPERATURES.

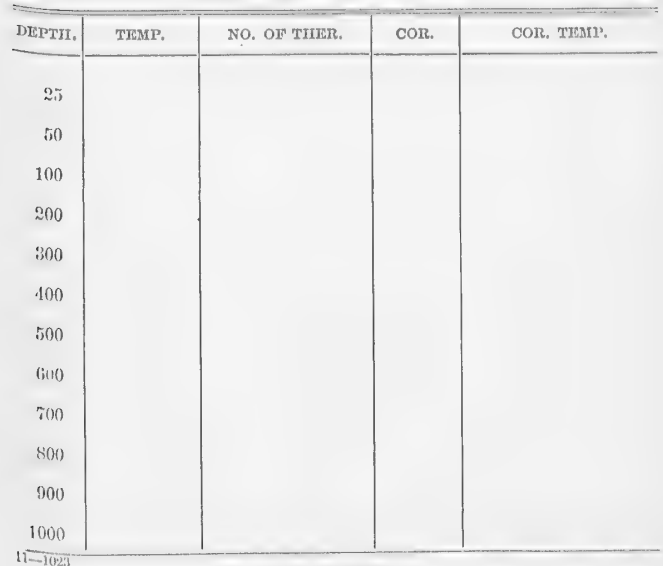

REMARKS: 
No.

Dote

Machine. Reel.

Turns

Cor, +

Depth

Shot or lead

Bottom

Bottom temperature

No. of thermometer

Cor.

Corrected temperature

Air

Surface

Drift

Trawl or dredge

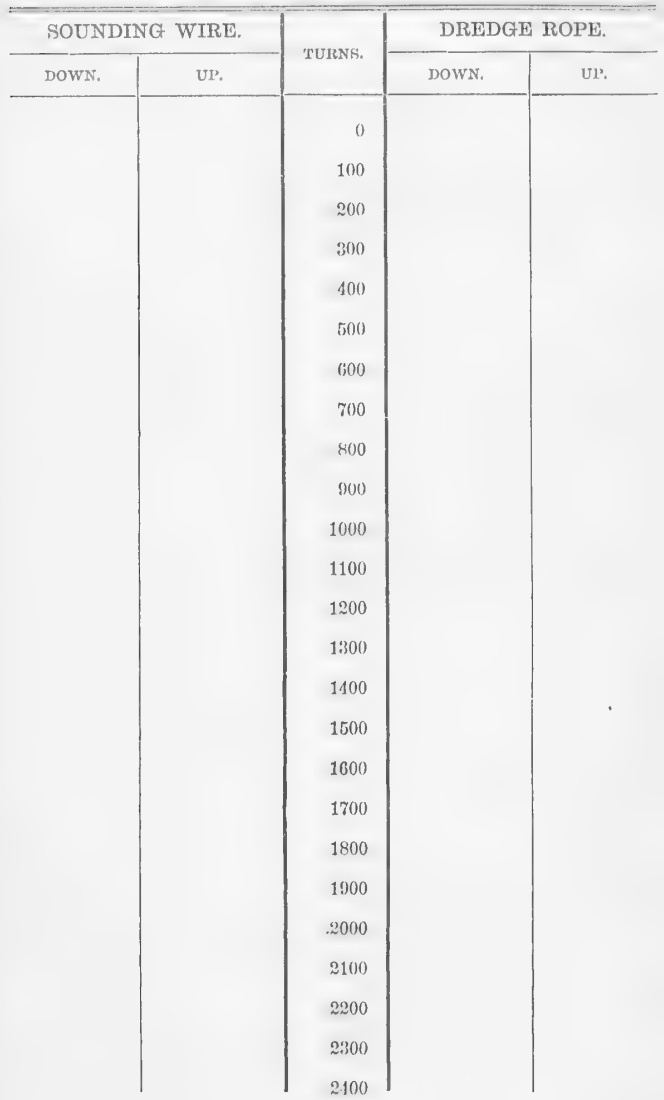


No. Date

Machine. Reel.

Turns

Cor.+

Depth

Shot or lead

Bottom

Bottom temperature

No. of thermometer

Cor.

Corrected temperature

Air

Surface

Drift

Trawt or dredge

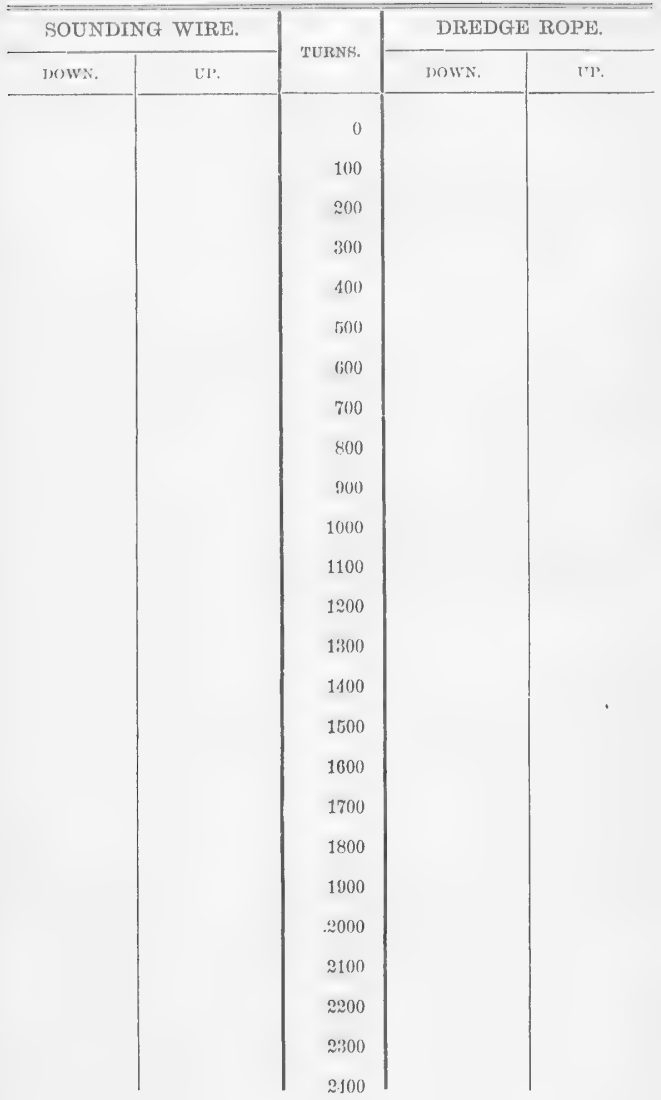


No.

Date

Machine. Reel.

Turns

Cori,

Depth

Shot or lead

J3ottom

Bottom temperature

No. of thermometer

Cor.

Corrected temperature

Air

Surface

Drift

Trawt or dredge

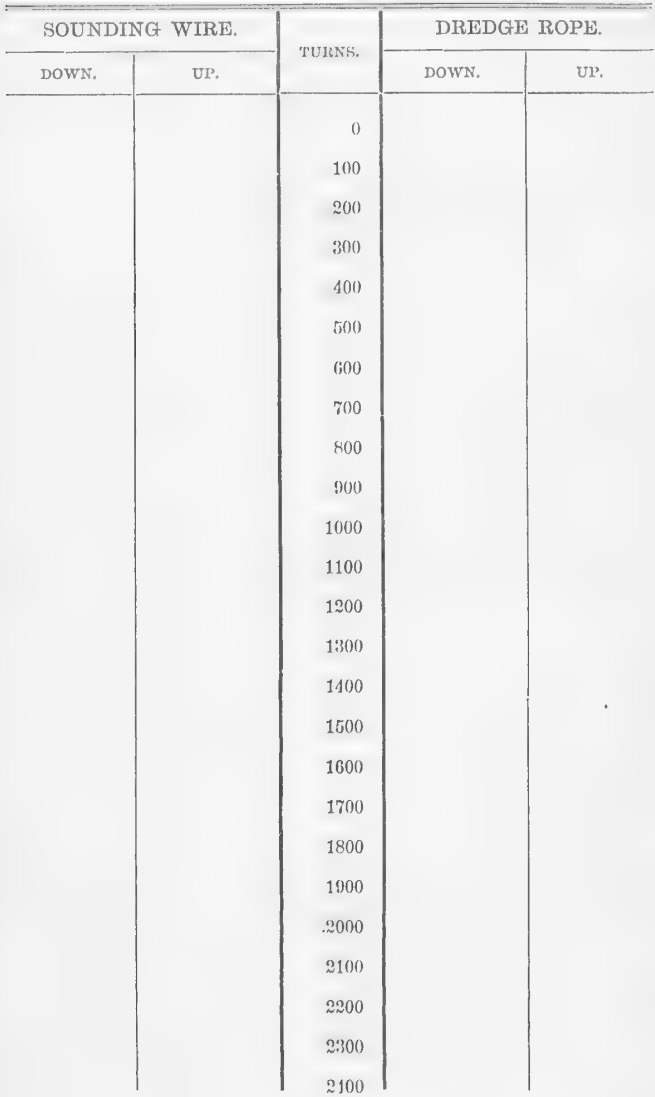




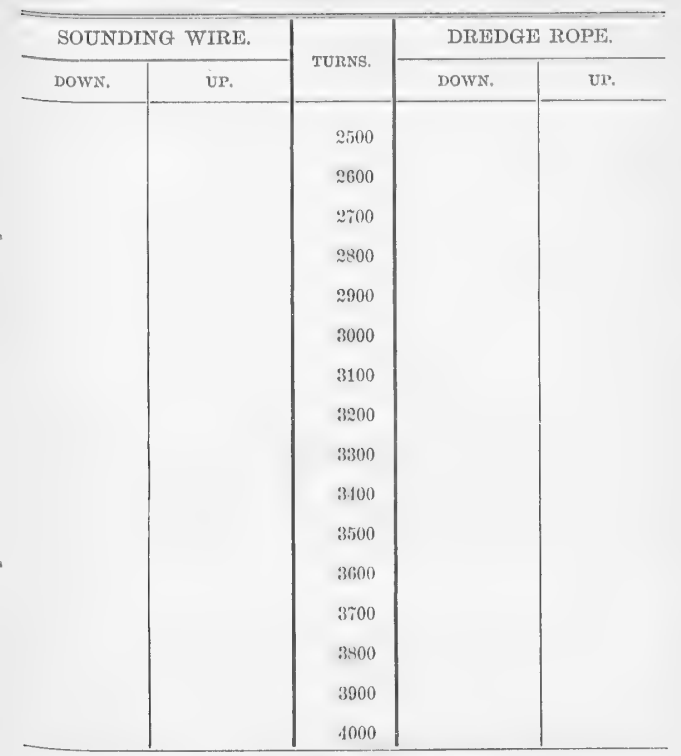

SERIAL TEMIPERATURES.

RFMARKS: 
No.

\section{Date}

Machine. Reel.

\section{Turns}

Cor, +

Depth

Shot or lead

\section{Bottom}

\section{Bottom temperature}

No. of thermometer

Cor.

Corrected temperature

Air

Surface

Drift

Trowl or dredge

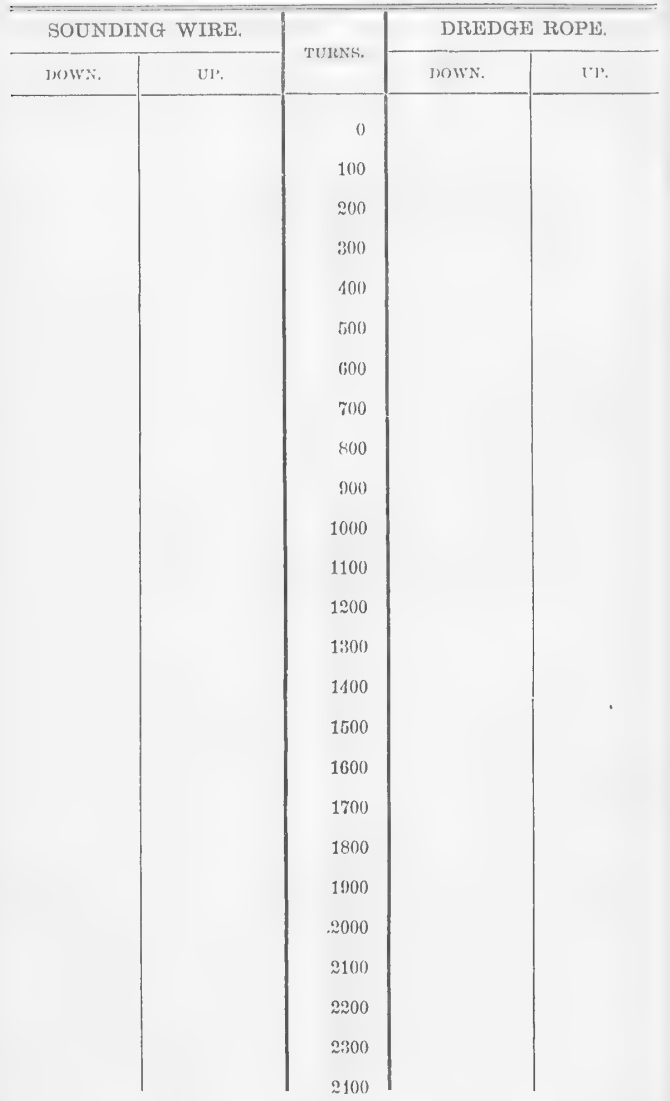




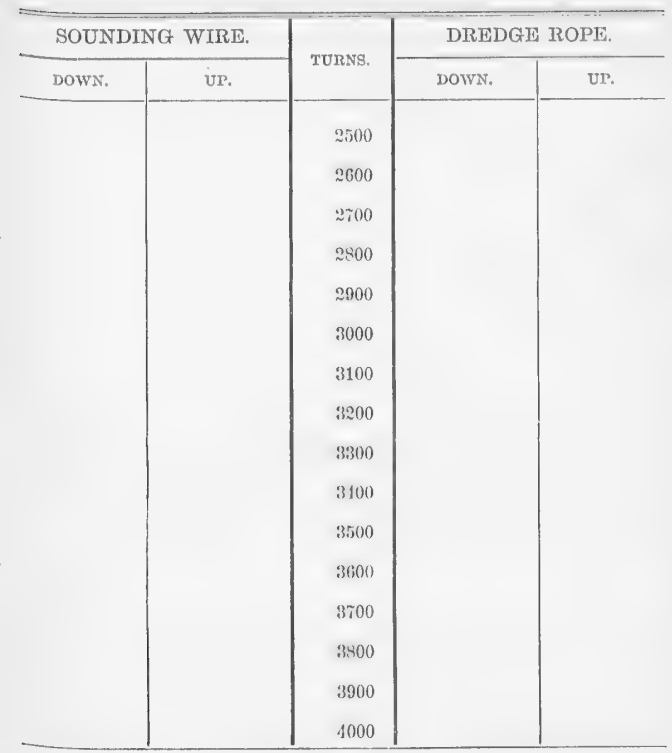

SERIAI TEMPERATUIRES.

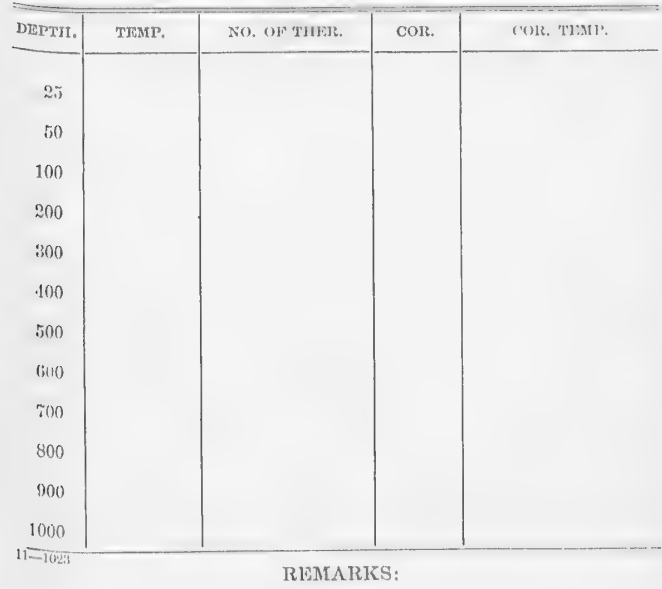


No.

Date

Machine, Reel.

\section{Turns}

Corr, +

Depth

Shot or lead

\section{Bottom}

\section{Bottom temperature}

No. of thermometer

Cor.

Corrected temperature

Air

Surface

Drift

Trawl or dredge

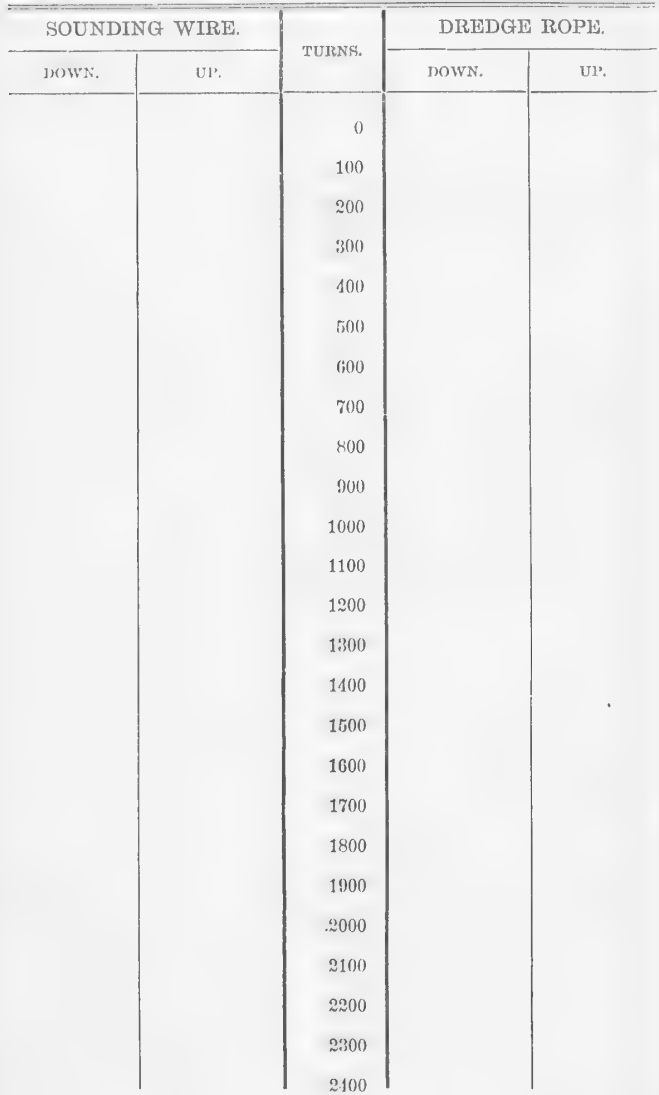


No.

Date

Machine, Reel.

Turns

Cor.t

Depth

Shot or lead

Bottom

Bottom temperature

No. of thermometer

Cor:

Corrected temperature

Air

Surface

Drift

Trawl or dredge

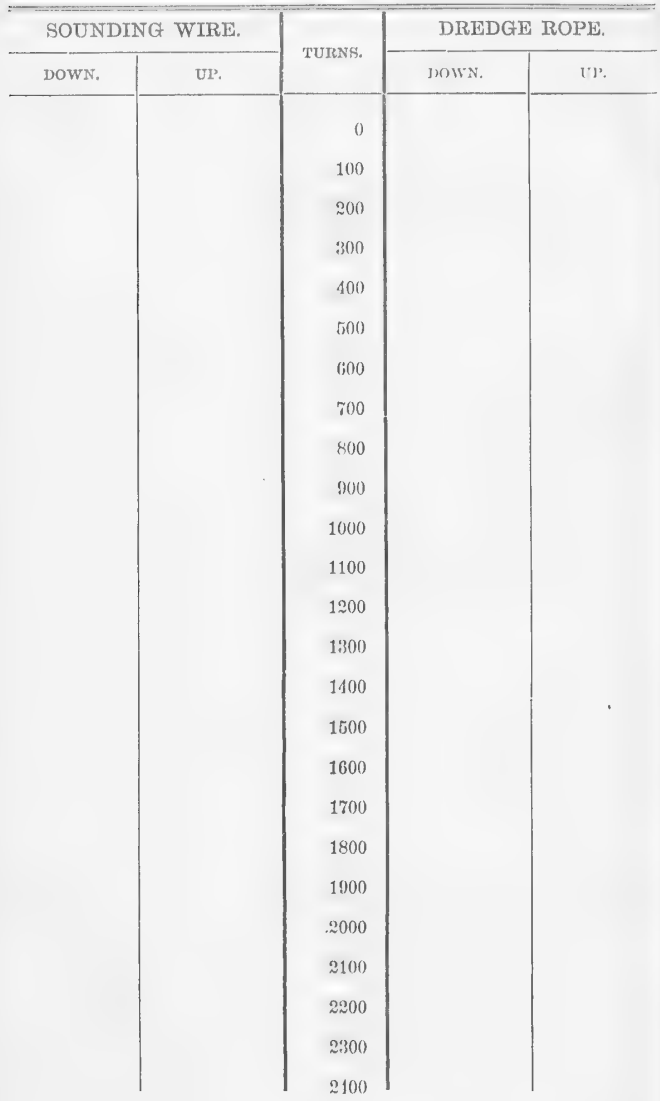


No.

Date

Machine, Reel.

Turns

Cor.t

Depth

Shot or lead

\section{Bottom}

Bottom temperature

No. of thermometer

Cor.

Corrected temperature

Air

Surface

Drift

Trawl or dredge

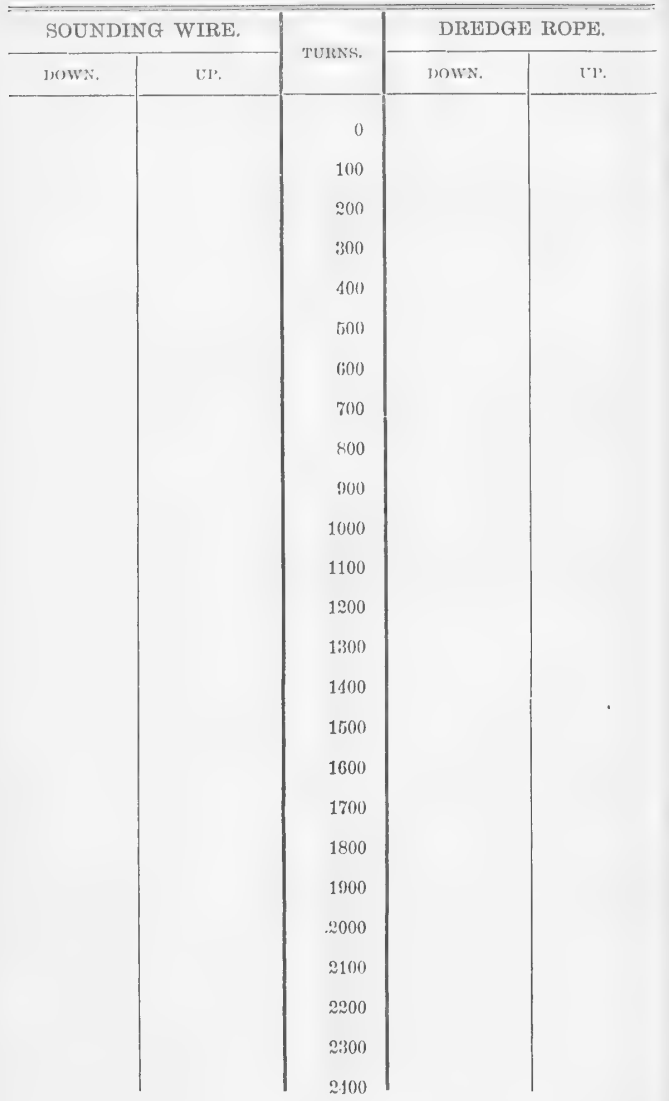


No.

Date

Machine. Reel.
Turns
Cor.t
Depth

Shot or lead

Bottom

Bottom temperature

No. of thermometer

Cor.

Corrected temperature

Air

Surface

Drift

Trawl or aredge

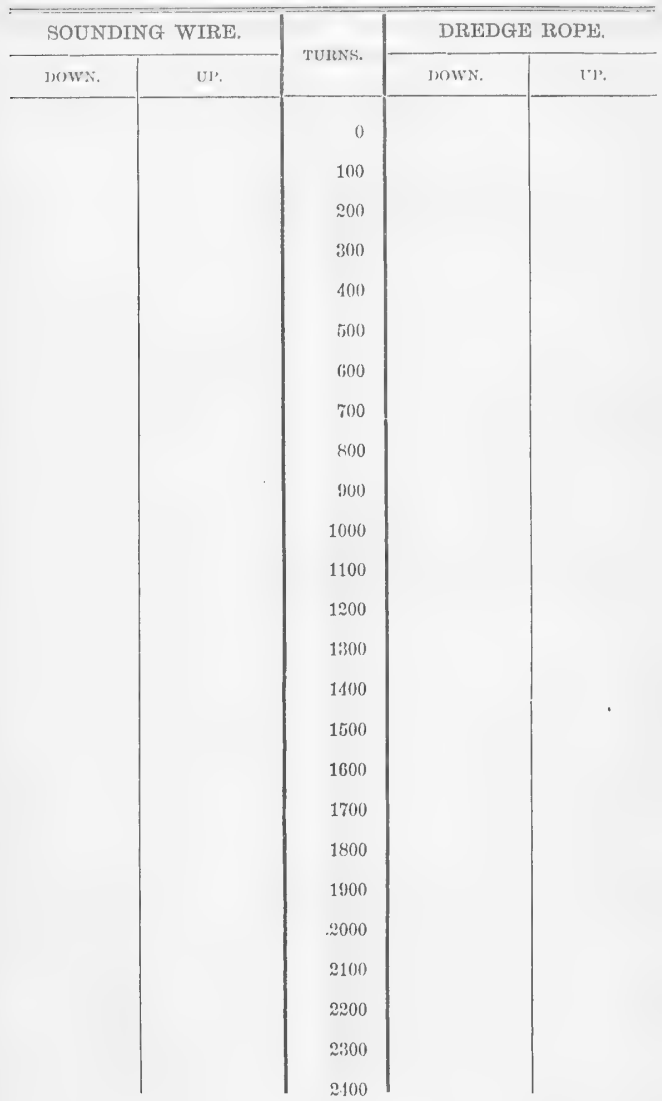




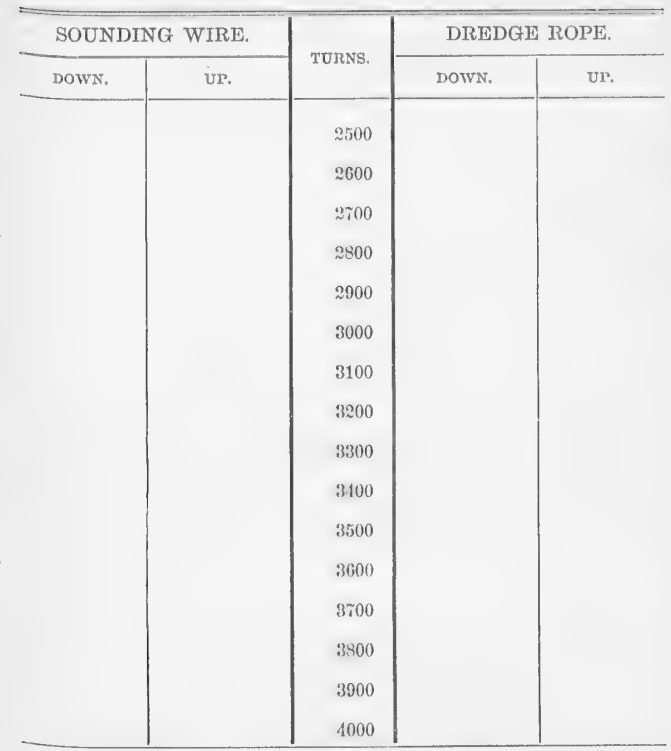

SERIAL TEMIPER $\Lambda$ TURES.

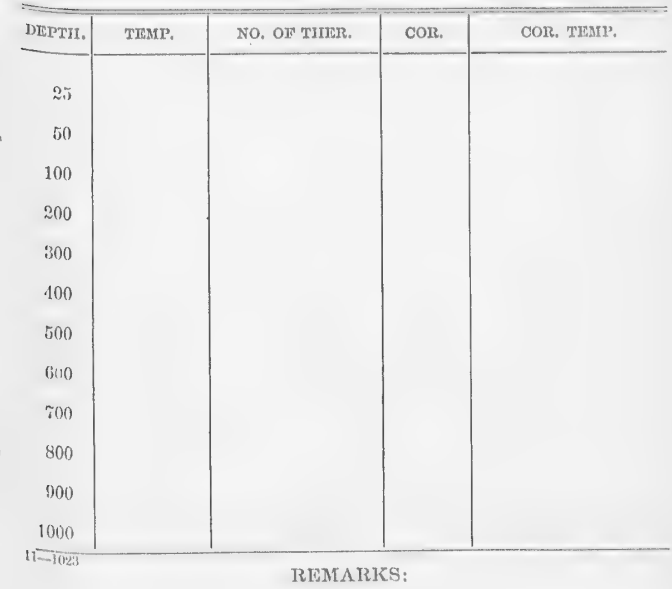


No.

Date

Machine, Reel.

Turns

Cor.t

Depth

Shot or lead

Bottom

Bottom temperature

No. of thermometer

Cor.

Corrected temperature

Air Surface Drift

Trawt or dredge

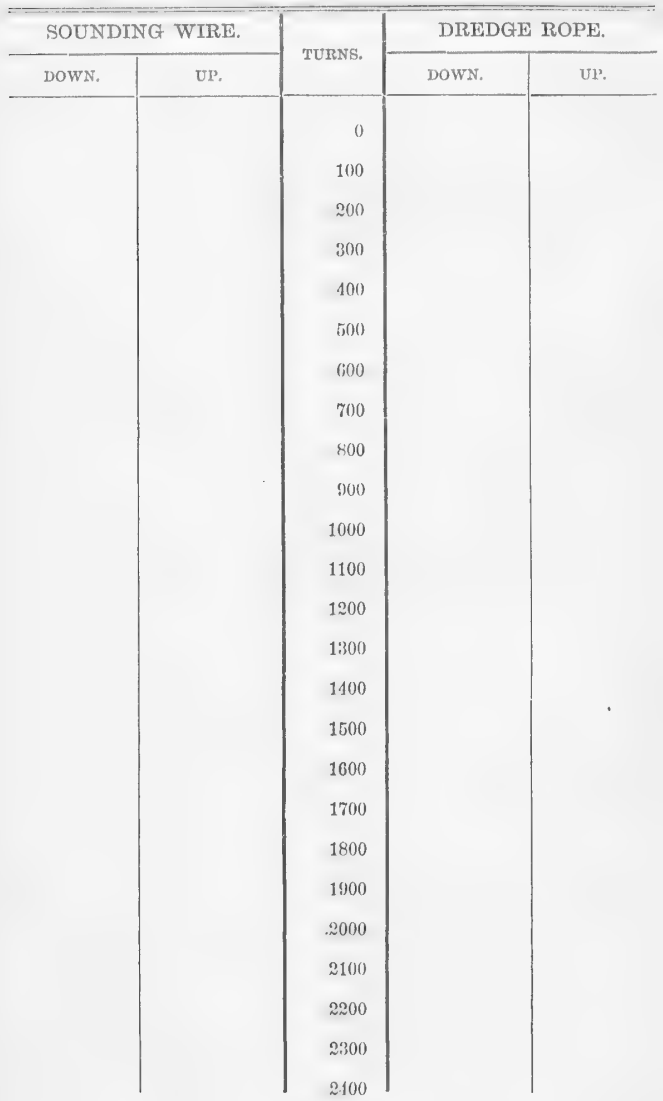




\begin{tabular}{|c|c|c|c|c|}
\hline \multicolumn{2}{|c|}{ SOUNDING WIRE. } & \multirow{2}{*}{ TURNS. } & \multicolumn{2}{|c|}{ DREDGE ROPE. } \\
\hline DOWN. & UP. & & DOWN. & Ur. \\
\hline & & 2500 & & \\
\hline & & 2600 & & \\
\hline & & 2700 & & \\
\hline & & 2800 & & \\
\hline & & 2900 & & \\
\hline & & 3000 & & \\
\hline & & 3100 & & \\
\hline & & 3200 & & \\
\hline & & 3300 & & \\
\hline & & 3400 & & \\
\hline & & 3500 & & \\
\hline & & $3600)$ & & \\
\hline & & 3700 & & \\
\hline & & 3800 & & \\
\hline & & 3900 & & \\
\hline & & 4000 & & \\
\hline
\end{tabular}

SERIAL TEMPERATURES.

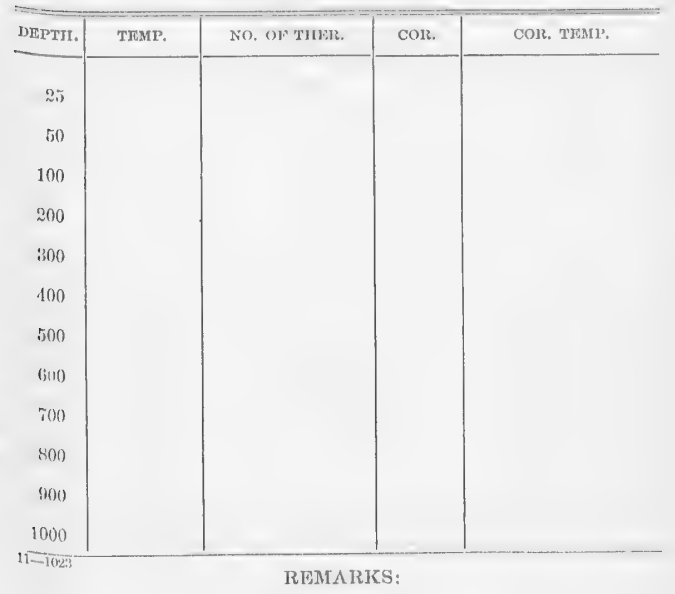


No.

Date

Machine, Reel.

Turns

Cor ,

Depth

Shot or lead

\section{Bottom}

\section{Bottom temperature}

No. of thermometer.

Cor.

Corrected temperature

Air

Surface

Drift

Trawt or dredge

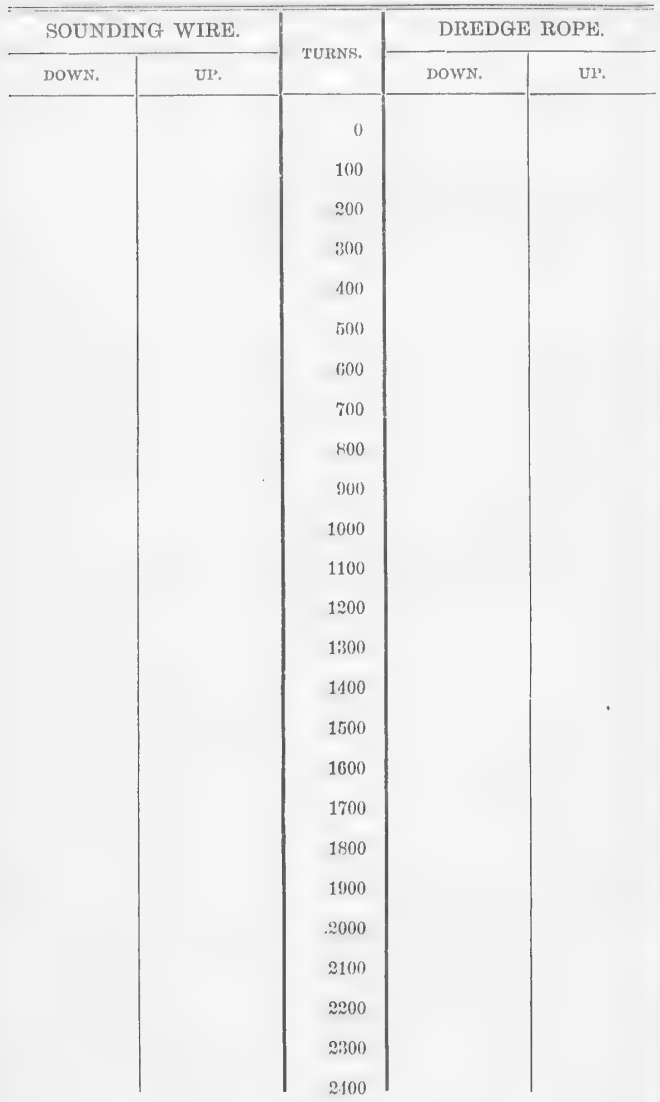




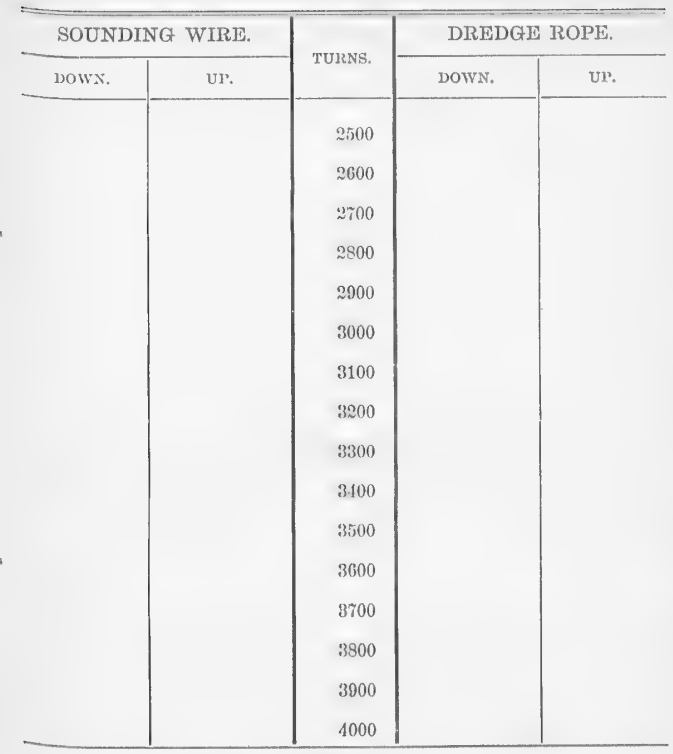

SERIAL TEMPERATURES.

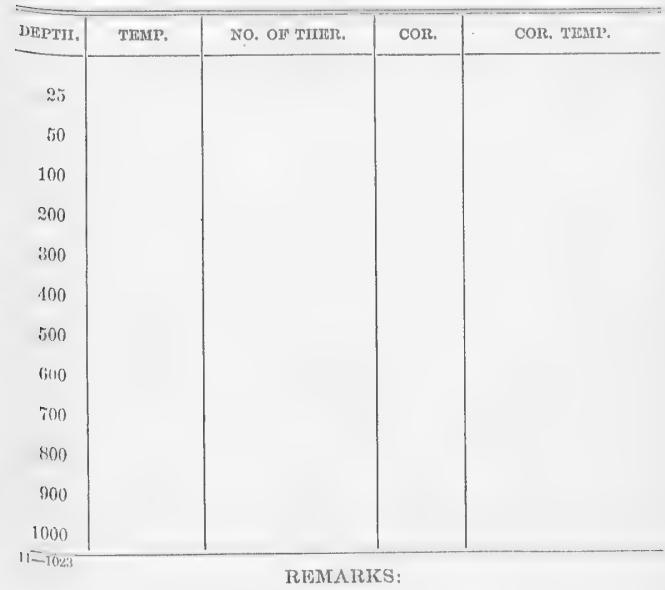


No.

Date

Machine. Rect.

Turns

Cor.t

Depth

Shot or lead

Bottom

Bottom temperature

No of thermometer.

Cor.

Corrected temperature

Air

Surface

Drift

Trawt or dredge

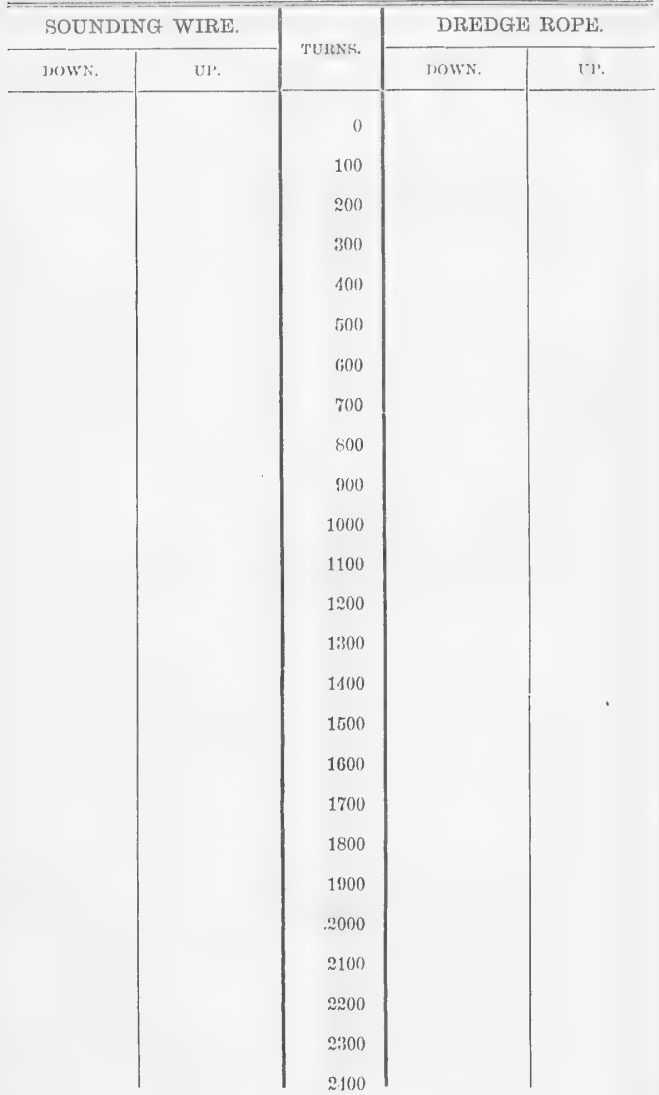


No.

Date

Machine. Reel.

Turns Cort+ Depth

Shot or lead

Bottom

Bottom temperature

No. of thermometer

Cor.

Corrected temperature

Air

Surface

Drift

Trawl or dredge

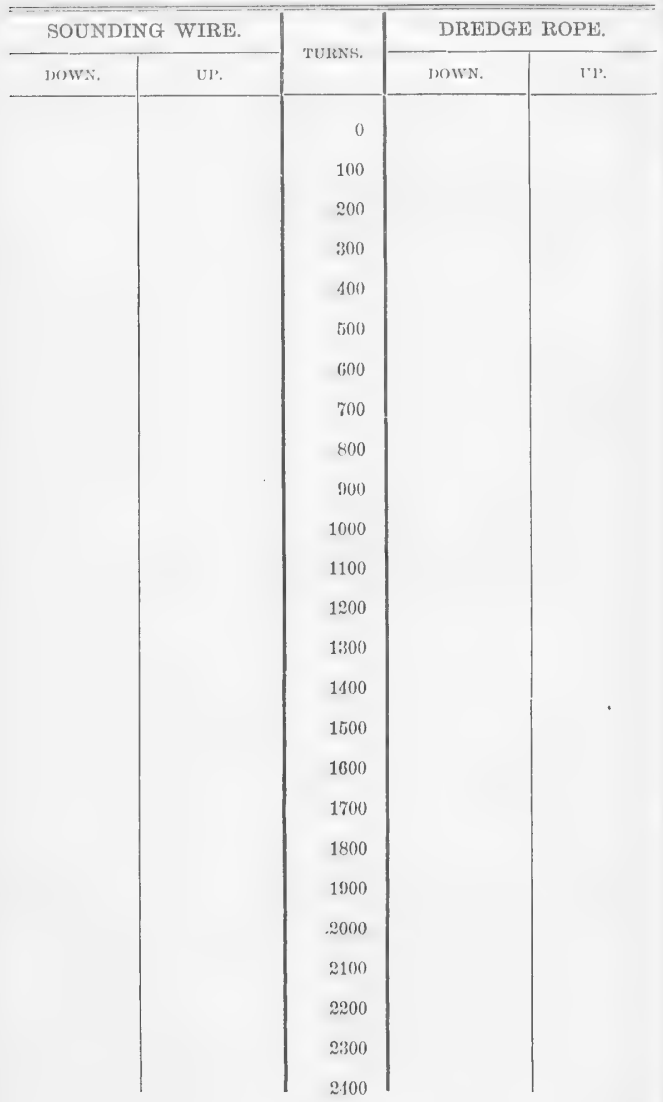




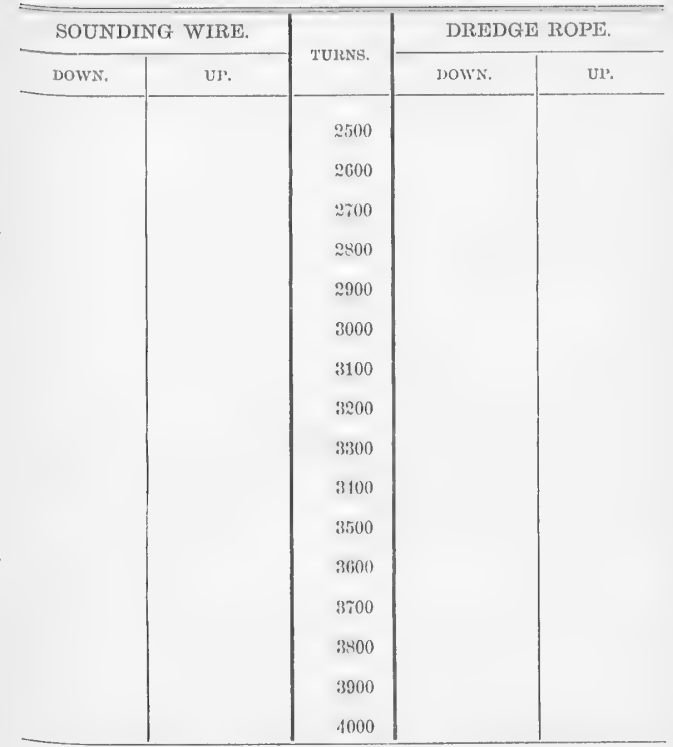

SEIRIAL THMPERATURES.

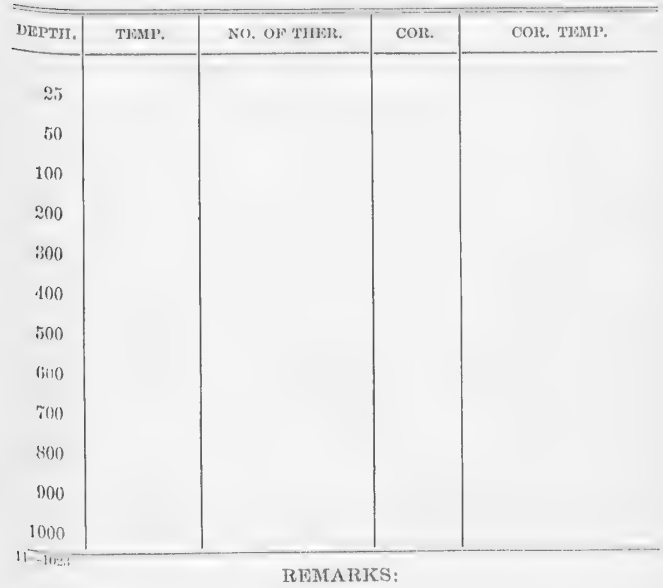


No.

Date

Machine. Reet.

Turns

Cor.t

Depth

Shot or lead.

Bottom

Bottom temperature

No. of thermometer.

Cor.

Corrected temperature

Air Surface Drift

Trawl or dredge

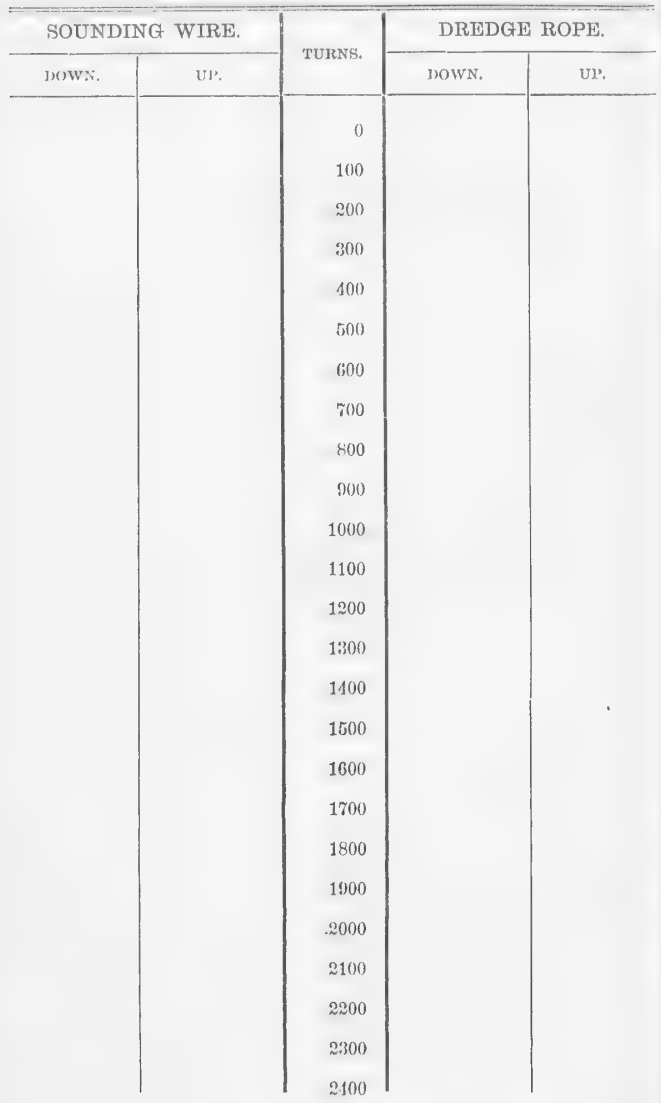




\begin{tabular}{|c|c|c|c|c|}
\hline \multicolumn{2}{|c|}{ SOUNDING WIRE. } & \multirow{2}{*}{ TURAS. } & \multicolumn{2}{|c|}{ DREDGE ROPE. } \\
\hline DOWN. & Us. & & JOWN. & U1. \\
\hline & & 2500 & & \\
\hline & & 2000 & & \\
\hline & & 2700 & & \\
\hline & & 2800 & & \\
\hline & & 2900 & & \\
\hline & & 3000 & & \\
\hline & & 3100 & & \\
\hline & & 3200 & & \\
\hline & & 3300 & & \\
\hline & & 3100 & & \\
\hline & & 3500 & & \\
\hline & & 3600 & & \\
\hline & & $3 \pi 00$ & & \\
\hline & & 3800 & & \\
\hline & & 3900 & & \\
\hline & & 4000 & & \\
\hline
\end{tabular}

SERIAL TEMPERATURES.

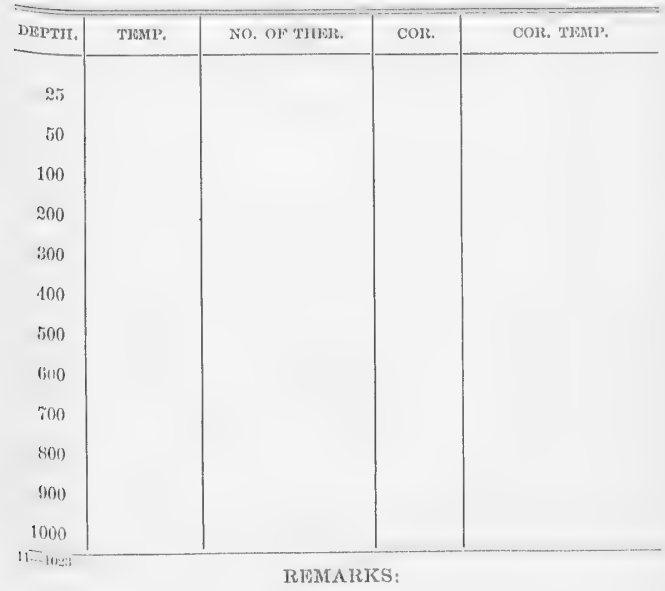


$\mathcal{N} o$.

\section{Date}

Machine. Reel.

Turns Cor.t Depth

Shot or lead

Bottom

Bottom temperature

No. of thermometer

Cor.

Corrected temperature

Air Surface Drift

Trawl or dredge

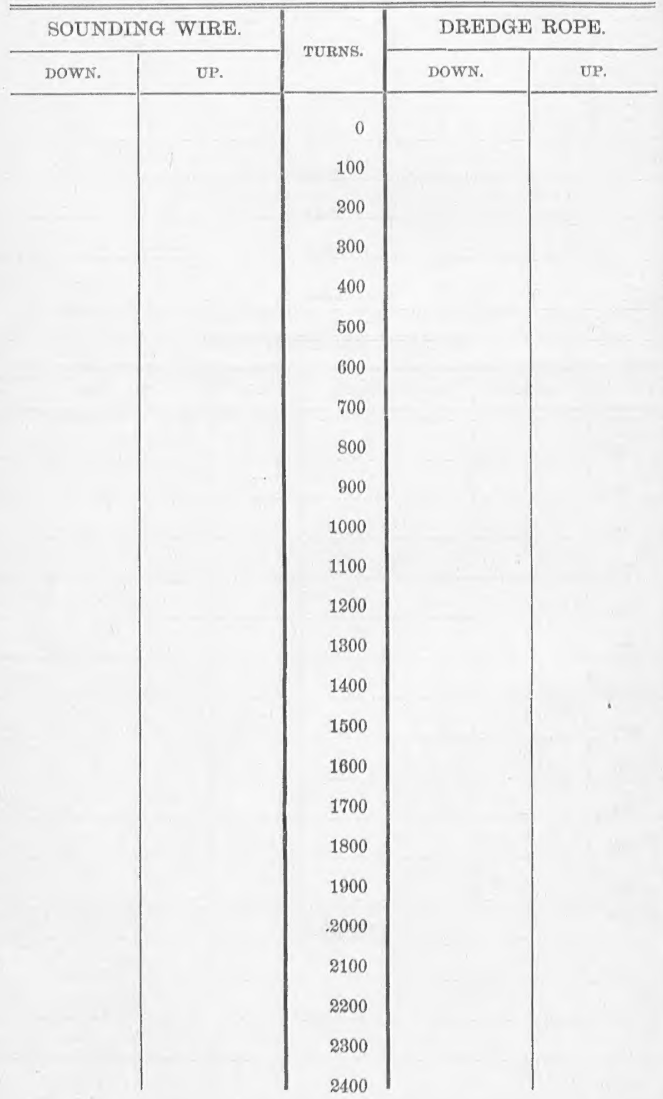




\begin{tabular}{|c|c|c|c|c|}
\hline \multicolumn{2}{|c|}{ SOUNDING WIRE. } & \multirow{2}{*}{ TURNS. } & \multicolumn{2}{|c|}{ DREDGE ROPE. } \\
\hline DOWN. & UP. & & Down. & UP. \\
\hline & & 2500 & & \\
\hline & & 2600 & & \\
\hline & & 2700 & & \\
\hline & & 2800 & & \\
\hline & & 2900 & & \\
\hline & & 3000 & & \\
\hline & & 3100 & & \\
\hline & & 3200 & & \\
\hline & & 3300 & & \\
\hline & & 3400 & & \\
\hline & & 3500 & & \\
\hline & & 3600 & & \\
\hline & & 8700 & & \\
\hline & & 3800 & & \\
\hline & & 3900 & & \\
\hline & & 4000 & & \\
\hline
\end{tabular}

SERIAL TEMPERATURES.

\begin{tabular}{r|r|r|r|r}
\hline DEPTH. & TEMP. & NO. OF THER. & COR. & COR. TEMP. \\
\hline 25 & & & & \\
50 & & & \\
100 & & & \\
200 & & & \\
300 & & & \\
400 & & & \\
500 & & & \\
600 & & & \\
700 & & & \\
800 & & & \\
900 & & & \\
1000 & & & \\
$11=1023$ & & & \\
\hline
\end{tabular}




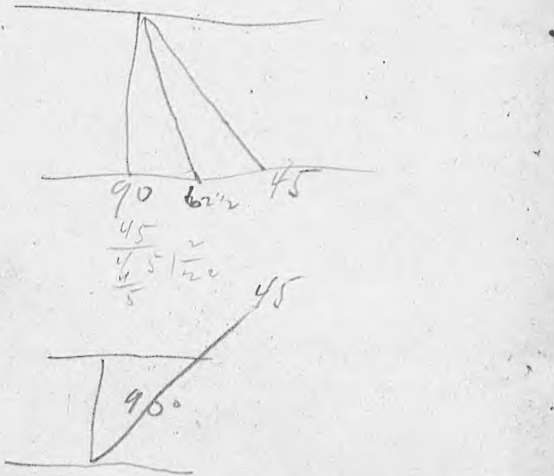

$2-31 \varepsilon$

$$
\begin{aligned}
& \frac{24^{22}}{3: 11}
\end{aligned}
$$

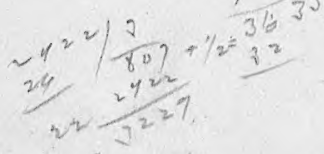

$$
\begin{aligned}
& r 10=\int_{\text {mos }} 3 \%
\end{aligned}
$$

